\title{
Stationary Black Holes: Uniqueness and Beyond
}

\author{
Piotr T. Chruściel \\ University of Vienna \\ email: piotr.chrusciel@univie.ac.at \\ http://homepage.univie.ac.at/piotr.chrusciel \\ João Lopes Costa \\ Instituto Universitário de Lisboa (ISCTE-IUL), Lisboa, Portugal \\ Centro de Análise Matemática, Geometria e Sistemas Dinâmicos, \\ Instituto Superior Técnico, Universidade Técnica de Lisboa, Portugal \\ email: jlca@iscte.pt \\ Markus Heusler \\ ITP, University of Zurich, CH-8057 Zurich \\ (at the time of writing original 1998 version) \\ email: markus.heusler@rsnag.ch
}

Accepted on 29 March 2012

Published on 29 May 2012

\begin{abstract}
The spectrum of known black-hole solutions to the stationary Einstein equations has been steadily increasing, sometimes in unexpected ways. In particular, it has turned out that not all black-hole-equilibrium configurations are characterized by their mass, angular momentum and global charges. Moreover, the high degree of symmetry displayed by vacuum and electrovacuum black-hole spacetimes ceases to exist in self-gravitating non-linear field theories. This text aims to review some developments in the subject and to discuss them in light of the uniqueness theorem for the Einstein-Maxwell system.
\end{abstract}

This review is licensed under a Creative Commons Attribution-Non-Commercial-NoDerivs 3.0 Germany License. http://creativecommons .org/licenses/by-nc-nd/3.0/de/ 


\section{Imprint / Terms of Use}

Living Reviews in Relativity is a peer reviewed open access journal published by the Max Planck Institute for Gravitational Physics, Am Mühlenberg 1, 14476 Potsdam, Germany. ISSN 1433-8351.

This review is licensed under a Creative Commons Attribution-Non-Commercial-NoDerivs 3.0 Germany License: http://creativecommons.org/licenses/by-nc-nd/3.0/de/. Figures that have been previously published elsewhere may not be reproduced without consent of the original copyright holders.

Because a Living Reviews article can evolve over time, we recommend to cite the article as follows:

Piotr T. Chruściel, João Lopes Costa and Markus Heusler, "Stationary Black Holes: Uniqueness and Beyond",

Living Rev. Relativity, 15, (2012), 7. [Online Article]: cited [<date $>$ ], http://www.livingreviews.org/lrr-2012-7

The date given as $<$ date $>$ then uniquely identifies the version of the article you are referring to. 


\section{Article Revisions}

Living Reviews supports two ways of keeping its articles up-to-date:

Fast-track revision A fast-track revision provides the author with the opportunity to add short notices of current research results, trends and developments, or important publications to the article. A fast-track revision is refereed by the responsible subject editor. If an article has undergone a fast-track revision, a summary of changes will be listed here.

Major update A major update will include substantial changes and additions and is subject to full external refereeing. It is published with a new publication number.

For detailed documentation of an article's evolution, please refer to the history document of the article's online version at http://www. livingreviews.org/lrr-2012-7.

29 May 2012: Major update of the original version by Markus Heusler from 1998. Piotr T. Chruściel and João Lopes Costa succeeded to this review's authorship. Significantly restructured and updated all sections; changes are too numerous to be usefully described here. The number of references increased from 186 to 329. 


\section{Contents}

1 Introduction $\quad \mathbf{7}$

1.1 General remarks ......................... . . . 7

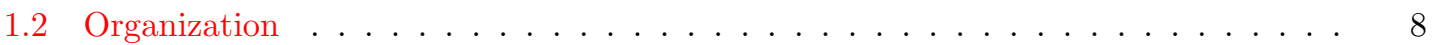

2 Definitions $\quad 9$

2.1 Asymptotic flatness . . . . . . . . . . . . . . . . . . . . . . . 9

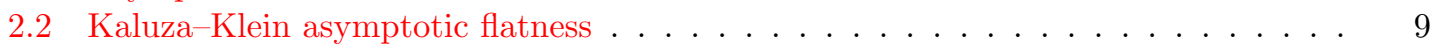

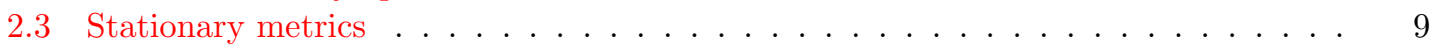

2.4 Domains of outer communications, event horizons . . . . . . . . . . . . . . . . . 10

2.5 Killing horizons . . . . . . . . . . . . . . . . . . . . . 11

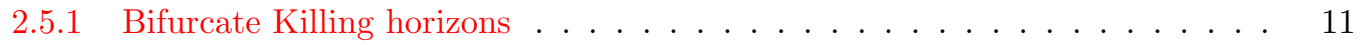

2.5.2 Killing prehorizons . . . . . . . . . . . . . . . . . 12

2.5.3 Surface gravity: degenerate, non-degenerate and mean-non-degenerate horizons 12

$2.6 I^{+}$-regularity . . . . . . . . . . . . . . . . . . . . 15

3 Towards a classification of stationary electrovacuum black hole spacetimes 16

3.1 Static solutions . . . . . . . . . . . . . . . . . . . . . 16

3.2 Stationary-axisymmetric solutions . . . . . . . . . . . . . . . . . . . . . . . . . . . . . . . . 17

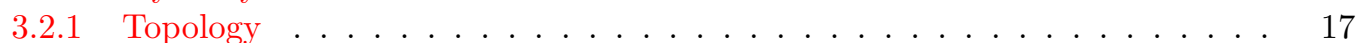

3.2 .2 Candidate metrics . . . . . . . . . . . . . . . . . 17

3.2 .3 The reduction . . . . . . . . . . . . . . . . . 18

3.2.4 The Robinson-Mazur proof . . . . . . . . . . . . . . . . . 19

3.2.5 The Bunting-Weinstein harmonic-map argument . . . . . . . . . . . . 19

3.2.6 The Varzugin-Neugebauer-Meinel argument . . . . . . . . . . . . . . . . 19

3.2.7 The axisymmetric uniqueness theorem . . . . . . . . . . . . . . 19

3.3 The no-hair theorem . . . . . . . . . . . . . . . . . . . . . 20

3.3.1 The rigidity theorem . . . . . . . . . . . . . . . . . . 20

3.3.2 The uniqueness theorem . . . . . . . . . . . . . . . 20

3.3.3 A uniqueness theorem for near-Kerrian smooth vacuum stationary spacetimes 22

3.4 Summary of open problems . . . . . . . . . . . . . . . . . . . . . . . . . . . . . . . . . . . . . . .

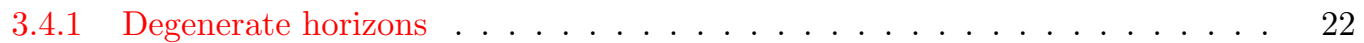

3.4.2 Rigidity without analyticity . . . . . . . . . . . . . . . . . . 24

3.4 .3 Many components? . . . . . . . . . . . . . . . . . . . 24

4 Classification of stationary toroidal Kaluza-Klein black holes 26

4.1 Black holes in higher dimensions . . . . . . . . . . . . . . . . . . . . . 26

4.2 Stationary toroidal Kaluza-Klein black holes . . . . . . . . . . . . . . . . . . 27

4.3 Topology of the event horizon . . . . . . . . . . . . . . . . . . . . . 27

4.4 Orbit space structure . . . . . . . . . . . . . . . . . . . . . . 28

4.5 KK topological censorship . . . . . . . . . . . . . . . . . . . . . . . . . . . . . . . . . . . . . . .

4.6 Classification theorems for $K K$-black holes . . . . . . . . . . . . . . . . . . . . . . 28

5 Beyond Einstein-Maxwell $\quad 31$

5.1 Spherically symmetric black holes with hair . . . . . . . . . . . . . . . . . . . . . . . . . 31

5.2 Static black holes without spherical symmetry . . . . . . . . . . . . . . . . . . . . . . . . . . . . . . .

5.3 The Birkhoff theorem . . . . . . . . . . . . . . . . . . . . . . . . . . . . . . . . . . . . 32

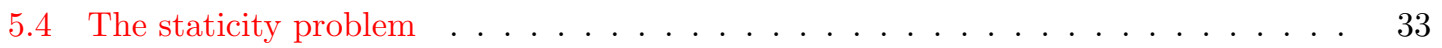

5.5 Rotating black holes with hair . . . . . . . . . . . . . . . . 33 
6 Stationary Spacetimes $\quad 35$

6.1 Reduction of the Einstein-Hilbert action . . . . . . . . . . . . . . . . 35

6.2 The coset structure of vacuum gravity . . . . . . . . . . . . . . . . . . . . . . . . . . . . . . . . . . . . . . .

6.3 Stationary gauge fields . . . . . . . . . . . . . . . . . . . . . . . . . . . . . . . . . . . . .

6.4 The stationary Einstein-Maxwell system . . . . . . . . . . . . . . . . 39

7 Some Applications $\quad 41$

7.1 The Mazur identity . . . . . . . . . . . . . . . . . . . . . . . . . . . . . . . . . . . . . . . . . . .

7.2 Mass formulae . . . . . . . . . . . . . . . . . . . . . . . . . . . . . . . . . . . . . . . . . . .

7.3 The Israel-Wilson-Perjés class . . . . . . . . . . . . . . . . . . . . . . 44

8 Stationary and Axisymmetric Spacetimes $\quad 46$

8.1 Integrability properties of Killing fields . . . . . . . . . . . . . . . . . . . . . 4 46

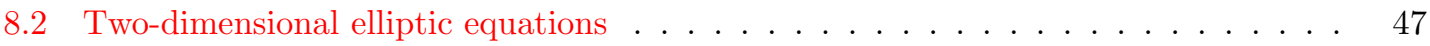

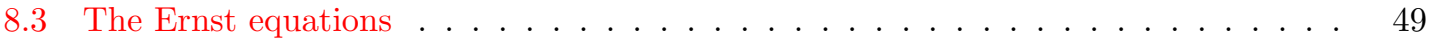

8.3.1 A derivation of the Kerr-Newman metric . . . . . . . . . . . . . . . 49

8.4 The uniqueness theorem for the Kerr-Newman solution . . . . . . . . . . . . . 50

8.4 Divergence identities . . . . . . . . . . . . . . . . . 50

8.4.2 The distance function argument . . . . . . . . . . . . . . 51

9 Acknowledgments $\quad 53$

$\begin{array}{lr}\text { References } & 54\end{array}$ 



\section{Introduction}

\subsection{General remarks}

Our conception of black holes has experienced several dramatic changes during the last two hundred years: While the "dark stars" of Michell [235] and Laplace [210] were merely regarded as peculiarities of Newton's law of gravity and his corpuscular theory of light, black holes are nowadays widely believed to exist in our universe (for a review on the evolution of the subject the reader is referred to Israel's comprehensive account [178]; see also [52, 51]). Although the observations are necessarily indirect, the evidence for both stellar and galactic black holes has become compelling $[275,232,233,247,242,231]$. There seems to be consensus [276, 197, 234, 248] that the two most convincing supermassive black-hole candidates are the galactic nuclei of NGC 4258 and of our own Milky Way [123].

The theory of black holes was initiated by the pioneering work of Chandrasekhar [53, 54] in the early 1930s. (However, the geometry of the Schwarzschild solution [290, 291] was misunderstood for almost half a century; the misconception of the "Schwarzschild singularity" was retained until the late 1950s.) Computing the Chandrasekhar limit for neutron stars [8], Oppenheimer and Snyder [257], and Oppenheimer and Volkoff [258] were able to demonstrate that black holes present the ultimate fate of sufficiently-massive stars. Modern black-hole physics started with the advent of relativistic astrophysics, in particular with the discovery of pulsars in 1967.

One of the most intriguing outcomes of the mathematical theory of black holes is the uniqueness theorem, applying to a class of stationary solutions of the Einstein-Maxwell equations. Strikingly enough, its consequences can be made into a test of general relativity [285]. The assertion, that all (four-dimensional) electrovacuum black-hole spacetimes are characterized by their mass, angular momentum and electric charge, is strangely reminiscent of the fact that a statistical system in thermal equilibrium is described by a small set of state variables as well, whereas considerably more information is required to understand its dynamical behavior. The similarity is reinforced by the black-hole-mass-variation formula [9] and the area-increase theorem [143, 69], which are analogous to the corresponding laws of ordinary thermodynamics. These mathematical relationships are given physical significance by the observation that the temperature of the black body spectrum of the Hawking radiation [142] is equal to the surface gravity of the black hole. There has been steady interest in the relationship between the laws of black hole mechanics and the laws of thermodynamics. In particular, computations within string theory seem to offer a promising interpretation of black-hole entropy [171]. The reader interested in the thermodynamic properties of black holes is referred to the review by Wald [316].

There has been substantial progress towards a proof of the celebrated uniqueness theorem, conjectured by Israel, Penrose and Wheeler in the late sixties [76, 79, 217] during the last four decades (see, e.g., [58] and [59] for previous reviews). Some open gaps, notably the electrovacuum staticity theorem [302, 303] and the topology theorems [109, 110, 85], have been closed (see [59, $73,65]$ for related new results). Early on, the theorem led to the expectation that the stationaryblack-hole solutions of other self-gravitating matter fields might also be parameterized by their mass, angular momentum and a set of charges (generalized no-hair conjecture). However, ever since Bartnik and McKinnon discovered the first self-gravitating Yang-Mills soliton in 1988 [14], a variety of new black hole configurations have been found, which violate the generalized no-hair conjecture, that suitably regular black-hole spacetimes are classified by a finite set of asymptoticallydefined global charges. These solutions include non-Abelian black holes [310, 208, 24], as well as black holes with Skyrme [94, 161], Higgs [28, 254, 140] or dilaton fields [212, 132].

In fact, black-hole solutions with hair were already known before 1989: in 1982, Gibbons found a black-hole solution with a non-trivial dilaton field, within a model occurring in the low energy limit of $N=4$ supergravity [126]. 
While the above counterexamples to the no-hair conjecture consist of static, spherically-symmetric configurations, there exists numerical evidence that static black holes are not necessarily spherically symmetric [192, 93]; in fact, they might not even need to be axisymmetric [278]. Moreover, some studies also indicate that non-rotating black holes need not be static [38]. The rich spectrum of stationary-black-hole configurations demonstrates that the matter fields are by far more critical to the properties of black-hole solutions than expected for a long time. In fact, the proof of the uniqueness theorem is, at least in the axisymmetric case, heavily based on the fact that the Einstein-Maxwell equations in the presence of a Killing symmetry form a $\sigma$-model, effectively coupled to three-dimensional gravity [250]. ( $\sigma$-models are a special case of harmonic maps, and we will use both terminologies interchangeably in our context.) Since this property is not shared by models with non-Abelian gauge fields [35], it is, with hindsight, not too surprising that the Einstein-Yang-Mills system admits black holes with hair.

However, there exist other black hole solutions, which are likely to be subject to a generalized version of the uniqueness theorem. These solutions appear in theories with self-gravitating massless scalar fields (moduli) coupled to Abelian gauge fields. The expectation that uniqueness results apply to a variety of these models arises from the observation that their dimensional reduction (with respect to a Killing symmetry) yields a $\sigma$-model with symmetric target space (see, e.g., [31, 86, 120], and references therein).

\subsection{Organization}

The purpose of this text is to review some features of four-dimensional stationary asymptoticallyflat black-hole spacetimes. Some black-hole solutions with non-zero cosmological constant can be found in $[313,36,323,286,271,15]$. It should be noted that the discovery of five-dimensional black rings by Emparan and Reall [99] has given new life to the overall subject (see [100,101] and references therein) but here we concentrate on four-dimensional spacetimes with mostly classical matter fields.

For detailed introductions into the subject we refer to Chandrasekhar's book on the mathematical theory of black holes [56], the classic textbook by Hawking and Ellis [143], Carter's review [50], Chapter 12 of Wald's book [314], the overview [63] and the monograph [151].

The first part of this report is intended to provide a guide to the literature, and to present some of the main issues, without going into technical details. We start by collecting the significant definitions in Section 2. We continue, in Section 3, by recalling the main steps leading to the uniqueness theorem for electro-vacuum black-hole spacetimes. The classification scheme obtained in this way is then reexamined in the light of solutions, which are not covered by no-hair theorems, such as stationary Kaluza-Klein black holes (Section 4) and the Einstein-Yang-Mills black holes (Section 5).

The second part reviews the main structural properties of stationary black-hole spacetimes. In particular, we discuss the dimensional reduction of the field equations in the presence of a Killing symmetry in more detail (Section 6). For a variety of matter models, such as self-gravitating Abelian gauge fields, the reduction yields a $\sigma$-model, with symmetric target manifold, coupled to three-dimensional gravity. In Section 7 we discuss some aspects of this structure, namely the Mazur identity and the quadratic mass formulae, and we present the Israel-Wilson class of metrics.

The third part is devoted to stationary and axisymmetric black-hole spacetimes (Section 8). We start by recalling the circularity problem for non-Abelian gauge fields and for scalar mappings. The dimensional reduction with respect to the second Killing field leads to a boundary value problem on a fixed, two-dimensional background. As an application, we outline the uniqueness proof for the Kerr-Newman metric. 


\section{Definitions}

It is convenient to start with definitions, which will be grouped together in separate sections.

\subsection{Asymptotic flatness}

We will mostly be concerned with asymptotically-flat black holes. A spacetime $(M, \mathbf{g})$ will be said to possess an asymptotically-flat end if $M$ contains a spacelike hypersurface $\mathscr{S}_{\text {ext }}$ diffeomorphic to $\mathbb{R}^{n} \backslash B(R)$, where $B(R)$ is an open coordinate ball of radius $R$, with the following properties: there exists a constant $\alpha>0$ such that, in local coordinates on $\mathscr{S}_{\text {ext }}$ obtained from $\mathbb{R}^{n} \backslash B(R)$, the metric $\gamma$ induced by $\mathbf{g}$ on $\mathscr{S}_{\text {ext }}$, the extrinsic curvature tensor $K_{i j}$ of $\mathscr{S}_{\text {ext }}$, and the electromagnetic potential $A_{\mu}$ satisfy the fall-off conditions

$$
\gamma_{i j}-\delta_{i j}=O_{d}\left(r^{-\alpha}\right), \quad K_{i j}=O_{d-1}\left(r^{-1-\alpha}\right),
$$

and

$$
A_{\mu}=O_{d}\left(r^{-\alpha}\right),
$$

for some $d>1$, where we write $f=O_{d}\left(r^{\alpha}\right)$ if $f$ satisfies

$$
\partial_{i_{1}} \ldots \partial_{i_{\ell}} f=O\left(r^{\alpha-\ell}\right), \quad 0 \leq \ell \leq d .
$$

\subsection{Kaluza-Klein asymptotic flatness}

There exists a generalization of the notion of asymptotic flatness, which is relevant to both fourand higher-dimensional gravitation. We shall say that $\mathscr{S}_{\text {ext }}$ is a Kaluza-Klein asymptotic end if $\mathscr{S}_{\text {ext }}$ is diffeomorphic to $\left(\mathbb{R}^{N} \backslash \bar{B}(R)\right) \times Q$, where $\bar{B}(R)$ is a closed coordinate ball of radius $R$ and $Q$ is a compact manifold of dimension $s \geq 0$; a spacetime containing such an end is said to have $N+1$ asymptotically-large dimensions. Let $h$ be a fixed Riemaniann metric on $Q$, and let $\stackrel{\circ}{g}=\delta \oplus \stackrel{\circ}{h}$, where $\delta$ is the Euclidean metric on $\mathbb{R}^{N}$. A spacetime $(M, \mathbf{g})$ containing such an end will be said to be Kaluza-Klein asymptotically flat, or KK-asymptotically flat if, for some $\alpha>0$, the metric $\gamma$ induced by $\mathbf{g}$ on $\mathscr{S}_{\text {ext }}$ and the extrinsic curvature tensor $K_{i j}$ of $\mathscr{S}_{\text {ext }}$, satisfy the fall-off conditions

$$
\gamma_{i j}-\stackrel{\circ}{g}_{i j}=O_{d}\left(r^{-\alpha-l}\right), \quad K_{i j}=O_{d-1}\left(r^{-1-\alpha-l}\right),
$$

where, in this context, $r$ is the radius in $\mathbb{R}^{N}$ and we write $f=O_{d}\left(r^{\alpha}\right)$ if $f$ satisfies

$$
\stackrel{\circ}{D}_{i_{1}} \ldots \stackrel{\circ}{D}_{i_{l}} f=O\left(r^{\alpha-l}\right), \quad 0 \leq \ell \leq d,
$$

with $\stackrel{\circ}{D}$ the Levi-Civita connection of $\stackrel{\circ}{g}$.

\subsection{Stationary metrics}

An asymptotically-flat, or $K K$-asymptotically-flat, spacetime $(M, \mathbf{g})$ will be called stationary if there exists on $M$ a complete Killing vector field $k$, which is timelike in the asymptotic region $\mathscr{S}_{\text {ext }}$; such a Killing vector will be sometimes called stationary as well. In fact, in most of the literature it is implicitly assumed that stationary Killing vectors satisfy $\mathbf{g}(k, k)<-\epsilon<0$ for some $\epsilon$ and for all $r$ large enough. This uniformity condition excludes the possibility of a timelike vector, which asymptotes to a null one. This involves no loss of generality in well-behaved asymptotically-flat spacetimes: indeed, this uniform timelikeness condition always holds for Killing vectors, which are timelike for all large distances if the conditions of the positive energy theorem are met [17, 77]. 
In electrovacuum, as part of the definition of stationarity it is also required that the Maxwell field be invariant with respect to $k$, that is

$$
L_{k} F \equiv 0
$$

Note that this definition assumes that the Killing vector $k$ is complete, which means that for every $p \in M$ the orbit $\phi_{t}[k](p)$ of $k$ is defined for all $t \in \mathbb{R}$. The question of completeness of Killing vectors is an important issue, which needs justifying in some steps of the uniqueness arguments [57, 59].

In regions where $k$ is timelike, there exist local coordinates in which the metric takes the form

$$
\mathbf{g}=-V^{2}(d t+\underbrace{\theta_{i} d x^{i}}_{=: \theta})^{2}+\underbrace{\gamma_{i j} d x^{i} d x^{j}}_{=: \gamma},
$$

with

$$
k=\partial_{t} \quad \Longrightarrow \quad \partial_{t} V=\partial_{t} \theta_{i}=\partial_{t} \gamma_{i j}=0 \text {. }
$$

Such coordinates exist globally on asymptotically-flat ends, and if the Einstein-Maxwell equations hold, one can also obtain there [58, Section 1.3], in dimension $3+1$,

$$
\gamma_{i j}-\delta_{i j}=O_{\infty}\left(r^{-1}\right), \quad \theta_{i}=O_{\infty}\left(r^{-1}\right), \quad V-1=O_{\infty}\left(r^{-1}\right),
$$

and

$$
A_{\mu}=O_{\infty}\left(r^{-1}\right),
$$

where the infinity symbol means that (2.3) holds for arbitrary $d$.

\subsection{Domains of outer communications, event horizons}

For $t \in \mathbb{R}$ let $\phi_{t}[k]: M \rightarrow M$ denote the one-parameter group of diffeomorphisms generated by $k$; we will write $\phi_{t}$ for $\phi_{t}[k]$ whenever ambiguities are unlikely to occur.

Recall that $I^{-}(\Omega)$, respectively $J^{-}(\Omega)$, is the set covered by past-directed timelike, respectively causal, curves originating from $\Omega$, while $\dot{I}^{-}$denotes the boundary of $I^{-}$, etc. The sets $I^{+}$, etc., are defined as $I^{-}$, etc., after changing time-orientation. See [143, 16, 256, 236, 266, 66] and references therein for details of causality theory.

Consider an asymptotically-flat, or $K K$-asymptotically-flat, spacetime with a Killing vector $k$, which is timelike on the asymptotic end $\mathscr{S}_{\text {ext }}$. The exterior region $M_{\text {ext }}$ and the domain of outer communications $\left\langle\left\langle M_{\text {ext }}\right\rangle\right\rangle$, for which we will also use the abbreviation d.o.c., are then defined as (see Figure 1)
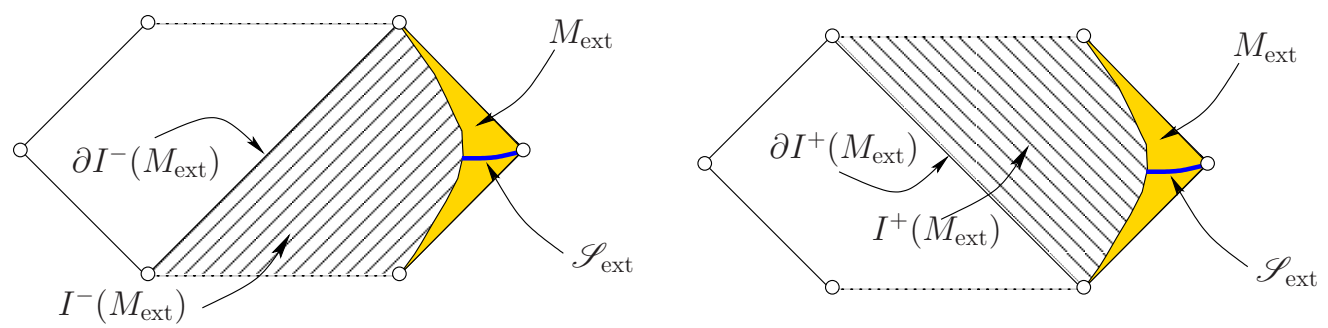

Figure 1: $\mathscr{S}_{\text {ext }}, M_{\text {ext }}$, together with the future and the past of $M_{\text {ext }}$. One has $M_{\text {ext }} \subset I^{ \pm}\left(M_{\text {ext }}\right)$, even though this is not immediately apparent from the figure. The domain of outer communications is the intersection $I^{+}\left(M_{\text {ext }}\right) \cap I^{-}\left(M_{\text {ext }}\right)$; compare with Figure 2 . 


$$
\left\langle\left\langle M_{\text {ext }}\right\rangle\right\rangle=I^{+}(\underbrace{\cup_{t} \phi_{t}\left(\mathscr{S}_{\text {ext }}\right)}_{=: M_{\text {ext }}}) \cap I^{-}\left(\cup_{t} \phi_{t}\left(\mathscr{S}_{\text {ext }}\right)\right) .
$$

The black-hole region $\mathscr{B}$ and the black-hole event horizon $\mathscr{H}^{+}$are defined as

$$
\mathscr{B}=M \backslash I^{-}\left(M_{\text {ext }}\right), \quad \mathscr{H}^{+}=\partial \mathscr{B} .
$$

The white-hole region $\mathscr{W}$ and the white-hole event horizon $\mathscr{H}^{-}$are defined as above after changing time orientation:

$$
\mathscr{W}=M \backslash I^{+}\left(M_{\text {ext }}\right), \quad \mathscr{H}^{-}=\partial \mathscr{W}, \quad \mathscr{H}=\mathscr{H}^{+} \cup \mathscr{H}^{-} .
$$

It follows that the boundaries of $\left\langle\left\langle M_{\text {ext }}\right\rangle\right\rangle$ are included in the event horizons. We set

$$
\mathscr{E}^{ \pm}=\partial\left\langle\left\langle M_{\text {ext }}\right\rangle\right\rangle \cap I^{ \pm}\left(M_{\text {ext }}\right), \quad \mathscr{E}^{\prime}=\mathscr{E}^{+} \cup \mathscr{E}^{-}
$$

There is considerable freedom in choosing the asymptotic region $\mathscr{S}_{\text {ext }}$. However, it is not too difficult to show that $I^{ \pm}\left(M_{\text {ext }}\right)$, and hence $\left\langle\left\langle M_{\text {ext }}\right\rangle\right\rangle, \mathscr{H}^{ \pm}$and $\mathscr{E}^{ \pm}$, are independent of the choice of $\mathscr{S}_{\text {ext }}$ whenever the associated $M_{\text {ext }}$ 's overlap.

By standard causality theory, an event horizon is the union of Lipschitz null hypersurfaces. It turns out that event horizons in stationary spacetimes satisfying energy conditions are as smooth as the metric allows [76, 69]; thus, smooth if the metric is smooth, analytic if the metric is.

\subsection{Killing horizons}

A null embedded hypersurface, invariant under the flow of a Killing vector $k$, which coincides with a connected component of the set

$$
\mathscr{N}[k]:=\{\mathbf{g}(k, k)=0, k \neq 0\},
$$

is called a Killing horizon associated to $k$. We will often write $H[k]$ for $\mathscr{N}[k]$, whenever $\mathscr{N}[k]$ is a Killing horizon.

\subsubsection{Bifurcate Killing horizons}

The Schwarzschild black hole has an event horizon with a specific structure, which is captured by the following definition: A set is called a bifurcate Killing horizon if it is the union of a smooth spacelike submanifold $S$ of co-dimension two, called the bifurcation surface, on which a Killing vector field $k$ vanishes, and of four smooth null embedded hypersurfaces obtained by following null geodesics in the four distinct null directions normal to $S$.

For example, the Killing vector $x \partial_{t}+t \partial_{x}$ in Minkowski spacetime has a bifurcate Killing horizon, with the bifurcation surface $\{t=x=0\}$. As already mentioned, another example is given by the set $\{r=2 m\}$ in Schwarzschild-Kruskal-Szekeres spacetime with positive mass parameter $m$.

In the spirit of the previous definition, we will refer to the union of two null hypersurfaces, which intersect transversally on a 2-dimensional spacelike surface as a bifurcate null surface.

The reader is warned that a bifurcate Killing horizon is not a Killing horizon, as defined in Section 2.5, since the Killing vector vanishes on $S$. If one thinks of $S$ as not being part of the bifurcate Killing horizon, then the resulting set is again not a Killing horizon, since it has more than one component. 


\subsubsection{Killing prehorizons}

One of the key steps of the uniqueness theory, as described in Section 3, forces one to consider "horizon candidates" with local properties similar to those of a proper event horizon, but with global behavior possibly worse: A connected, not necessarily embedded, null hypersurface $H_{0} \subset$ $\mathscr{N}[k]$ to which $k$ is tangent is called a Killing prehorizon. In this terminology, a Killing horizon is a Killing prehorizon, which forms a embedded hypersurface, which coincides with a connected component of $\mathscr{N}[k]$. The Minkowskian Killing vector $\partial_{t}-\partial_{x}$ provides an example where $\mathscr{N}$ is not a hypersurface, with every hyperplane $t+x=$ const being a prehorizon.

The Killing vector $k=\partial_{t}+Y$ on $\mathbb{R} \times \mathbb{T}^{n}$, equipped with the flat metric, where $\mathbb{T}^{n}$ is an $n$ dimensional torus, and where $Y$ is a unit Killing vector on $\mathbb{T}^{n}$ with dense orbits, admits prehorizons, which are not embedded. This last example is globally hyperbolic, which shows that causality conditions are not sufficient to eliminate this kind of behavior.

Of crucial importance to the zeroth law of black-hole physics (to be discussed shortly) is the fact that the $(k, k)$-component of the Ricci tensor vanishes on horizons or prehorizons,

$$
R(k, k)=0 \quad \text { on } \quad H[k] .
$$

This is a simple consequence of the Raychaudhuri equation.

The following two properties of Killing horizons and prehorizons play a role in the theory of stationary black holes:

- A theorem due to Vishveshwara [308] gives a characterization of the Killing horizon $H[k]$ in terms of the twist $\omega$ of $k:{ }^{1}$ A connected component of the set $\mathscr{N}:=\{g(k, k)=0, k \neq 0\}$ is a (non-degenerate) Killing horizon whenever

$$
\omega=0 \quad \text { and } \quad i_{k} \mathrm{~d} k \neq 0 \text { on } \mathscr{N} \text {. }
$$

- The following characterization of Killing prehorizons is often referred to as the VishveshwaraCarter Lemma [46, 43] (compare [61, Addendum]): Let $(M, \mathbf{g})$ be a smooth spacetime with complete, static Killing vector $k$. Then the set $\left\{p \in M|g(k, k)|_{p}=0, k(p) \neq 0\right\}$ is the union of integral leaves of the distribution $k^{\perp}$, which are totally geodesic within $M \backslash\{k=0\}$.

\subsubsection{Surface gravity: degenerate, non-degenerate and mean-non-degenerate hori- zons}

An immediate consequence of the definition of a Killing horizon or prehorizon is the proportionality of $k$ and $\mathrm{d} N$ on $H[k]$, where

$$
N:=\mathbf{g}(k, k) \text {. }
$$

This follows, e.g., from $g(k, \mathrm{~d} N)=0$, since $L_{k} N=0$, and from the fact that two orthogonal null vectors are proportional. The observation motivates the definition of the surface gravity $\kappa$ of a Killing horizon or prehorizon $H[k]$, through the formula

$$
\left.d(\mathbf{g}(k, k))\right|_{H}=-2 \kappa k,
$$

where we use the same symbol $k$ for the covector $\mathbf{g}_{\mu \nu} k^{\nu} d x^{\mu}$ appearing in the right-hand side as for the vector $k^{\mu} \partial_{\mu}$.

The Killing equation implies $\mathrm{d} N=-2 \nabla_{k} k$; we see that the surface gravity measures the extent to which the parametrization of the geodesic congruence generated by $k$ is not affine.

${ }^{1}$ See, e.g., [89], p. 239 or [151], p. 92 for the proof. 
A fundamental property is that the surface gravity $\kappa$ is constant over horizons or prehorizons in several situations of interest. This leads to the intriguing fact that the surface gravity plays a similar role in the theory of stationary black holes as the temperature does in ordinary thermodynamics. Since the latter is constant for a body in thermal equilibrium, the result

$$
\kappa=\text { constant } \quad \text { on } H[k]
$$

is usually called the zeroth law of black-hole physics [9].

The constancy of $\kappa$ holds in vacuum, or for matter fields satisfying the dominant-energy condition, see, e.g., [151, Theorem 7.1]. The original proof of the zeroth law [9] proceeds as follows: First, Einstein's equations and the fact that $R(k, k)$ vanishes on the horizon imply that $T(k, k)=0$ on $H[k]$. Hence, the vector field $T(k):=T^{\mu}{ }_{\nu} k^{\nu} \partial_{x^{\mu}}$ is perpendicular to $k$ and, therefore, space-like (possibly zero) or null on $H[k]$. On the other hand, the dominant energy condition requires that $T(k)$ is zero, time-like or null. Thus, $T(k)$ vanishes or is null on the horizon. Since two orthogonal null vectors are proportional, one has, using Einstein's equations again, $k \wedge R(k)=0$ on $H[k]$, where $R(k)=R_{\mu \nu} k^{\mu} d x^{\nu}$. The result that $\kappa$ is constant over each horizon follows now from the general property (see, e.g., [314])

$$
k \wedge \mathrm{d} \kappa=-k \wedge R(k) \quad \text { on } \quad H[k] .
$$

The proof of (2.16) given in [314] generalizes to all spacetime dimensions $n+1 \geq 4$; the result also follows in all dimensions from the analysis in [165] when the horizon has compact spacelike sections.

By virtue of Eq. (2.17) and the identity $\mathrm{d} \omega=*[k \wedge R(k)]$, the zeroth law follows if one can show that the twist one-form is closed on the horizon [270]:

$$
[\mathrm{d} \omega]_{H[k]}=0 \Longrightarrow \kappa=\text { constant on } H[k] .
$$

While the original proof of the zeroth law takes advantage of Einstein's equations and the dominant energy condition to conclude that the twist is closed, one may also achieve this by requiring that $\omega$ vanishes identically, which then proves the zeroth law under the second set of hypotheses listed below. This is obvious for static configurations, since then $k$ has vanishing twist by definition.

Yet another situation of interest is a spacetime with two commuting Killing vector fields $k$ and $m$, with a Killing horizon $H[\xi]$ associated to a Killing vector $\xi=k+\Omega m$. Such a spacetime is said to be circular if the distribution of planes spanned by $k$ and $m$ is hypersurface-orthogonal. Equivalently, the metric can be locally written in a $2+2$ block-diagonal form, with one of the blocks defined by the orbits of $k$ and $m$. In the circular case one shows that $g\left(m, \omega_{\xi}\right)=g\left(\xi, \omega_{m}\right)=0$ implies $\mathrm{d} \omega_{\xi}=0$ on the horizon generated by $\xi$; see [151], Chapter 7 for details.

A significant observation is that of Kay and Wald [184], that $\kappa$ must be constant on bifurcate Killing horizons, regardless of the matter content. This is proven by showing that the derivative of the surface gravity in directions tangent to the bifurcation surface vanishes. Hence, $\kappa$ cannot vary between the null-generators. But it is clear that $\kappa$ is constant along the generators.

Summarizing, each of the following hypotheses is sufficient to prove that $\kappa$ is constant over a Killing horizon defined by $k$ :

(i) The dominant energy condition holds;

(ii) the domain of outer communications is static;

(iii) the domain of outer communications is circular;

(iv) $H[k]$ is a bifurcate Killing horizon. 
See [270] for some further observations concerning (2.16).

A Killing horizon is called degenerate if $\kappa$ vanishes, and non-degenerate otherwise.

As an example, in Minkowski spacetime, consider the Killing vector $\xi=x \partial_{t}+t \partial_{x}$. We have

$$
d(\mathbf{g}(\xi, \xi))=d\left(-x^{2}+t^{2}\right)=2(-x d x+t d t),
$$

which equals twice $\xi^{b}:=\mathbf{g}_{\mu \nu} \xi^{\mu} d x^{\nu}$ on each of the four Killing horizons

$$
H(\xi)_{\epsilon \delta}:=\{t=\epsilon x, \delta t>0\}, \quad \epsilon, \delta \in\{ \pm 1\} .
$$

On the other hand, for the Killing vector

$$
k=y \partial_{t}+t \partial_{y}+x \partial_{y}-y \partial_{x}=y \partial_{t}+(t+x) \partial_{y}-y \partial_{x}
$$

one obtains

$$
d(\mathbf{g}(k, k))=2(t+x)(d t+d x),
$$

which vanishes on each of the Killing horizons $\{t=-x, y \neq 0\}$. This shows that the same null surface can have zero or non-zero values of surface gravity, depending upon which Killing vector has been chosen to calculate $\kappa$.

A key theorem of Rácz and Wald [270] asserts that non-degenerate horizons (with a compact cross section and constant surface gravity) are "essentially bifurcate", in the following sense: Given a spacetime with such a non-degenerate Killing horizon, one can find another spacetime, which is locally isometric to the original one in a one-sided neighborhood of a subset of the horizon, and which contains a bifurcate Killing horizon. The result can be made global under suitable conditions.

The notion of average surface gravity can be defined for null hypersurfaces, which are not necessarily Killing horizons: Following [238], near a smooth null hypersurface $\mathscr{N}$ one can introduce Gaussian null coordinates, in which the metric takes the form

$$
\mathbf{g}=r \varphi d v^{2}+2 d v d r+2 r h_{a} d x^{a} d v+h_{a b} d x^{a} d x^{b} .
$$

The null hypersurface $\mathscr{N}$ is given by the equation $\{r=0\}$; when it corresponds to an event horizon, by replacing $r$ by $-r$ if necessary we can, without loss of generality, assume that $r>0$ in the domain of outer communications. Assuming that $\mathscr{N}$ admits a smooth compact cross-section $S$, the average surface gravity $\langle\kappa\rangle_{S}$ is defined as

$$
\langle\kappa\rangle_{S}=-\frac{1}{|S|} \int_{S} \varphi d \mu_{h},
$$

where $d \mu_{h}$ is the measure induced by the metric $h$ on $S$, and $|S|$ is the area of $S$. We emphasize that this is defined regardless of whether or not the hypersurface is a Killing horizon; but if it is with respect to a vector $k$, and if the surface gravity $\kappa$ of $k$ is constant on $S$, then $\langle\kappa\rangle_{S}$ equals $\kappa$.

A smooth null hypersurface, not necessarily a Killing horizon, with a smooth compact crosssection $S$ such that $\langle\kappa\rangle_{S} \neq 0$ is said to be mean non-degenerate.

Using general identities for Killing fields (see, e.g., [151], Chapter 2) one can derive the following explicit expressions for $\kappa$ :

$$
\kappa^{2}=-\lim _{N \rightarrow 0}\left[\frac{1}{N} g\left(\nabla_{k} k, \nabla_{k} k\right)\right]=-\left[\frac{1}{4} \Delta_{\mathbf{g}} N\right]_{H[k]},
$$

where $\Delta_{\mathbf{g}}$ denotes the Laplace-Beltrami operator of the metric $\mathbf{g}$. Introducing the four velocity $u=k / \sqrt{-N}$ for a time-like $k$, the first expression shows that the surface gravity is the limiting value of the force applied at infinity to keep a unit mass at $H[k]$ in place: $\kappa=\lim (\sqrt{-N}|a|)$, where $a=\nabla_{u} u$ (see, e.g., [314]). 


\section{$2.6 \quad I^{+}$-regularity}

The classification theory of stationary black holes requires that the spacetime under consideration satisfies various global regularity conditions. These are captured by the following definition:

Definition 2.1 Let $(M, \mathbf{g})$ be a spacetime containing an asymptotically-flat end, or a KK-asymptotically-flat end $\mathscr{S}_{\text {ext }}$, and let $k$ be a stationary Killing vector field on $M$. We will say that $(M, \mathbf{g}, k)$ is $I^{+}$-regular if $k$ is complete, if the domain of outer communications $\left\langle\left\langle M_{\mathrm{ext}}\right\rangle\right\rangle$ is globally hyperbolic, and if $\left\langle\left\langle M_{\mathrm{ext}}\right\rangle\right\rangle$ contains a spacelike, connected, acausal hypersurface $\mathscr{S} \supset \mathscr{S}_{\text {ext }}$, the closure $\overline{\mathscr{S}}$ of which is a topological manifold with boundary, consisting of the union of a compact set and of a finite number of asymptotic ends, such that the boundary $\partial \overline{\mathscr{S}}:=\overline{\mathscr{S}} \backslash \mathscr{S}$ is a topological manifold satisfying

$$
\partial \overline{\mathscr{S}} \subset \mathscr{E}^{+}:=\partial\left\langle\left\langle M_{\mathrm{ext}}\right\rangle\right\rangle \cap I^{+}\left(M_{\mathrm{ext}}\right),
$$

with $\partial \overline{\mathscr{S}}$ meeting every generator of $\mathscr{E}^{+}$precisely once. (See Figure 2.)

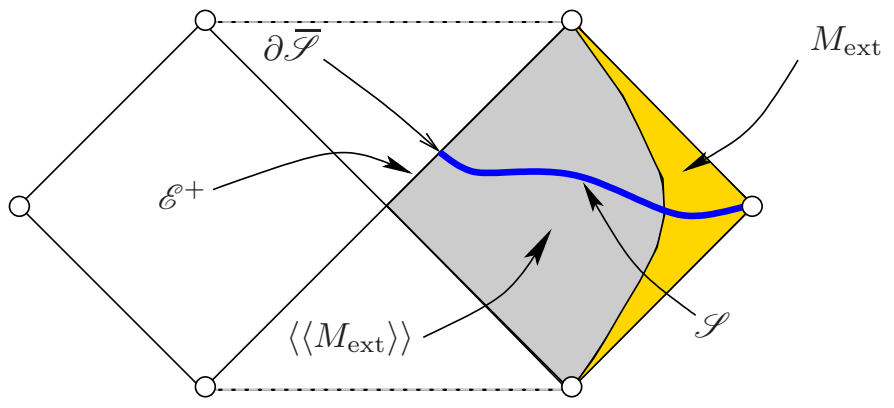

Figure 2: The hypersurface $\mathscr{S}$ from the definition of $I^{+}$-regularity.

The " $I^{+}$" of the name is due to the $I^{+}$appearing in (2.23).

Some comments about the definition are in order. First, one requires completeness of the orbits of the stationary Killing vector to have an action of $\mathbb{R}$ on $M$ by isometries. Next, global hyperbolicity of the domain of outer communications is used to guarantee its simple connectedness, to make sure that the area theorem holds, and to avoid causality violations as well as certain kinds of naked singularities in $\left\langle\left\langle M_{\text {ext }}\right\rangle\right\rangle$. Further, the existence of a well-behaved spacelike hypersurface is a prerequisite to any elliptic PDEs analysis, as is extensively needed for the problem at hand. The existence of compact cross-sections of the future event horizon prevents singularities on the future part of the boundary of the domain of outer communications, and eventually guarantees the smoothness of that boundary. The requirement Eq. (2.23) might appear somewhat unnatural, as there are perfectly well-behaved hypersurfaces in, e.g., the Schwarzschild spacetime, which do not satisfy this condition, but there arise various technical difficulties without this condition. Needless to say, all those conditions are satisfied by the Kerr-Newman and the Majumdar-Papapetrou (MP) solutions. 


\section{Towards a classification of stationary electrovacuum black hole spacetimes}

While the uniqueness theory for black-hole solutions of Einstein's vacuum equations and the Einstein-Maxwell (EM) equations has seen deep successes, the complete picture is nowhere settled at the time of revising of this work. We know now that, under reasonable global conditions (see Definition 2.1), the domains of dependence of analytic, stationary, asymptotically-flat electrovacuum black-hole spacetimes with a connected non-degenerate horizon belong to the Kerr-Newman family. The purpose of this section is to review the various steps involved in the classification of electrovacuum spacetimes (see Figure 3). In Section 5, we shall then comment on the validity of the partial results in the presence of non-linear matter fields.

For definiteness, from now on we assume that all spacetimes are $I^{+}$-regular. We note that the slightly weaker global conditions spelled-out in Theorem 3.1 suffice for the analysis of static spacetimes, or for various intermediate steps of the uniqueness theory, but those weaker conditions are not known to suffice for the Uniqueness Theorem 3.3.

The main task of the uniqueness program is to show that the domains of outer communications of sufficiently regular stationary electrovacuum black-hole spacetimes are exhausted by the KerrNewman or the MP spacetimes.

The starting point is the smoothness of the event horizon; this is proven in [76, Theorem 4.11], drawing heavily on the results in [69].

One proves, next, that connected components of the event horizon are diffeomorphic to $\mathbb{R} \times S^{2}$. This was established in [85], taking advantage of the topological censorship theorem of Friedman, Schleich and Witt [106]; compare [141] for a previous partial result. (Related versions of the topology theorem, applying to globally-hyperbolic, not-necessarily-stationary, spacetimes, have been established by Jacobson and Venkataramani [180], and by Galloway [108, 109, 110, 112]; the strongest-to-date version, with very general asymptotic hypotheses, can be found in [73].)

\subsection{Static solutions}

A stationary spacetime is called static if the Killing vector $k$ is hypersurface-orthogonal: this means that the distribution of the hyperplanes orthogonal to $k$ is integrable. Equivalently,

$$
k \wedge d k=0 .
$$

Here and elsewhere, by a common abuse of notation, we also write $k$ for the one-form associated with $k$.

The results concerning static black holes are stronger than the general stationary case, and so this case deserves separate discussion. In any case, the proof of uniqueness for stationary black holes branches out at some point and one needs to consider separately uniqueness for static configurations.

In pioneering work, Israel showed that both static vacuum [176] and electrovacuum [177] blackhole spacetimes satisfying a set of restrictive conditions are spherically symmetric. Israel's ingenious method, based on differential identities and Stokes' theorem, triggered a series of investigations devoted to the static uniqueness problem (see, e.g., [244, 245, 279, 281, 294]). A breakthrough was made by Bunting and Masood-ul-Alam [42], who showed how to use the positive energy theorem ${ }^{2}$

2 This theorem was first proven by Schoen and Yau [288, 289] and somewhat later, using spinor techniques, by Witten [325] (compare [265]). See [12] for a version relevant to the uniqueness problem, which allows degenerate components of the event horizon. 
to exclude non-connected configurations (compare [61]). ${ }^{3}$

The annoying hypothesis of analyticity, which was implicitly assumed in the above treatments, has been removed in [72]. The issue here is to show that the Killing vector field cannot become null on the domain of outer communications. The first step to prove this is the Vishveshwara-Carter lemma (see Section 2.5.2 and [308, 43]), which shows that null orbits of static Killing vectors form a prehorizon, as defined in Section 2.5.2. To finish the proof one needs to show that prehorizons cannot occur within the d.o.c. This presents no difficulty when analyticity is assumed. Now, analyticity of stationary electrovacuum metrics is a standard property [245, 243] when the Killing vector is timelike, but timelikeness throughout the d.o.c. is not known yet at this stage of the argument. The nonexistence of prehorizons within the d.o.c. for smooth metrics requires more work, and is the main result in [72].

In the static vacuum case the remainder of the argument can be simplified by noting that there are no static solutions with degenerate horizons, which have spherical cross-sections [81]. This is not true anymore in the electrovacuum case, where an intricate argument to handle non-degenerate horizons is needed [83] (compare [284, 295, 225, 62] for previous partial results).

All this can be summarized in the following classification theorem:

TheOREM 3.1 Let $(M, \mathbf{g})$ be an electrovacuum, four-dimensional spacetime containing a spacelike, connected, acausal hypersurface $\mathscr{S}$, such that $\overline{\mathscr{S}}$ is a topological manifold with boundary consisting of the union of a compact set and of a finite number of asymptotically-flat ends. Suppose that there exists on $M$ a complete hypersurface-orthogonal Killing vector, that the domain of outer communication $\left\langle\left\langle M_{\mathrm{ext}}\right\rangle\right\rangle$ is globally hyperbolic, and that $\partial \overline{\mathscr{S}} \subset M \backslash\left\langle\left\langle M_{\mathrm{ext}}\right\rangle\right\rangle$. Then $\left\langle\left\langle M_{\mathrm{ext}}\right\rangle\right\rangle$ is isometric to the domain of outer communications of a Reissner-Nordström or a MP spacetime.

\subsection{Stationary-axisymmetric solutions}

\subsubsection{Topology}

A second class of spacetimes where reasonably satisfactory statements can be made is provided by stationary-axisymmetric solutions. Here one assumes from the outset that, in addition to the stationary Killing vector, there exists a second Killing vector field. Assuming $I^{+}$-regularity, one can invoke the positive energy theorem to show $[18,19]$ that some linear combination of the Killing vectors, say $m$, must have periodic orbits, and an axis of rotation, i.e., a two-dimensional totally-geodesic submanifold of $M$ on which the Killing vector $m$ vanishes. The description of the quotient manifold is provided by the deep mathematical results concerning actions of isometry groups of $[259,273]$, together with the simple-connectedness and structure theorems [76]. The bottom line is that the spacetime is the product of $\mathbb{R}$ with $\mathbb{R}^{3}$ from which a finite number of aligned balls, each corresponding to a black hole, has been removed. Moreover, the $U(1)$ component of the group of isometries acts by rotations of $\mathbb{R}^{3}$. Equivalently, the quotient space is a half-plane from which one has removed a finite number of disjoint half-discs centered on points lying on the boundary of the half-plane.

\subsubsection{Candidate metrics}

The only known $I^{+}$-regular stationary and axisymmetric solutions of the Einstein-Maxwell equations are the Kerr-Newman metrics and the MP metrics. However, it should be kept in mind that candidate solutions for non-connected black-hole configurations exist:

\footnotetext{
3 Non-existence of certain static $n$-body configurations (possibly, but not necessarily, black holes) was established in [21, 20]). These results rely on the positive energy theorem and exclude, in particular, suitably regular configurations with a reflection symmetry across a noncompact surface, which is disjoint from the matter regions.
} 
First, there are the multi-soliton metrics constructed using inverse scattering methods [23, 22] (compare [268]). Closely related (and possibly identical, see [148]), are the multi-Kerr solutions constructed by successive Bäcklund transformations starting from Minkowski spacetime; a special case is provided by the Neugebauer-Kramer double-Kerr solutions [198]. These are explicit solutions, with the metric functions being rational functions of coordinates and of many parameters. It is known that some subsets of those parameters lead to metrics, which are smooth at the axis of rotation, but one suspects that those metrics will be nakedly singular away from the axis. We will return to that question in Section 3.4.3.

Next, there are the solutions constructed by Weinstein [322], obtained from an abstract existence theorem for suitable harmonic maps. The resulting metrics are smooth everywhere except perhaps at some components of the axis of rotation. It is known that some Weinstein solutions have conical singularities $[319,216,249,70]$ on the axis, but the general case remains open.

Finally, the Israel-Wilson-Perjés (IWP) metrics [267, 179], discussed in more detail in Section 7.3, provide candidates for rotating generalizations of the MP black holes. Those metrics are remarkable because they admit nontrivial Killing spinors. The Killing vector obtained from the Killing spinor is causal everywhere, so the horizons are necessarily non-rotating and degenerate. It has been shown in [80] that the only regular IWP metrics with a Killing vector timelike throughout the d.o.c. are the MP metrics. A strategy for a proof of timelikeness has been given in [80], but the details have yet to be provided. In any case, one expects that the only regular IWP metrics are the MP ones.

Some more information concerning candidate solutions with non-connected horizons can be found in Section 3.4.3.

\subsubsection{The reduction}

Returning to the classification question, the analysis continues with the circularity theorem of Papapetrou [264] and Kundt and Trümper [201] (compare [43]), which asserts that, locally and away from null orbits, the metric of a vacuum or electrovacuum spacetime can be written in a $2+2$ block-diagonal form.

The next key observation of Carter is that the stationary and axisymmetric EM equations can be reduced to a two-dimensional boundary value problem [45] (see Sections 6.1 and 8.2 for more details), provided that the area density of the orbits of the isometry group can be used as a global spacelike coordinate on the quotient manifold. (See also [47] and [50].) Prehorizons intersecting the d.o.c. provide one of the obstructions to this, and a heavy-duty proof that such prehorizons do not arise was given in [76]; a simpler argument has been provided in [72].

In essence, Carter's reduction proceeds through a global manifestly-conformally-flat ("isothermal") coordinate system $(\rho, z)$ on the quotient manifold. One also needs to carefully monitor the boundary conditions satisfied by the fields of interest. The proof of existence of the $(\rho, z)$ coordinates, with sufficient control of the boundary conditions so that the uniqueness proof goes through, has been given in [76], drawing heavily on [64], assuming that all horizons are non-degenerate. A streamlined argument has been presented in [79], where the analysis has also been extended to cover configurations with degenerate components.

So, at this stage one has reduced the problem to the study of solutions of harmonic-type equations on $\mathbb{R}^{3} \backslash \mathscr{A}$, where $\mathscr{A}$ is the rotation axis $\{x=y=0\}$, with precise boundary conditions at the axis. Moreover, the solution is supposed to be invariant under rotations. Equivalently, one has to study a set of harmonic-type equations on a half-plane with specific singularity structure on the boundary.

There exist today at least three arguments that finish the proof, to be described in the following subsections. 


\subsubsection{The Robinson-Mazur proof}

In the vacuum case, Robinson was able to construct an amazing identity, by virtue of which the uniqueness of the Kerr metric followed [280]. The uniqueness problem with electro-magnetic fields remained open until Mazur [228] obtained a generalization of the Robinson identity in a systematic way: The Mazur identity (see also [229, 230, 48, 31, 168, 167]) is based on the observation that the EM equations in the presence of a Killing field describe a non-linear $\sigma$-model with coset space $G / H=S U(1,2) / S(U(1) \times U(2))$. The key to the success is Carter's idea to carry out the dimensional reduction of the EM action with respect to the axial Killing field. Within this approach, the Robinson identity loses its enigmatic status - it turns out to be the explicit form of the Mazur identity for the vacuum case, $G / H=S U(1,1) / U(1)$.

Reduction of the EM action with respect to the time-like Killing field yields, instead, $H=$ $S(U(1,1) \times U(1))$, but the resulting equations become singular on the ergosurface, where the Killing vector becomes null.

More information on this subject is provided in Sections 7.1 and 8.4.1.

\subsubsection{The Bunting-Weinstein harmonic-map argument}

At about the same time, and independently of Mazur, Bunting [41] gave a proof of uniqueness of the relevant harmonic-map equations exploiting the fact that the target space for the problem at hand is negatively curved. A further systematic PDE study of the associated harmonic maps has been carried out by Weinstein: as already mentioned, Weinstein provided existence results for multi-horizon configurations, as well as uniqueness results [322].

All the uniqueness results presented above require precise asymptotic control of the harmonic map and its derivatives at the singular set $\mathscr{A}$. This is an annoying technicality, as no detailed study of the behavior of the derivatives has been presented in the literature. The approach in $[75$, Appendix C] avoids this problem, by showing that a pointwise control of the harmonic map is enough to reach the desired conclusion.

For more information on this subject consult Section 8.4.2.

\subsubsection{The Varzugin-Neugebauer-Meinel argument}

The third strategy to conclude the uniqueness proof has been advocated by Varzugin [306, 307] and, independently, by Neugebauer and Meinel [251]. The idea is to exploit the properties of the linear problem associated with the harmonic map equations, discovered by Belinski and Zakharov [23, 22] (see also [268]). This proceeds by showing that a regular black-hole solution must necessarily be one of the multi-soliton solutions constructed by the inverse-scattering methods, providing an argument for uniqueness of the Kerr solution within the class. Thus, one obtains an explicit form of the candidate metric for solutions with more components, as well as an argument for the non-existence of two-component configurations [249] (compare [70]).

\subsubsection{The axisymmetric uniqueness theorem}

What has been said so far can be summarized as follows:

Theorem 3.2 Let $(M, \mathbf{g})$ be a stationary, axisymmetric asymptotically-flat, $I^{+}$-regular, electrovacuum four-dimensional spacetime. Then the domain of outer communications $\left\langle\left\langle M_{\mathrm{ext}}\right\rangle\right\rangle$ is isometric to one of the Weinstein solutions. In particular, if the event horizon is connected, then $\left\langle\left\langle M_{\mathrm{ext}}\right\rangle\right\rangle$ is isometric to the domain of outer communications of a Kerr-Newman spacetime. 


\subsection{The no-hair theorem}

\subsubsection{The rigidity theorem}

Throughout this section we will assume that the spacetime is $I^{+}$-regular, as made precise by Definition 2.1.

To prove uniqueness of connected, analytic, non-degenerate configurations, it remains to show that every such black hole is either static or axially symmetric. The first step for this is provided by Hawking's strong rigidity theorem (SRT) [143, 238, 60, 107], which relates the global concept of the event horizon to the independently-defined, and logically-distinct, local notion of the Killing horizon. Assuming analyticity, SRT asserts that the event horizon of a stationary black-hole spacetime is a Killing horizon. (In this terminology [151], the weak rigidity theorem is the existence, already discussed above, of prehorizons for static or stationary and axisymmetric configurations.)

A Killing horizon is called non-rotating if it is generated by the stationary Killing field, and rotating otherwise. At this stage the argument branches-off, according to whether at least one of the horizons is rotating, or not.

In the rotating case, Hawking's theorem actually provides only a second Killing vector field defined near the Killing horizon, and to continue one needs to globalize the Killing vector field, to prove that its orbits are complete, and to show that there exists a linear combination of Killing vector fields with periodic orbits and an axis of rotation. This is done in [60], assuming analyticity, drawing heavily on the results in $[253,57,18]$.

The existing attempts in the literature to construct a second Killing vector field without assuming analyticity have only had limited success. One knows now how to construct a second Killing vector in a neighborhood of non-degenerate horizons for electrovacuum black holes [2, 174, 327], but the construction of a second Killing vector throughout the d.o.c. has only been carried out for vacuum near-Kerr non-degenerate configurations so far [3] (compare [326]).

In any case, sufficiently regular analytic stationary electro-vacuum spacetimes containing a rotating component of the event horizon are axially symmetric as well, regardless of degeneracy and connectedness assumptions (for more on this subject see Section 3.4.2). One can then finish the uniqueness proof using Theorem 3.2. Note that the last theorem requires neither analyticity nor connectedness, but leaves open the question of the existence of naked singularities in non-connected candidate solutions.

In the non-rotating case, one continues by showing [84] that the domain of outer communications contains a maximal Cauchy surface. This has been proven so far only for non-degenerate horizons, and this is the only missing step to include situations with degenerate components of the horizon. This allows one to prove the staticity theorem [302, 303], that the stationary Killing field of a non-rotating, electrovacuum black-hole spacetime is hypersurface orthogonal. (Compare [134, 136, $143,141]$ for previous partial results.) One can then finish the argument using Theorem 3.1.

\subsubsection{The uniqueness theorem}

All this leads to the following precise statement, as proven in [76, 79] in vacuum and in [217, 79] in electrovacuum:

THEOREM 3.3 Let $(M, \mathbf{g})$ be a stationary, asymptotically-flat, $I^{+}$-regular, electrovacuum, fourdimensional analytic spacetime. If the event horizon is connected and either mean non-degenerate or rotating, then $\left\langle\left\langle M_{\text {ext }}\right\rangle\right\rangle$ is isometric to the domain of outer communications of a Kerr-Newman spacetime.

The structure of the proof can be summarized in the flow chart of Figure 3. This is to be compared with Figure 4, which presents in more detail the weaker hypotheses needed for various parts of the argument. 
Stationary, analytic, connected, mean non-degenerate, $I^{+}$-regular electrovacuum black-hole spacetime

[asymptotically time-like Killing field $k^{\mu}$ ]

Smoothness and topology theorems

\section{STRONG RIGIDITY THM (1 ${ }^{\text {st }}$ part $)$}

Event horizon = Killing horizon $H[\xi]$

[null generator Killing field $\xi^{\mu}$ ]

$H[\xi]$ non-rotating: $\left.k^{\mu} k_{\mu}\right|_{H[\xi]} \equiv 0$

$H[\xi]$ rotating: $\left.k^{\mu} k_{\mu}\right|_{H[\xi]} \not \equiv 0$

STATICITY THM

d.o.c. static

$\left[k_{[\alpha} \nabla_{\beta} k_{\gamma]}=0\right]$

STATICITY THM $\left(2^{\text {nd }}\right.$ part $)$

d.o.c. static and strictly stationary

$\left[k^{\mu} k_{\mu}<0\right]$

$\exists$ coordinate $t: k^{\mu}=\partial_{t}$ is hypersurface orthogonal

\section{STATIC UNIQUENESS THM}

[originally by means of Israel's thm, later by the positive energy thm]

Schwarzschild (Reissner-Nordström or MP)
STRONG RIGIDITY THM (2 ${ }^{\text {nd }}$ part $)$

d.o.c. axisymmetric

$\left[\exists\right.$ Killing field $\left.m^{\mu}\right]$

\section{CIRCULARITY THM}

d.o.c. circular

$\exists$ coordinates $t, \varphi: k^{\mu}=\partial_{t}$ and $m^{\mu}=\partial_{\varphi}$ are hypersurface orthogonal

\section{CIRCULAR UNIQUENESS THM}

[originally by means of Robinson's thm, later by $\sigma$-model/harmonic map identities or by inverse scattering techniques]

Kerr (Kerr-Newman) metric

Figure 3: Classification of analytic, connected, mean non-degenerate, asymptotically-flat, $I^{+}$-regular, stationary electrovacuum black holes. 
The hypotheses of analyticity and non-degeneracy are highly unsatisfactory, and one believes that they are not needed for the conclusion. One also believes that, in vacuum, the hypothesis of connectedness is spurious, and that all black holes with more than one component of the event horizon are singular, but no proof is available except for some special cases [216, 319, 249]. Indeed, Theorem 3.3 should be compared with the following conjecture, it being understood that both the Minkowski and the Reissner-Nordström spacetimes are members of the Kerr-Newman family:

CONJECTURE 3.4 Let $(M, \mathbf{g})$ be an electrovacuum, four-dimensional spacetime containing a spacelike, connected, acausal hypersurface $\mathscr{S}$, such that $\overline{\mathscr{S}}$ is a topological manifold with boundary, consisting of the union of a compact set and of a finite number of asymptotically-flat ends. Suppose that there exists on $M$ a complete stationary Killing vector $k$, that $\left\langle\left\langle M_{\mathrm{ext}}\right\rangle\right\rangle$ is globally hyperbolic, and that $\partial \overline{\mathscr{S}} \subset M \backslash\left\langle\left\langle M_{\text {ext }}\right\rangle\right\rangle$. Then $\left\langle\left\langle M_{\text {ext }}\right\rangle\right\rangle$ is isometric to the domain of outer communications of a Kerr-Newman or MP spacetime.

\subsubsection{A uniqueness theorem for near-Kerrian smooth vacuum stationary spacetimes}

The existing results on rigidity without analyticity require one to assume either staticity, or a near-Kerr condition on the spacetime geometry (see Section 3.3.1), which is quantified in terms of a smallness condition of the Mars-Simon tensor [223, 293]. The results in [3] together with Theorems 3.1-3.2, and a version of the Rácz-Wald Theorem [107, Proposition 4.1], lead to:

THEOREM 3.5 Let $(M, \mathbf{g})$ be a stationary asymptotically-flat, $I^{+}$-regular, smooth, vacuum fourdimensional spacetime. Assume that the event horizon is connected and mean non-degenerate. If the Mars-Simon tensor $S$ and the Ernst potential E of the spacetime satisfy

$$
\sum_{i j k l}\left|(1-\mathrm{E}) S_{i j k l}\right|<\epsilon
$$

for a small enough $\epsilon>0$, then $\left\langle\left\langle M_{\mathrm{ext}}\right\rangle\right\rangle$ is isometric to the domain of outer communications of a Kerr spacetime.

\subsection{Summary of open problems}

For the convenience of the reader, we summarize here the main open problems left in the no-hair theorem.

\subsubsection{Degenerate horizons}

We recall that there exist no vacuum static spacetimes containing degenerate horizons with compact spherical sections [81]. On the other hand, MP [220, 262] black holes provide the only electrovacuum static examples with non-connected degenerate horizons. See [78, 154] and references therein for a discussion of the geometry of MP black holes.

Under the remaining hypotheses of Theorem 3.3, the only step where the hypothesis of nondegeneracy enters is the proof of existence of a maximal hypersurface $\mathscr{S}$ in the black-hole spacetime under consideration, such that $\mathscr{S}$ is Cauchy for the domain of outer communications. The geometry of Cauchy surfaces in the case of degenerate horizons is well understood by now [62, 79], and has dramatically different properties when compared to the non-degenerate case. A proof of existence of maximal hypersurfaces in this case would solve the problem, but requires new insights. A key missing element is an equivalent of Bartnik's a priori height estimate [10], established for asymptotically-flat ends, that would apply to asymptotically-cylindrical ends. 
$\left(M\right.$, g) Stationary, asymptotically flat, electrovacuum, $I^{+}$-regular black-hole spacetime

[asymptotically time-like Killing field $k$; classification of isometry groups and actions]

\section{Regularity and topology theorems}

[horizons smooth/analytic with spherical sections; d.o.c. simply connected; structure theorem; nonexistence of prehorizons meeting the d.o.c.]

\section{STRONG RIGIDITY THM (1 ${ }^{\text {st }}$ part $)$}

Assume moreover that $(M, \mathbf{g})$ is either a) analytic, or b) vacuum and near-Kerrian with connected and mean non-degenerate event horizon $(\langle\kappa\rangle \neq 0)$.

Event horizon $=$ Killing horizon $H[\xi]$

$H[\xi]$ non-rotating: $\left.\mathbf{g}(k, k)\right|_{H(\xi)} \equiv 0$

$H[\xi]$ rotating: $\left.\mathbf{g}(k, k)\right|_{H(\xi)} \not \equiv 0$

If non-degenerate $(\kappa \neq 0)$ :

- Horizon essentially bifurcate

- Existence of maximal hypersurfaces

\section{STATICITY THM}

d.o.c. static

$d k^{b} \wedge k^{b}=0$
STRONG RIGIDITY THM ( ${ }^{\text {nd }}$ part $)$

d.o.c. axisymmetric

If $(M, \mathbf{g})$ analytic or near-Kerrian: $\exists$ periodic Killing field $m$ s.t. $[k, m]=0$ $M \approx \mathbb{R} \times\left(\mathbb{R}^{3} \backslash \cup_{i} B_{i}\right)$ $\rho^{2}:=\mathbf{g}^{2}(k, m)-\mathbf{g}(k, k) \mathbf{g}(m, m) \geq 0$ in d.o.c.

\section{CIRCULARITY THM}

STATICITY THM (2 ${ }^{\text {nd }}$ part $)$

d.o.c. static and strictly stationary

d.o.c. strictly stationary: $\mathbf{g}(k, k)<0$

$\exists$ coordinate $t: k=\partial_{t}$ is

hypersurface orthogonal

$\mathbf{g}=-V^{2} d t^{2}+\gamma_{i j} d x^{i} d x^{j}$

d.o.c. circular
$\exists$ global Weyl coordinates $(t, \varphi, \rho, z):$
$k=\partial_{t}, m=\partial_{\varphi}$
$\mathbf{g}=-\rho^{2} e^{2 \lambda} d t^{2}+e^{-2 \lambda}(d \varphi-v d t)^{2}+e^{2 u}\left(d \rho^{2}+d z^{2}\right)$
(with controlled asymptotic behavior)

\section{CIRCULAR UNIQUENESS THM}

- originally by means of Robinson's thm, later by $\sigma$-model/harmonic map identities or by inverse scattering techniques

- multi-black holes?

- No analyticity, degeneracy or connectedness needed

Kerr-Newman metric

Reissner-Nordström or MP

Figure 4: Classification of stationary electrovacuum black-hole spacetimes 


\subsubsection{Rigidity without analyticity}

Analyticity enters the current argument at two places: First, one needs to construct the second Killing vector near the horizon. This can be done by first constructing a candidate at the horizon, and then using analyticity to extend the candidate to a neighborhood of the horizon. Next, the Killing vector has to be extended to the whole domain of outer communications. This can be done using analyticity and a theorem by Nomizu [253], together with the fact that $I^{+}$-regular domains of outer communications are simply connected. Finally, analyticity can be used to provide a simple argument that prehorizons do not intersect $\left\langle\left\langle M_{\text {ext }}\right\rangle\right\rangle$ (but this is not critical, as a proof is available now within the smooth category of metrics [72]).

A partially-successful strategy to remove the analyticity condition has been invented by Alexakis, Ionescu and Klainerman in [2]. Their argument applies to non-degenerate near-Kerrian configurations, but the general case remains open.

The key to the approach in [2] is a unique continuation theorem near bifurcate Killing horizons proven in [174], which implies the existence of a second Killing vector field, say $m$, in a neighborhood of the horizon. One then needs to prove that $m$ extends to the whole domain of outer communications. This is established via another unique continuation theorem [175] with specific convexity conditions. These lead to non-trivial restrictions, and so far the argument has only been shown to apply to near-Kerrian configurations.

A unique continuation theorem across more general timelike surfaces would be needed to obtain the result without smallness restrictions.

It follows from what has been said in [72] that the boundary of the set where two Killing vector fields are defined cannot become null within a domain of outer communications; this fact might be helpful in solving the full problem.

\subsubsection{Many components?}

The only known examples of singularity-free stationary electrovacuum black holes with more than one component are provided by the MP family. (Axisymmetric MP solutions are possible, but MP metrics only have one Killing vector in general.) It has been suspected for a very long time that these are the only such solutions, and that there are thus no such vacuum configurations. This should be contrasted with the five-dimensional case, where the Black Saturn solutions of Elvang and Figueras [97] (compare [71, 305]) provide non-trivial two-component examples.

It might be convenient to summarize the general facts known about four-dimensional multicomponent solutions. ${ }^{4}$ In case of doubts, $I^{+}$-regularity should be assumed.

We start by noting that the static solutions, whether connected or not, have already been covered in Section 3.1.

A multi-component electro-vacuum configuration with all components non-degenerate and nonrotating would be, by what has been said, static, but then no such solutions exist (all components of an MP black hole are degenerate). On the other hand, the question of existence of a multiblack-hole configuration with components of mixed type, none of which rotates, is open; what's missing is the proof of existence of maximal hypersurfaces in such a case. Neither axisymmetry nor staticity is known for such configurations.

Analytic multi-black-hole solutions with at least one rotating component are necessarily axisymmetric; this leads one to study the corresponding harmonic-map equations, with candidate solutions provided by Weinstein or by inverse scattering techniques [198, 322, 23, 22]. The Weinstein solutions have no singularities away from the axes, but they are not known in

\footnotetext{
4 Here we are interested in stationary multi-black-hole configurations; nonexistence of some suitably regular stationary $n$-body configurations was established, under different symmetry conditions, in [20, 21].
} 
explicit form, which makes difficult the analysis of their behavior on the axis of rotation. The multi-black-hole metrics constructed by multi-soliton superpositions or by Bäcklund transformation techniques are obtained as rational functions with several parameters, with explicit constraints on the parameters that lead to a regular axis [222], but the analysis of the zeros of their denominators has proved intractable so far. It is perplexing that the five dimensional solutions, which are constructed by similar methods [268], can be completely analyzed with some effort and lead to regular solutions for some choices of parameters, but the four-dimensional case remains to be understood.

In any case, according to Varzugin [306, 307] and, independently, to Neugebauer and Meinel [251] (a more detailed exposition can be found in $[249,147]$ ), the multi-soliton solutions provide the only candidates for stationary axisymmetric electrovacuum solutions. A breakthrough in the understanding of vacuum two-component configurations has been made by Hennig and Neugebauer [147, 249], based on the area-angular momentum inequalities of Ansorg, Cederbaum and Hennig [145] as follows: Hennig and Neugebauer exclude many of the solutions by verifying that they have negative total ADM mass. Next, configurations where two horizons have vanishing surface gravity are shown to have zeros in the denominators of some geometric invariants. For the remaining ones, the authors impose a non-degeneracy condition introduced by Booth and Fairhurst [25]: a black hole is said to be sub-extremal if any neighborhood of the event horizon contains trapped surfaces. The key of the analysis is the angular momentum - area inequality of Hennig, Ansorg, and Cederbaum [145], that on every sub-extremal component of the horizon it holds that

$$
8 \pi|J|<A,
$$

where $J$ is the Komar angular-momentum and $A$ the area of a section. (It is shown in [7] and [146, Appendix] that $\kappa=0$ leads to equality in (3.1) under conditions relevant to the problem at hand.) Hennig and Neugebauer show that all remaining candidate solutions violate the inequality; this is their precise non-existence statement.

The problem with the argument so far is the lack of justification of the sub-extremality condition. Fortunately, this condition can be avoided altogether using ideas of [88] concerning the inequality (3.1) and appealing to the results in [96, 6, 73] concerning marginally-outer-trapped surfaces (MOTS): Using existence results of weakly stable MOTS together with various aspects of the candidate Weyl metrics, one can adapt the argument of [145] to show [70] that the area inequality (3.1), with "less than" there replaced by "less than or equal to", would hold for those components of the horizon, which have non-zero surface gravity, assuming an $I^{+}$-regular metric of the Weyl form, if any existed. The calculations of Hennig and Neugebauer [249] together with a contradiction argument lead then to

TheORem $3.6 I^{+}$-regular two-Kerr black holes do not exist.

The case of more than two horizons is widely open. 


\section{Classification of stationary toroidal Kaluza-Klein black holes}

In this work we are mostly interested in uniqueness results for four-dimensional black holes. This leads us naturally to consider those vacuum Kaluza-Klein spacetimes with enough symmetries to lead to four-dimensional spacetimes after dimensional reduction, providing henceforth fourdimensional black holes. It is convenient to start with a very short overview of the subject; the reader is referred to [101, 172] and references therein for more information. Standard examples of Kaluza-Klein black holes are provided by the Schwarzschild metric multiplied by any spatially flat homogeneous space (e.g., a torus). Non-trivial examples can be found in [272, 211]; see also [200, 172] and reference therein.

\subsection{Black holes in higher dimensions}

The study of spacetimes with dimension greater then four is almost as old as general relativity itself $[183,195]$. Concerning black holes, while in dimension four all explicitly-known asymptomaticallyflat and regular solutions of the vacuum Einstein equations are exhausted by the Kerr family, in spacetime dimension five the landscape of known solutions is richer. The following $I^{+}$-regular, stationary, asymptotically-flat, vacuum solutions are known in closed form: the Myers-Perry black holes, which are higher-dimensional generalizations of the Kerr metric with spherical-horizon topology [246]; the celebrated Emparan-Reall black rings with $S^{2} \times S^{1}$ horizon topology [99]; the Pomeransky-Senkov black rings generalizing the previous by allowing for a second angularmomentum parameter [269]; and the "Black Saturn" solutions discovered by Elvang and Figueras, which provide examples of regular multi-component black holes where a spherical horizon is surrounded by a black ring [97]. ${ }^{5}$

Inspection of the basic features of these solutions challenges any naive attempt to generalize the classification scheme developed for spacetime dimension four: One can find black rings and MyersPerry black holes with the same mass and angular momentum, which must necessarily fail to be isometric since the horizon topologies do not coincide. In fact there are non-isometric black rings with the same Poincaré charges; consequently a classification in terms of mass, angular momenta and horizon topology also fails. Moreover, the Black Saturns provide examples of regular vacuum multi-black-hole solutions, which are widely believed not to exist in dimension four; interestingly, there exist Black Saturns with vanishing total angular momentum, a feature that presumably distinguishes the Schwarzschild metric in four dimensions.

Nonetheless, results concerning 4-dimensional black holes either generalize or serve as inspiration in higher dimensions. This is true for landmark results concerning black-hole uniqueness and, in fact, classification schemes exist for classes of higher dimensional black-hole spacetimes, which mimic the symmetry properties of the "static or axisymmetric" alternative, upon which the uniqueness theory in four-dimensions is built.

For instance, staticity of $\mathrm{I}^{+}$-regular, vacuum, asymptotically-flat, non-rotating, non-degenerate black holes remains true in higher dimensions ${ }^{6}$. Also, Theorem 3.1 remains valid for vacuum spacetimes of dimension $n+1, n \geq 3$, whenever the positive energy theorem applies to an appropriate doubling of $\mathscr{S}$ (see [72], Section 3.1 and references therein). Moreover, the discussion in Section 3.1 together with the results in $[282,283]$ suggest that an analogous generalization to electrovacuum spacetimes exists, which would lead to uniqueness of the higher-dimensional Reissner-Nordström metrics within the class of static solutions of the Einstein-Maxwell equations, for all $n \geq 3$ (see also [101, Section 8.2], [173] and references therein).

\footnotetext{
5 Studies of regularity and causal structure of black rings and Saturns can be found in [82, 67, 68, 305, 71].

6 It should be noted that, although formulated for 4-dimensional spacetimes, the results in [84] remain valid without changes in higher-dimensional spacetimes.
} 
Rigidity theorems are also available for $(n+1)$-dimensional, asymptotically-flat and analytic black-hole spacetimes: the non-degenerate horizon case was established in [165] (compare [239]), and partial results concerning the degenerate case were obtained in [163]. These show that stationary rotating (analytic) black holes are "axisymmetric", in the sense that their isometry group contains $\mathbb{R} \times U(1)$; the $\mathbb{R}$ factor corresponds to the action generated by the stationary vector, while the circle action provides an "extra" axial Killing vector. A conjecture of Reall [274], supported by the results in [98], predicts the existence of 5-dimensional black holes with exactly $\mathbb{R} \times U(1)$ isometry group; in particular, it is conceivable that the rigidity results are sharp when providing only one "axial" Killing vector. The results in $[133,92,166]$ are likely to be relevant in this context.

So we see that, assuming analyticity and asymptotic flatness, the dichotomy provided by the rigidity theorem remains valid but its consequences appear to be weaker in higher dimensions. A gap appears between the two favorable situations encountered in dimension four: one being the already discussed staticity and the other corresponding to black holes with cohomogeneity-two Abelian groups of isometries. We will now consider this last scenario, which turns out to have connections to the four dimensional case.

\subsection{Stationary toroidal Kaluza-Klein black holes}

The four-dimensional vacuum Einstein equations simplify considerably in the stationary and axisymmetric setting by reducing to a harmonic map into the hyperbolic plane (see Sections 8 and 3.2.3). A similar such reduction in $(n+1)$-dimensions works when the isometry group includes $\mathbb{R} \times \mathbb{T}^{n-2}$, i.e., besides the stationary vector there exist $n-2$ commuting axial Killing vectors.

Since the center $\mathbb{T}^{s}$ of $S O(n)$ has dimension

$$
s=\left\lfloor\frac{n}{2}\right\rfloor,
$$

in the asymptotically flat case the existence of such a group of isometries is only possible for $n=3$ or $n=4$. However, one can move beyond the usual asymptotic-flatness and consider instead $K K$-asymptotically-flat spacetimes, in the sense of Section 2.2, with asymptotic ends $\mathscr{S}_{\text {ext }} \approx\left(\mathbb{R}^{N} \backslash B\right) \times \mathbb{T}^{s}$, satisfying $N=3,4$ and $N+s=n$, with the isometry group containing $\mathbb{R} \times \mathbb{T}^{n-2}, n \geq 3$. Here one takes $\mathscr{S}_{\text {ext }} \approx\left(\mathbb{R}^{N} \backslash \bar{B}(R)\right) \times \mathbb{T}^{s}$, with the reference metric of the form $\stackrel{\circ}{g}=\delta \oplus \stackrel{\circ}{h}$, where $\stackrel{h}{h}$ is the flat $s$-torus metric. Finally, the action of $\mathbb{R} \times \mathbb{T}^{n-2}$ on $(M, \mathbf{g})$ by isometries is assumed in the exterior region $M_{\text {ext }} \approx \mathbb{R} \times \mathscr{S}_{\text {ext }}$ to take the form

$$
\mathbb{R} \times \mathbb{T}^{n-2} \ni(\tau, g): \quad(t, p) \mapsto(t+\tau, g \cdot p) .
$$

Such metrics will be referred to as stationary toroidal Kaluza-Klein metrics.

\subsection{Topology of the event horizon}

A theorem of Galloway and Schoen [111] shows that compact cross-sections of the horizon must be of positive Yamabe type, i.e., admit metrics of positive scalar curvature. In spacetime dimension five, the positive Yamabe property restricts the horizon to be a finite connected sum of spaces with $S^{3}, S^{2} \times S^{1}$ and lens-space $L(p, q)$ topologies. Such results require no symmetry assumptions but by further assuming stationarity and the existence of one axial Killing vector more topological restrictions, concerning the allowed factors in the connected sum, appear in five dimensions [162].

In the toroidal Kaluza-Klein case, the existence of a toroidal action leads to further restrictions [169]; for instance, for $N=4$ and $n \geq 4$, each connected component of the horizon has necessarily one of the following topologies: $S^{3} \times \mathbb{T}^{n-4}, S^{2} \times T^{n-3}$ and $L(p, q) \times \mathbb{T}^{n-4}$. 
It should be noted that no asymptotically-flat or Kaluza-Klein black holes with lens-space topology of the horizon are known. Constructing a black lens, or establishing non-existence, appears to be a challenging problem.

\subsection{Orbit space structure}

The structure theorem [65] applies to stationary toroidal Kaluza-Klein black holes and provides the following product structure

$$
\left\langle\left\langle M_{\text {ext }}\right\rangle\right\rangle \approx \mathbb{R} \times \Sigma,
$$

with the stationary vector being tangent to the $\mathbb{R}$ factor and where $\Sigma$, endowed with the induced metric, is an $n$-dimensional Riemannian manifold admitting a $\mathbb{T}^{n-2}$ action by isometries. A careful analysis of the topological properties of such toroidal actions [169], based on deep results from [260, $261]$, allows one to show that the orbit space $\left\langle\left\langle M_{\text {ext }}\right\rangle\right\rangle /\left(\mathbb{R} \times \mathbb{T}^{n-2}\right)$ is homeomorphic to a half plane with boundary composed of segments and corners; the segments being the projection of either a component of the event horizon or an axis of rotation (the set of zeros of a linear combination of axial vectors), and the corners being the projections of the intersections of two axes. Moreover, the interiors are in fact diffeomorphic. To establish this last fundamental result it is necessary to exclude the existence of exceptional orbits of the toroidal action; this was done by Hollands and Yazadjiev in [169] by extending the results in [260] to the $K K$-black hole setting. In particular one obtains the following decomposition

$$
\left\langle\left\langle M_{\text {ext }}\right\rangle\right\rangle \backslash\left(\cup \mathscr{A}_{i}\right) \approx \mathbb{R} \times \mathbb{T}^{n-2} \times \mathbb{R} \times \mathbb{R}^{+},
$$

where $\cup \mathscr{A}_{i}$ is the union of all axes; we note that such product structure is necessary to the construction of Weyl coordinates $[76,65]$ and, consequently, indispensable to perform the desired reduction of the vacuum equations.

As already discussed, basic properties of black rings show that a classification of $K K$-black holes in terms of mass, angular momenta and horizon topology is not possible. But, as argued by Hollands and Yazadjiev [169], the angular momenta and the structure of the orbit space characterize such black holes if one further assumes non-degeneracy of the event horizon. This orbit space structure is in turn determined by the interval structure of the boundary of the quotient manifold, a concept related to Harmark's rod structure developed in [137] (see also [101, Section 5.2.2.1]). Note that the interval structure codifies the horizon topology.

\subsection{KK topological censorship}

Black-hole uniqueness in four-dimensions uses simple connectedness of the event horizon extensively. But the Schwarzschild metric multiplied by a flat torus shows that simple connectedness does not hold for general domains of outer communications of Kaluza-Klein black holes. Fortunately, simple connectedness of the orbit space $\left\langle\left\langle M_{\text {ext }}\right\rangle\right\rangle /\left(\mathbb{R} \times \mathbb{T}^{n-2}\right)$ suffices: for instance, to prove that the $(n-1)$-dimensional orbit generated by the stationary and axial vectors is timelike in $\left\langle\left\langle M_{\text {ext }}\right\rangle\right\rangle$ away from the axes (which in turn is essential to the construction of Weyl coordinates), to guarantee the existence of global twist potentials [65] and to exclude the existence of exceptional orbits of the toroidal action (see Section 4.4). The generalized topological censorship theorem of [73] shows that this property follows from the simple connectedness of the orbit space in the asymptotic end $\mathscr{S}_{\text {ext }} / \mathbb{T}^{n-2} \approx \mathbb{R}^{N} \backslash B$.

\subsection{Classification theorems for $K K$-black holes}

As usual, the static case requires separate consideration. The first classification results addressed static five-dimensional solutions with KK asymptotics and with a $\mathbb{R} \times U(1)$ factor in the group of 
isometries. In such a setting, the Kaluza-Klein reduction leads to gravity coupled with a Maxwell field $F$ and a "dilaton" field $\phi$, with a Lagrangean

$$
L=R-2(\nabla \phi)^{2}-e^{-2 \alpha \phi}|F|^{2},
$$

where $\alpha=\sqrt{3}$. In the literature one also considers more general theories where $\alpha$ does not necessarily take the Kaluza-Klein value. All current uniqueness proofs require that the mass, the Maxwell charges, and the dilaton charge satisfy a certain genericity condition, and that all horizon components have non-vanishing surface gravity. When $\alpha=1$, Mars and Simon [224] show that the generic static solutions belong to the family found by Gibbons and Maeda [131, 126, 122]. For other values of $\alpha$, in particular for the KK value, a purely electric or purely magnetic configuration is assumed, and then the same conclusion is reached. The result is an improvement on the original uniqueness theorems of Simon [294] and Masood-ul-Alam [226], and has been generalized to higher dimensions in [129]. The analyticity assumption, which is implicit in all the above proofs, can be removed using [72].

The remaining classfication results assume cohomogeneity-two isometry actions [169]:

TheOrem 4.1 Let $\left(M_{i}, \mathbf{g}_{i}\right), i=1,2$, be two $I^{+}$-regular, $(n+1)$-dimensional, $n \geq 3$, stationary toroidal Kaluza-Klein spacetimes, with five asymptotically-large dimensions $(N=4)$. Assume, moreover, that the event horizon is connected and mean non-degenerate. If the interval structure and the set of angular momenta coincide, then the domains of outer communications are isometric.

This theorem generalizes previous results by the same authors $[167,168]$ as well as a uniqueness result for a connected spherical black hole of [240].

The proof of Theorem 4.1 can be outlined as follows: After establishing the, mainly topological, results of Sections 4.3, 4.4 and 4.5, the proof follows closely the arguments for uniqueness of 4dimensional stationary and axisymmetric electrovacuum black holes. First, a generalized Mazur identity is valid in higher dimensions (see [218, 31] and Section 7.1). From this Hollands and Yazadjiev show that (compare the discussion in Sections 8.4.1 and 8.4.2)

$$
\Delta_{\delta} \psi \geq 0,
$$

where $\Delta_{\delta}$ is the flat Laplacian on $\mathbb{R}^{3}$, the function

$$
\psi: \mathbb{R}^{3} \backslash\{z=0\} \rightarrow \mathbb{R},
$$

is defined as

$$
\psi=\operatorname{Trace}\left(\Phi_{2} \Phi_{1}^{-1}-\mathbb{1}\right),
$$

the $\Phi_{i}$ 's, $i=1,2$, are the Mazur matrices [169, Eq. (78)] associated with the two black-hole spacetimes that are being compared. In terms of twist potentials $\left({ }^{(i)} \chi_{i}\right)$ and metric components of the axisymmetric Killing vectors (generators of the toroidal symmetry)

$$
{ }^{(i)} f_{m n}=\left({ }^{(i)} \mathbf{g}\left(k_{m}, k_{n}\right)\right), \quad i=1,2,
$$

we have the following explicit formula (see also [218, 240])

$$
\psi=-1+\frac{{ }^{(1)} f}{(2) f}+\frac{{ }^{(1)} f^{i j}\left({ }^{(1)} \chi_{i}-{ }^{(2)} \chi_{i}\right)\left({ }^{(1)} \chi_{j}-{ }^{(2)} \chi_{j}\right)}{{ }^{(2)} f}+{ }^{(1)} f^{i j}\left({ }^{(2)} f_{i j}-{ }^{(1)} f_{i j}\right),
$$

where ${ }^{(i)} f=\operatorname{det}\left({ }^{(i)} f_{m n}\right)$, and where ${ }^{(1)} f^{i j}$ is the matrix inverse to ${ }^{(1)} f_{i j}$.

It should be noted that this provides a variation on Mazur's and the harmonic map methods (see Sections 3.2.4 and 3.2.5), which avoids some of their intrinsic difficulties. Indeed, the integration 
by parts argument based on the Mazur identity requires detailed knowledge of the maps under consideration at the singular set $\{\rho=0\}$, while the harmonic map approach requires finding, and controlling, the distance function for the target manifold. (In some simple cases $\psi$ is the desired distance function, but whether this is so in general is unclear.) The result then follows by a careful analysis of the asymptotic behavior of the relevant fields; such analysis was also carried out in [169].

In this context, the degenerate horizons suffer from the supplementary difficulty of controlling the behavior of the fields near the horizon. One expects that an exhaustive analysis of near-horizon geometries would allow one to settle the question; some partial results towards this can be found in $[204,203,202,164]$. 


\section{Beyond Einstein-Maxwell}

The purpose of this section is to reexamine the various steps leading to the classification of electrovacuum black-hole spacetimes for other matter models. In particular, it will be seen that several steps in Figure 3 cease to hold in the presence of non-Abelian gauge fields. Unfortunately, this implies that we are far from having a classification of all stationary black-hole spacetimes with physically-interesting sources.

\subsection{Spherically symmetric black holes with hair}

One can find in the literature the naive expectation that - within a given matter model - the stationary black-hole solutions are uniquely characterized by a set of global charges; this will be referred to as the generalized no-hair conjecture. A model in which this might possibly be correct is provided by the static sector of the EM-dilaton theory, discussed at the beginning of Section 4.6.

The failure of this generalized no-hair conjecture is demonstrated by the Einstein-Yang-Mills (EYM) theory: According to the conjecture, any static solution of the EYM equations should either coincide with the Schwarzschild metric or have some non-vanishing Yang-Mills charges. This turned out not to be the case when, in 1989, various authors [310, 208, 24] found a family of static black-hole solutions with vanishing global Yang-Mills charges (as defined, e.g., in [74]); these were originally constructed by numerical means and rigorous existence proofs were given later in [299, 297, 298, 29, 227]; for a review see [311]. These solutions violate the generalized no-hair conjecture.

As the non-Abelian black holes are unstable [301, 329, 315], one might adopt the view that they do not present actual threats to the generalized no-hair conjecture. (The reader is referred to [37] for the general structure of the pulsation equations, [309, 40], to [27] for the sphaleron instabilities of the particle-like solutions, and to [292] for a review on sphalerons.) However, various authors have found stable black holes, which are not characterized by a set of asymptotic flux integrals. For instance, there exist stable black-hole solutions with hair of the static, spherically-symmetric Einstein-Skyrme equations [94, 156, 157, 161, 241] and to the EYM equations coupled to a Higgs triplet $[28,30,214,1]$; it should be noted that the solutions of the EYM-Higgs equations with a Higgs doublet are unstable [27, 324]. Hence, the restriction of the generalized no-hair conjecture to stable configurations is not correct either.

One of the reasons why it was not until 1989 that black-hole solutions with self-gravitating gauge fields were discovered was the widespread belief that the EYM equations admit no soliton solutions. There were, at least, five reasons in support of this hypothesis.

- First, there exist no purely gravitational solitons, that is, the only globally-regular, asymptotically-flat, static vacuum solution to the Einstein equations with finite energy is Minkowski spacetime. This is the Lichnerowicz theorem, which nowadays can be obtained from the positive mass theorem and the Komar expression for the total mass of an asymptoticallyflat, stationary spacetime [170]; see, e.g., [127] or [152]. A rather strong version thereof, which does not require asymptotic conditions other than completeness of the space metric, has been established by Anderson [5], see also [4].

- Next, there are no nontrivial static solutions of the EYM equations near Minkowski spacetime [221].

- Further, both Deser's energy argument [90] and Coleman's scaling method [87] show that there do not exist pure YM solitons in flat spacetime.

- Moreover, the EM system admits no soliton solutions. (This follows by applying Stokes' theorem to the static Maxwell equations; see, e.g., [151].) 
- Finally, Deser [91] proved that the three-dimensional EYM equations admit no soliton solutions. The argument takes advantage of the fact that the magnetic part of the Yang-Mills field has only one non-vanishing component in $2+1$ dimensions.

All this shows that it was conceivable to conjecture a nonexistence theorem for soliton solutions of the EYM equations in $3+1$ dimensions, and a no-hair theorem for the corresponding black hole configurations. On the other hand, none of the above examples takes care of the full nonlinear EYM system, which bears the possibility to balance the gravitational and the gauge field interactions. In fact, a closer look at the structure of the EYM action in the presence of a Killing symmetry dashes the hope to generalize the uniqueness proof along the lines used in the Abelian case: The Mazur identity owes its existence to the $\sigma$-model formulation of the EM equations. The latter is, in turn, based on scalar magnetic potentials, the existence of which is a peculiarity of Abelian gauge fields (see Section 6).

\subsection{Static black holes without spherical symmetry}

The above counterexamples to the generalized no-hair conjecture are static and spherically symmetric. The famous Israel theorem guarantees that spherical symmetry is, in fact, a consequence of staticity, provided that one is dealing with vacuum [176] or electrovacuum [177] black-hole spacetimes. The task to extend the Israel theorem to more general self-gravitating matter models is, of course, a difficult one. In fact, the following example proves that spherical symmetry is not a generic property of static black holes.

In [213], Lee et al. reanalyzed the stability of the Reissner-Nordström (RN) solution in the context of $S U(2)$ EYM-Higgs theory. It turned out that - for sufficiently small horizons - the RN black holes develop an instability against radial perturbations of the Yang-Mills field. This suggested the existence of magnetically-charged, spherically-symmetric black holes with hair, which were also found by numerical means $[28,30,214,1]$.

Motivated by these solutions, Ridgway and Weinberg [277] considered the stability of the magnetically charged RN black holes within a related model; the EM system coupled to a charged, massive vector field. Again, the RN solution turned out to be unstable with respect to fluctuations of the massive vector field. However, a perturbation analysis in terms of spherical harmonics revealed that the fluctuations cannot be radial (unless the magnetic charge assumes an integer value), as discussed in Weinberg's comprehensive review on magnetically-charged black holes [317]. In fact, the work of Ridgway and Weinberg shows that static black holes with magnetic charge need not even be axially symmetric [278]. Axisymmetric, static black holes without spherical symmetry appear to exist within the pure EYM system and the EYM-dilaton model [194].

This shows that static black holes may have considerably more structure than one might expect from the experience with the EM system: Depending on the matter model, they may allow for nontrivial fields outside the horizon and, moreover, they need not be spherically symmetric. Even more surprisingly, there exist static black holes without any rotational symmetry at all.

\subsection{The Birkhoff theorem}

The Birkhoff theorem shows that the domain of outer communication of a spherically-symmetric black-hole solution to the vacuum or the EM equations is static. The result does not apply to many other matter models: dust, fluids, scalar fields, Einstein-Vlasov, etc., and it is natural to raise the question for non-Abelian gauge fields. Now, the Einstein Yang-Mills equations have a well-posed Cauchy problem, so one needs to make sure that the constraint equations admit non-stationary solutions: Bartnik [11] has indeed proved existence of such initial data. The problem has also been addressed numerically in [328, 329], where spherically-symmetric solutions of the EYM equations describing the explosion of a gauge boson star or its collapse to a Schwarzschild black hole have 
been found. A systematic study of the problem for the EYM system with arbitrary gauge groups was performed by Brodbeck and Straumann [39]. Extending previous results of Künzle [205] (see also [206, 207]), the authors of [39] were able to classify the principal bundles over spacetime, which - for a given gauge group - admit $S O(3)$ as symmetry group, acting by bundle automorphisms. It turns out that the Birkhoff theorem can be generalized to bundles, which admit only $S O(3)$ invariant connections of Abelian type. We refer to [39] for the precise formulation of the statement in terms of Stiefel diagrams, and to [33, 34, 138, 255] for a classification of EYM solitons. The results in $[104,13]$ concerning particle-like EYM solutions are likely to be relevant for the corresponding black-hole problem, but no detailed studies of this exist so far.

\subsection{The staticity problem}

Going back one step further in the left half of the classification scheme displayed in Figure 3, one is led to the question of whether all black holes with non-rotating horizon are static. For non-degenerate EM black holes this issue was settled by Sudarsky and Wald [302, 303, 84], ${ }^{7}$ while the corresponding vacuum problem was solved quite some time ago [143]; the degenerate case remains open. Using a slightly improved version of the argument given in [143], the staticity theorem can be generalized to self-gravitating stationary scalar fields and scalar mappings [152] as, for instance, the Einstein-Skyrme system. (See also [158, 149, 160], for more information on the staticity problem). It should also be noted that the proof given in [152] works under less restrictive topological assumptions, since it does not require the global existence of a twist potential.

While the vacuum and the scalar staticity theorems are based on differential identities and integration by parts, the approach due to Sudarsky and Wald takes advantage of the ADM formalism and the existence of a maximal slicing [84]. Along these lines, the authors of [302, 303] were able to extend the staticity theorem to topologically-trivial non-Abelian black-hole solutions. However, in contrast to the Abelian case, the non-Abelian version applies only to configurations for which either all components of the electric Yang-Mills charge or the electric potential vanish asymptotically. This leaves some room for stationary black holes, which are non-rotating and not static. Moreover, the theorem implies that such configurations must be charged. On a perturbative level, the existence of these charged, non-static black holes with vanishing total angular momentum was established in [38].

\subsection{Rotating black holes with hair}

So far we have addressed the ramifications occurring on the "non-rotating half" of the classification diagram of Figure 3: We have argued that non-rotating black holes need not be static; static ones need not be spherically symmetric; and spherically-symmetric ones need not be characterized by a set of global charges. The right-hand-side of the classification scheme has been studied less intensively so far. Here, the obvious questions are the following: Are all stationary black holes with rotating Killing horizons axisymmetric (rigidity)? Are the stationary and axisymmetric Killing fields orthogonally-transitive (circularity)? Are the circular black holes characterized by their mass, angular momentum and global charges (no-hair)?

Let us start with the first issue, concerning the generality of the strong rigidity theorem (SRT). The existence of a second Killing vector field to the future of a bifurcation surface can be established by solving a characteristic Cauchy problem [107], which makes it clear that axial symmetry will hold for a large class of matter models satisfying the, say, dominant energy condition.

The counterpart to the staticity problem is the circularity problem: As general non-rotating black holes are not static, one expects that the axisymmetric ones need not be circular. This is,

\footnotetext{
7 An early apparent success rested on a sign error [46]. Carter's amended version of the proof was subject to a certain inequality between the electric and the gravitational potential [50].
} 
indeed, the case: While circularity is a consequence of the EM equations and the symmetry properties of the electro-magnetic field, the same is not true for the EYM system. In the Abelian case, the proof rests on the fact that the field tensor satisfies $F(k, m)=(* F)(k, m)=0, k$ and $m$ being the stationary and the axial Killing field, respectively; for Yang-Mills fields these conditions do no longer follow from the field equations and their invariance properties (see Section 8.1 for details). Hence, the familiar Papapetrou ansatz for a stationary and axisymmetric metric is too restrictive to take care of all stationary and axisymmetric degrees of freedom of the EYM system. However, there are other matter models for which the Papapetrou metric is sufficiently general: the proof of the circularity theorem for self-gravitating scalar fields is, for instance, straightforward [150]. Recalling the key simplifications of the EM equations arising from the (2+2)-splitting of the metric in the Abelian case, an investigation of non-circular EYM equations is expected to be rather awkward. As rotating black holes with hair are most likely to occur already in the circular sector (see the next paragraph), a systematic investigation of the EYM equations with circular constraints is needed as well.

The static subclass of the circular sector was investigated in studies by Kleihaus and Kunz (see [194] for a compilation of the results). Since, in general, staticity does not imply spherical symmetry, there is a possibility for a static branch of axisymmetric black holes without spherical symmetry. Using numerical methods, Kleihaus and Kunz have constructed black-hole solutions of this kind for both the EYM and the EYM-dilaton system [192]. The related axisymmetric soliton solutions without spherical symmetry were previously obtained by the same authors [190, 191]; see also [193] for more details. The new configurations are purely magnetic and parameterized by their winding number and the node number of the relevant gauge field amplitude. In the formal limit of infinite node number, the EYM black holes approach the Reissner-Nordström solution, while the EYM-dilaton black holes tend to the Gibbons-Maeda black hole [126, 131]. The solutions themselves are neutral and not spherically symmetric; however, their limiting configurations are charged and spherically symmetric. Both the soliton and the black-hole solutions of Kleihaus and Kunz are unstable and may, therefore, be regarded as gravitating sphalerons and black holes inside sphalerons, respectively.

Existence of slowly rotating regular black-hole solutions to the EYM equations was established in [38]. Using the reduction of the EYM action in the presence of a stationary symmetry reveals that the perturbations giving rise to non-vanishing angular momentum are governed by a self-adjoint system of equations for a set of gauge invariant fluctuations [35]. With a soliton background, the solutions to the perturbation equations describe charged, rotating excitations of the BartnikMcKinnon solitons [14]. In the black-hole case the excitations are combinations of two branches of stationary perturbations: The first branch comprises charged black holes with vanishing angular momentum, ${ }^{8}$ whereas the second one consists of neutral black holes with non-vanishing angular momentum. (A particular combination of the charged and the rotating branch was found in [312].) In the presence of bosonic matter, such as Higgs fields, the slowly rotating solitons cease to exist, and the two branches of black-hole excitations merge to a single one with a prescribed relation between charge and angular momentum [35]. More information about the EYM-Higgs system can be found in [209, 254].

\footnotetext{
8 As already mentioned in Section 5.4, these black holes present counter-examples to the naive generalization of the staticity theorem; they are nice illustrations of the correct non-Abelian version of the theorem [302, 303].
} 


\section{$6 \quad$ Stationary Spacetimes}

For physical reasons, the black-hole equilibrium states are expected to be stationary. Spacetimes admitting a Killing symmetry exhibit a variety of interesting features, some of which will be discussed in this section. In particular, the existence of a Killing field implies a canonical local 3+1 decomposition of the metric. The projection formalism arising from this structure was developed by Geroch in the early seventies [125, 124], and can be found in Chapter 16 of the book on exact solutions by Kramer et al. [199].

A slightly different, rather powerful approach to stationary spacetimes is obtained by taking advantage of their Kaluza-Klein (KK) structure. As this approach is less commonly used in the present context, we will discuss the KK reduction of the Einstein-Hilbert(-Maxwell) action in some detail, the more so as this yields an efficient derivation of the Ernst equations and the Mazur identity. Moreover, the inclusion of non-Abelian gauge fields within this framework [35] reveals a decisive structural difference between the Einstein-Maxwell (EM) and the Einstein-Yang-Mills (EYM) system.

\subsection{Reduction of the Einstein-Hilbert action}

By definition, a stationary spacetime $(M, \mathbf{g})$ admits an asymptotically-time-like Killing field, that is, a vector field $k$ with $L_{k} \mathbf{g}=0, L_{k}$ denoting the Lie derivative with respect to $k$. At least locally, $M$ has the structure $\Sigma \times G$, where $G \approx \mathbb{R}$ denotes the one-dimensional group generated by the Killing symmetry, and $\Sigma$ is the three-dimensional quotient space $M / G$. A stationary spacetime is called static, if the integral trajectories of $k$ are orthogonal to $\Sigma$.

With respect to an adapted coordinate $t$, so that $k:=\partial_{t}$, the metric of a stationary spacetime can be parameterized in terms of a three-dimensional (Riemannian) metric $\overline{\boldsymbol{g}}:=\bar{g}_{i j} \mathrm{~d} x^{i} \mathrm{~d} x^{j}$, a oneform $a:=a_{i} \mathrm{~d} x^{i}$, and a scalar field $V$, where stationarity implies that $\bar{g}_{i j}, a_{i}$ and $V$ are functions on $(\Sigma, \bar{g})$ :

$$
\mathbf{g}=-V(\mathrm{~d} t+a)^{2}+\frac{1}{V} \overline{\boldsymbol{g}}
$$

The notation $t$ suggests that $t$ is a time coordinate, $\mathbf{g}(\nabla t, \nabla t)<0$, but this restriction does not play any role in the local form of the equations that we are about to derive. Similarly the local calculations that follow remain valid regardless of the causal character of $k$, provided that $k$ is not null everywhere, and then one only considers the region where $\mathbf{g}(k, k) \equiv-V$ does not change sign. On any connected component of this region $k$ is either spacelike or timelike, as determined by the sign of $V$, and then the metric $\overline{\boldsymbol{g}}$ is Lorentzian, respectively Riemannian, there. In any case, both the parameterization of the metric and the equations become singular at places where $V$ has zeros, so special care is required wherever this occurs.

Using Cartan's structure equations (see, e.g., [300]), it is a straightforward task to compute the Ricci scalar for the above decomposition of the spacetime metric; see, e.g., [155] for the details of the derivation. The result is that the Einstein-Hilbert action of a stationary spacetime reduces to the action for a scalar field $V$ and a vector field $a$, which are coupled to three-dimensional gravity. The fact that this coupling is minimal is a consequence of the particular choice of the conformal factor in front of the three-metric $\overline{\boldsymbol{g}}$ in the decomposition (6.1). The vacuum field equations are thus seen to be equivalent to the three-dimensional Einstein-matter equations obtained from variations of the effective action

$$
S_{\text {eff }}=\int \bar{*}\left(\bar{R}-\frac{1}{2 V^{2}}\langle\mathrm{~d} V, \mathrm{~d} V\rangle+\frac{V^{2}}{2}\langle\mathrm{~d} a, \mathrm{~d} a\rangle\right),
$$

with respect to $\bar{g}_{i j}, V$ and $a$. Here and in the following $\bar{R}$ denotes the Ricci scalar of $\overline{\boldsymbol{g}}$, while for $p$-forms $\alpha$ and $\beta$, their inner product is defined by $\bar{*}\langle\alpha, \beta\rangle:=\alpha \wedge \bar{*} \beta$, where $\bar{*}$ is the Hodge dual with respect to $\overline{\boldsymbol{g}}$. 
It is worth noting that the quantities $V$ and $a$ are related to the norm and the twist of the Killing field as follows:

$$
V=-\mathbf{g}(k, k), \quad \omega:=\frac{1}{2} *(k \wedge \mathrm{d} k)=-\frac{1}{2} V^{2} \bar{*} \mathrm{~d} a,
$$

where $*$ and $\bar{*}$ denote the Hodge dual with respect to $\mathbf{g}$ and $\overline{\boldsymbol{g}}$, respectively. Here and in the following we use the symbol $k$ for both the Killing field $\partial_{t}$ and the corresponding one-form $-V(\mathrm{~d} t+a)$. One can view $a$ as a connection on a principal bundle with base space $\Sigma$ and fiber $\mathbb{R}$, since it behaves like an Abelian gauge potential under coordinate transformations of the form $t \rightarrow t+\varphi\left(x^{i}\right)$. Not surprisingly, it enters the effective action in a gauge-invariant way, that is, only via the "Abelian field strength", $f:=\mathrm{d} a$.

\subsection{The coset structure of vacuum gravity}

For many applications, in particular for the black-hole uniqueness theorems, it is convenient to replace the one-form $a$ by a function, namely the twist potential. We have already pointed out that $a$, parameterizing the non-static part of the metric, enters the effective action (6.2) only via the field strength, $f=\mathrm{d} a$. For this reason, the variational equation for $a$ (that is, the off-diagonal Einstein equation) takes in vacuum the form of a source-free Maxwell equation:

$$
\mathrm{d} \bar{*}\left(V^{2} \mathrm{~d} a\right)=0 \Longrightarrow \mathrm{d} Y=-\bar{*}\left(V^{2} \mathrm{~d} a\right) .
$$

By virtue of Eq. (6.3), the (locally-defined) function $Y$ is a potential for the twist one-form, $\mathrm{d} Y=2 \omega$. In order to write the effective action (6.2) in terms of the twist potential $Y$, rather than the one-form $a$, one considers $f$ as a fundamental field and imposes the constraint $\mathrm{d} f=0$ with the Lagrange multiplier $Y$. The variational equation with respect to $f$ then yields $f=-\bar{*}\left(V^{-2} \mathrm{~d} Y\right)$, which is used to eliminate $f$ in favor of $Y$. One finds $\frac{1}{2} V^{2} f \wedge \bar{*} f-Y \mathrm{~d} f \rightarrow-\frac{1}{2} V^{-2} \mathrm{~d} Y \wedge \bar{*} \mathrm{~d} Y$. Thus, the action (6.2) becomes

$$
S_{\text {eff }}=\int \bar{*}\left(\bar{R}-\frac{\langle\mathrm{d} V, \mathrm{~d} V\rangle+\langle\mathrm{d} Y, \mathrm{~d} Y\rangle}{2 V^{2}}\right),
$$

where we recall that $\langle$,$\rangle is the inner product with respect to the three-metric \overline{\boldsymbol{g}}$ defined in Eq. (6.1).

The action (6.5) describes a harmonic map into a two-dimensional target space, effectively coupled to three-dimensional gravity. In terms of the complex Ernst potential E [102, 103], one has

$$
S_{\mathrm{eff}}=\int \bar{*}\left(\bar{R}-2 \frac{\langle\mathrm{dE}, \mathrm{d} \overline{\mathrm{E}}\rangle}{(\mathrm{E}+\overline{\mathrm{E}})^{2}}\right), \quad \mathrm{E}:=V+i Y
$$

The stationary vacuum equations are obtained from variations with respect to the three-metric $\overline{\boldsymbol{g}}$ $[(i j)$-equations $]$ and the Ernst potential $\mathrm{E}[(0 \mu)$-equations $]$. One easily finds $\bar{R}_{i j}=2(\mathrm{E}+\overline{\mathrm{E}})^{-2} \mathrm{E},{ }_{i} \overline{\mathrm{E}},_{j}$ and $\bar{\Delta} \mathrm{E}=2(\mathrm{E}+\overline{\mathrm{E}})^{-1}\langle\mathrm{dE}, \mathrm{dE}\rangle$, where $\bar{\Delta}$ is the Laplacian with respect to $\overline{\boldsymbol{g}}$.

The target space for stationary vacuum gravity, parameterized by the Ernst potential E, is a Kähler manifold with metric $G_{\mathrm{EE}}=\partial_{\mathrm{E}} \partial_{\overline{\mathrm{E}}} \ln (V)$ (see [115] for details). By virtue of the mapping

$$
\mathrm{E} \mapsto z=\frac{1-\mathrm{E}}{1+\mathrm{E}}
$$

the semi-plane where the Killing field is time-like, $\operatorname{Re}(\mathrm{E})>0$, is mapped into the interior of the complex unit disc, $D=\{z \in \mathbb{C}|| z \mid<1\}$, with standard metric $\left(1-|z|^{2}\right)^{-2}\langle\mathrm{~d} z, \mathrm{~d} \bar{z}\rangle$. By virtue of the stereographic projection, $\operatorname{Re}(z)=x^{1}\left(x^{0}+1\right)^{-1}, \operatorname{Im}(z)=x^{2}\left(x^{0}+1\right)^{-1}$, the unit disc $D$ is isometric to the pseudo-sphere, $P S^{2}=\left\{\left(x^{0}, x^{1}, x^{2}\right) \in \mathbb{R}^{3} \mid-\left(x^{0}\right)^{2}+\left(x^{1}\right)^{2}+\left(x^{2}\right)^{2}=-1\right\}$. As the 
three-dimensional Lorentz group, $S O(2,1)$, acts transitively and isometrically on the pseudo-sphere with isotropy group $S O(2)$, the target space is the coset $P S^{2} \approx S O(2,1) / S O(2)$ (see, e.g., [196] or [26] for the general theory of symmetric spaces). Using the universal covering $S U(1,1)$ of $S O(2,1)$, one can parameterize $P S^{2} \approx S U(1,1) / U(1)$ in terms of a positive hermitian matrix $\Phi(x)$, defined by

$$
\Phi(x)=\left(\begin{array}{cc}
x^{0} & x^{1}+i x^{2} \\
x^{1}-i x^{2} & x^{0}
\end{array}\right)=\frac{1}{1-|z|^{2}}\left(\begin{array}{cc}
1+|z|^{2} & 2 z \\
2 \bar{z} & 1+|z|^{2}
\end{array}\right) .
$$

Hence, the effective action for stationary vacuum gravity becomes the standard action for a $\sigma$-model coupled to three-dimensional gravity [250],

$$
\mathcal{S}_{\text {eff }}=\int \bar{*}\left(\bar{R}-\frac{1}{4} \operatorname{Trace}\langle\mathcal{J}, \mathcal{J}\rangle\right),
$$

where

$$
\operatorname{Trace}\langle\mathcal{J}, \mathcal{J}\rangle \equiv\left\langle\mathcal{J}_{B}^{A}, \mathcal{J}_{A}^{B}\right\rangle:=\bar{g}^{i j}\left(\mathcal{J}_{i}\right)_{B}^{A}\left(\mathcal{J}_{j}\right)_{A}^{B},
$$

and the currents $\mathcal{J}_{i}$ are defined as

$$
\mathcal{J}_{i}:=\Phi^{-1} \bar{\nabla}_{i} \Phi
$$

The simplest nontrivial solution to the vacuum Einstein equations is obtained in the static, spherically-symmetric case: For $\mathrm{E}=V(r)$ one has $2 \bar{R}_{r r}=\left(V^{\prime} / V\right)^{2}$ and $\bar{\Delta} \ln (V)=0$. With respect to the general spherically-symmetric ansatz

$$
\overline{\boldsymbol{g}}=\mathrm{d} r^{2}+\rho^{2}(r) \mathrm{d} \Omega^{2},
$$

one immediately obtains the equations $-4 \rho^{\prime \prime} / \rho=\left(V^{\prime} / V\right)^{2}$ and $\left(\rho^{2} V^{\prime} / V\right)^{\prime}=0$, the solution of which is the Schwarzschild metric in the usual parametrization: $V=1-2 M / r, \rho^{2}=V(r) r^{2}$.

\subsection{Stationary gauge fields}

The reduction of the Einstein-Hilbert action in the presence of a Killing field yields a $\sigma$-model, which is effectively coupled to three-dimensional gravity. While this structure is retained for the EM system, it ceases to exist for self-gravitating non-Abelian gauge fields. In order to perform the dimensional reduction for the EM and the EYM equations, we need to recall the notion of a symmetric gauge field.

In mathematical terms, a gauge field (with gauge group $G$, say) is a connection in a principal bundle $P(M, G)$ over spacetime $M$. A gauge field is symmetric with respect to the action of a symmetry group $S$ of $M$, if it is described by an $S$-invariant connection on $P(M, G)$. Hence, finding the symmetric gauge fields involves the task of classifying the principal bundles $P(M, G)$, which admit the symmetry group $S$, acting by bundle automorphisms. This program was carried out by Brodbeck and Straumann for arbitrary gauge and symmetry groups [33], (see also [34, 39]), generalizing earlier work of Harnad et al. [138], Jadczyk [181] and Künzle [207].

The gauge fields constructed in the above way are invariant under the action of $S$ up to gauge transformations. This is also the starting point of the alternative approach to the problem, due to Forgács and Manton [105]. It implies that a gauge potential $A$ is symmetric with respect to the action of a Killing field $\xi$, say, if there exists a Lie algebra valued function $\mathcal{V}_{\xi}$, such that

$$
L_{\xi} A=\mathrm{D} \mathcal{V}_{\xi},
$$

where $\mathcal{V}_{\xi}$ is the generator of an infinitesimal gauge transformation, $L_{\xi}$ denotes the Lie derivative, and $\mathrm{D}$ is the gauge covariant exterior derivative, $\mathrm{D} \mathcal{V}_{\xi}=\mathrm{d} \mathcal{V}_{\xi}+\left[A, \mathcal{V}_{\xi}\right]$ 
Let us now consider a stationary spacetime with (asymptotically) time-like Killing field $k$. A stationary gauge potential can be parameterized in terms of a one-form $\bar{A}$ orthogonal to $k$, in the sense that $\bar{A}(k)=0$, and a Lie algebra valued potential $\phi$,

$$
A=\phi(\mathrm{d} t+a)+\bar{A}
$$

where we recall that $a$ is the non-static part of the metric (6.1). For the sake of simplicity we adopt a gauge where $\mathcal{V}_{k}$ vanishes. ${ }^{9}$ By virtue of the above decomposition, the field strength becomes $F=\overline{\mathrm{D}} \phi \wedge(\mathrm{d} t+a)+(\bar{F}+\phi f)$, where $\bar{F}$ is the Yang-Mills field strength for $\bar{A}$ and $f=d a$. Using the expression (6.5) for the vacuum action, one easily finds that the EYM action,

$$
S_{\mathrm{EYM}}=\int(* R-2 \hat{\operatorname{tr}}(F \wedge * F))
$$

where $R$ and $*$ refer to the 4-dimensional space-time metric $\mathbf{g}$ and $\hat{\operatorname{tr}}()$ denotes a suitably normalized trace (e.g., $\hat{\operatorname{tr}}\left(\tau_{a} \tau_{b}\right)=\frac{1}{2} \delta_{a b}$ where the $\sigma_{a}$ 's are the Pauli matrices), gives rise to the effective action

$$
S_{\text {eff }}=\int \bar{*}\left(\bar{R}-\frac{1}{2 V^{2}}|\mathrm{~d} V|^{2}+\frac{V^{2}}{2}|f|^{2}+\frac{2}{V}|\overline{\mathrm{D}} \phi|^{2}-2 V|\bar{F}+\phi f|^{2}\right),
$$

where $\overline{\mathrm{D}}$ is the gauge covariant derivative with respect to $\bar{A}$, and where the inner product also involves the trace: $\bar{*}|\bar{F}|^{2}:=\hat{\operatorname{tr}}(\bar{F} \wedge \bar{*} \bar{F})$. The above action describes two scalar fields, $V$ and $\phi$, and two vector fields, $a$ and $\bar{A}$, which are minimally coupled to three-dimensional gravity with metric $\overline{\boldsymbol{g}}$. Similarly to the vacuum case, the connection $a$ enters $S_{\text {eff }}$ only via the field strength $f$. Again, this gives rise to a differential conservation law,

$$
\mathrm{d} \bar{*}\left[V^{2} f-4 V \hat{\operatorname{tr}}(\phi(\bar{F}+\phi f))\right]=0
$$

by virtue of which one can (locally) introduce a generalized twist potential $Y$, defined by $-\mathrm{d} Y=$ $\bar{*}[\ldots]$.

The main difference between the Abelian and the non-Abelian case concerns the variational equation for $\bar{A}$, that is, the Yang-Mills equation for $\bar{F}$ : For non-Abelian gauge groups, $\bar{F}$ is no longer an exact two-form, and the gauge covariant derivative of $\phi$ introduces source terms in the corresponding Yang-Mills equation:

$$
\overline{\mathrm{D}}[V \bar{*}(\bar{F}+\phi f)]=V^{-1} \bar{*}[\phi, \overline{\mathrm{D}} \phi] .
$$

Hence, the scalar magnetic potential - which can be introduced in the Abelian case according to $d \psi:=V \bar{*}(\bar{F}+\phi f)-$ ceases to exist for non-Abelian Yang-Mills fields. The remaining stationary EYM equations are easily derived from variations of $S_{\text {eff }}$ with respect to the gravitational potential $V$, the electric Yang-Mills potential $\phi$ and the three-metric $\overline{\boldsymbol{g}}$.

As an application, we note that the effective action (6.16) is particularly suited for analyzing stationary perturbations of static $(a=0)$, purely magnetic $(\phi=0)$ configurations [35], such as the Bartnik-McKinnon solitons [14] and the corresponding black-hole solutions [310, 208, 24]. The two crucial observations in this context are [35, 312]:

(i) The only perturbations of the static, purely magnetic EYM solutions, which can contribute the ADM angular momentum are the purely non-static, purely electric ones, $\delta a$ and $\delta \phi$.

(ii) In first-order perturbation theory, the relevant fluctuations, $\delta a$ and $\delta \phi$, decouple from the remaining metric and matter perturbations.

9 The symmetry condition (6.13) translates into $L_{k} \phi=[\phi, \mathcal{V}]$ and $L_{k} \bar{A}=\overline{\mathrm{D}} \mathcal{V}$, which can be used to reduce the EYM equations in the presence of a Killing symmetry in a gauge-invariant manner [158, 159]. 
The second observation follows from the fact that the magnetic Yang-Mills equation (6.18) and the Einstein equations for $V$ and $\overline{\boldsymbol{g}}$ become background equations, since they contain no linear terms in $\delta a$ and $\delta \phi$. Therefore, the purely electric, non-static perturbations are governed by the twist equation (6.17) and the electric Yang-Mills equation (obtained from variations of $S_{\text {eff }}$ with respect to $\phi)$.

Using Eq. (6.17) to introduce the twist potential $Y$, the fluctuation equations for the first-order quantities $\delta Y$ and $\delta \phi$ assume the form of a self-adjoint system [35]. Considering perturbations of spherically-symmetric configurations, one can expand $\delta Y$ and $\delta \phi$ in terms of isospin harmonics. In this way one obtains a Sturm-Liouville problem, the solutions of which reveal the features mentioned in the last paragraph of Section 5.5 [38].

\subsection{The stationary Einstein-Maxwell system}

In the one-dimensional Abelian case, both the off-diagonal Einstein equation (6.17) and the Maxwell equation (6.18) give rise to scalar potentials, (locally) defined by

$$
\mathrm{d} \psi:=V \bar{*}(\bar{F}+\phi f), \quad \mathrm{d} Y:=-V^{2} \bar{*} f+2 \phi \mathrm{d} \psi-2 \psi \mathrm{d} \phi .
$$

Similarly to the vacuum case, this enables one to apply the Lagrange multiplier method to express the effective action in terms of the scalar fields $Y$ and $\psi$, rather than the one-forms $a$ and $\bar{A}$. It turns out that in the stationary-axisymmetric case, to which we return in Section 8, we will also be interested in the dimensional reduction of the EM system with respect to a space-like Killing field. Therefore, we give here the general result for an arbitrary Killing field $\xi$ with norm $N$ :

$$
S_{\text {eff }}=\int \bar{*}\left(\bar{R}-2 \frac{|\mathrm{d} \phi|^{2}+|\mathrm{d} \psi|^{2}}{N}-\frac{|\mathrm{d} N|^{2}+|\mathrm{d} Y-2 \phi \mathrm{d} \psi+2 \psi \mathrm{d} \phi|^{2}}{2 N^{2}}\right),
$$

where $\bar{*}|\mathrm{~d} \phi|^{2}=\mathrm{d} \phi \wedge \bar{*} \mathrm{~d} \phi$, etc. The electro-magnetic potentials $\phi$ and $\psi$ and the gravitational scalars $N$ and $Y$ are obtained from the four-dimensional field strength $F$ and the Killing field as follows (given a two-form $\beta$, we denote by $i_{\xi} \beta$ the one-form with components $\xi^{\mu} \beta_{\mu \nu}$ ):

$$
\begin{gathered}
\mathrm{d} \phi=-i_{\xi} F, \quad \mathrm{~d} \psi=i_{\xi} * F, \\
N=g(\xi, \xi), \quad \mathrm{d} Y=2(\omega+\phi \mathrm{d} \psi-\psi \mathrm{d} \phi),
\end{gathered}
$$

where $2 \omega:=*(\xi \wedge \mathrm{d} \xi)$. The inner product $\langle\cdot, \cdot\rangle$ and the associated "norm" $|\cdot|$ are taken with respect to the three-metric $\overline{\boldsymbol{g}}$, which becomes pseudo-Riemannian if $\xi$ is space-like. The additional stationary symmetry will then imply that the inner products in (6.20) have a fixed sign, despite the fact that $\overline{\boldsymbol{g}}$ is not a Riemannian metric in this case.

The action (6.20) describes a harmonic mapping into a four-dimensional target space, effectively coupled to three-dimensional gravity. In terms of the complex Ernst potentials, $\Lambda:=-\phi+i \psi$ and $\mathrm{E}:=-N-\Lambda \bar{\Lambda}+i Y[102,103]$, the effective EM action becomes

$$
S_{\mathrm{eff}}=\int \bar{*}\left(\bar{R}-2 \frac{|\mathrm{d} \Lambda|^{2}}{N}-\frac{1}{2} \frac{|\mathrm{dE}+2 \bar{\Lambda} \mathrm{d} \Lambda|^{2}}{N^{2}}\right),
$$

where $|\mathrm{d} \Lambda|^{2}:=\langle\mathrm{d} \Lambda, \overline{\mathrm{d}} \Lambda\rangle$. The field equations are obtained from variations with respect to the three-metric $\overline{\boldsymbol{g}}$ and the Ernst potentials. In particular, the equations for $\mathrm{E}$ and $\Lambda$ become

$$
\bar{\Delta} \mathrm{E}=-\frac{\langle\mathrm{dE}, \mathrm{dE}+2 \bar{\Lambda} \mathrm{d} \Lambda\rangle}{N}, \quad \bar{\Delta} \Lambda=-\frac{\langle\mathrm{d} \Lambda, \mathrm{dE}+2 \bar{\Lambda} \mathrm{d} \Lambda\rangle}{N},
$$

where $-N=\Lambda \bar{\Lambda}+\frac{1}{2}(\mathrm{E}+\overline{\mathrm{E}})$. The isometries of the target manifold are obtained by solving the respective Killing equations [250] (see also [186, 187, 189, 188]). This reveals the coset structure 
of the target space and provides a parametrization of the latter in terms of the Ernst potentials. For vacuum gravity and a timelike Killing vector we have seen in Section 6.2 that the coset space, $G / H$, is $S U(1,1) / U(1)$, whereas one finds $G / H=S U(2,1) / S(U(1,1) \times U(1))$ for the stationary $\mathrm{EM}$ equations. If the dimensional reduction is performed with respect to a space-like Killing field, then $G / H=S U(2,1) / S(U(2) \times U(1))$. The explicit representation of the coset manifold in terms of the above Ernst potentials, $\mathrm{E}$ and $\Lambda$, is given by the Hermitian matrix $\Phi$, with components

$$
\Phi_{A B}=\eta_{A B}+2 \operatorname{sig}(N) \bar{v}_{A} v_{B}, \quad\left(v_{0}, v_{1}, v_{2}\right):=\frac{1}{2 \sqrt{|N|}}(\mathrm{E}-1, \mathrm{E}+1,2 \Lambda)
$$

where $v_{A}$ is the Kinnersley vector [185], and $\eta:=\operatorname{diag}(-1,+1,+1)$. It is straightforward to verify that, in terms of $\Phi$, the effective action (6.23) assumes the $S U(2,1)$ invariant form (6.9). The equations of motion following from this action are the following three-dimensional Einstein equations with sources, obtained from variations with respect to $\overline{\boldsymbol{g}}$, and the $\sigma$-model equations, obtained from variations with respect to $\Phi$ :

$$
\bar{R}_{i j}=\frac{1}{4} \operatorname{Trace}\left(\mathcal{J}_{i} \mathcal{J}_{j}\right), \quad \mathrm{d} \overline{\mathcal{J}}=0 ;
$$

here all operations are taken with respect to $\overline{\boldsymbol{g}}$. 


\section{Some Applications}

The $\sigma$-model structure is responsible for various distinguished features of the stationary EM system and related self-gravitating matter models. This section is devoted to a brief discussion of some applications. We show how the Mazur identity [230], the quadratic mass formulae [153] and the Israel-Wilson-Perjés class of stationary black holes [179, 267] arise from the $\sigma$-model structure of the stationary field equations.

\subsection{The Mazur identity}

In the presence of a second Killing field, the EM equations (6.26) experience further, considerable simplifications, which will be discussed later. In this section we will not yet require the existence of an additional Killing symmetry. The Mazur identity [230], which is the key to the uniqueness theorem for the Kerr-Newman metric [228, 229], is a consequence of the coset structure of the field equations. Note, however, that while the derivation of the general form of this identity only requires one Killing vector, its application to the uniqueness argument uses two; we will return to this issue shortly.

In order to obtain the Mazur identity, one considers two arbitrary Hermitian matrices, $\Phi_{1}$ and $\Phi_{2}$. The aim is to compute the Laplacian with respect to a metric $\overline{\boldsymbol{g}}$ (which in the application of interest will be flat) of the relative difference $\Psi$, say, between $\Phi_{2}$ and $\Phi_{1}$,

$$
\Psi:=\Phi_{2} \Phi_{1}^{-1}-\mathbb{1} .
$$

It turns out to be convenient to introduce the current matrices $\mathcal{J}_{1}=\Phi_{1}^{-1} \bar{\nabla} \Phi_{1}$ and $\mathcal{J}_{2}=\Phi_{2}^{-1} \bar{\nabla} \Phi_{2}$, and their difference $\mathcal{J}_{\triangle}=\mathcal{J}_{2}-\mathcal{J}_{1}$, where $\bar{\nabla}$ denotes the covariant derivative with respect to $\overline{\boldsymbol{g}}$. Using $\bar{\nabla} \Psi=\Phi_{2} \mathcal{J}_{\triangle} \Phi_{1}^{-1}$, the Laplacian of $\Psi$ becomes

$$
\bar{\Delta} \Psi=\left\langle\bar{\nabla} \Phi_{2}, \mathcal{J}_{\triangle}\right\rangle \Phi_{1}^{-1}+\Phi_{2}\left\langle\mathcal{J}_{\triangle}, \bar{\nabla} \Phi_{1}^{-1}\right\rangle+\Phi_{2}\left(\bar{\nabla} \mathcal{J}_{\triangle}\right) \Phi_{1}^{-1},
$$

where, as before, the inner product $\langle\cdot, \cdot\rangle$ is taken with respect to the three-metric $\overline{\boldsymbol{g}}$ and also involves a matrix multiplication. For hermitian matrices one has $\bar{\nabla} \Phi_{2}=\mathcal{J}_{2}^{\dagger} \Phi_{2}$ and $\bar{\nabla} \Phi_{1}^{-1}=-\Phi_{1}^{-1} \mathcal{J}_{1}^{\dagger}$, which can be used to combine the trace of the first two terms on the right-hand side of the above expression. One easily finds

$$
\operatorname{Trace}(\bar{\Delta} \Psi)=\operatorname{Trace}\left(\left\langle\Phi_{1}^{-1} \mathcal{J}_{\triangle}^{\dagger}, \Phi_{2} \mathcal{J}_{\triangle}\right\rangle+\Phi_{2}\left(\bar{\nabla} \mathcal{J}_{\triangle}\right) \Phi_{1}^{-1}\right) .
$$

The above expression is an identity for the relative difference of two arbitrary Hermitian matrices, with all operations taken with respect to $\overline{\boldsymbol{g}}$ (recall (6.10)). If the latter are solutions of a non-linear $\sigma$-model with action $\int \operatorname{Trace}(\mathcal{J} \wedge \bar{*} \mathcal{J})$, then their currents are conserved [see Eq. (6.26)], implying that the second term on the right-hand side vanishes. Moreover, if the $\sigma$-model describes a mapping with coset space $S U(p, q) / S(U(p) \times U(q))$, then this is parameterized by positive Hermitian matrices of the form $\Phi=g g^{\dagger}$. (We refer to [196, 95], and [26] for the theory of symmetric spaces.) Hence, the "on-shell" restriction of the Mazur identity to $\sigma$-models with coset $S U(p, q) / S(U(p) \times U(q))$ becomes

$$
\operatorname{Trace}(\bar{\Delta} \Psi)=\operatorname{Trace}\left\langle\mathcal{M}, \mathcal{M}^{\dagger}\right\rangle,
$$

where $\mathcal{M}:=g_{1}^{-1} \mathcal{J}_{\triangle}^{\dagger} g_{2}$.

Of decisive importance to the uniqueness proof for the Kerr-Newman metric is the fact that the right-hand side of the above relation is non-negative. In order to achieve this one needs two Killing fields: The requirement that $\Phi$ be represented in the form $g g^{\dagger}$ forces the reduction of the EM system with respect to a space-like Killing field; otherwise the coset is $S U(2,1) / S(U(1,1) \times U(1))$, which is not of the desired form. As a consequence of the space-like reduction, the three-metric 
$\overline{\boldsymbol{g}}$ is not Riemannian, and the right-hand side of Eq. (7.3) is indefinite, unless the matrix valued one-form $\mathcal{M}$ is space-like. This is the case if there exists a time-like Killing field with $L_{k} \Phi=0$, implying that the currents are orthogonal to $k: \mathcal{J}(k)=i_{k} \Phi^{-1} d \Phi=\Phi^{-1} L_{k} \Phi=0$. The reduction of Eq. (7.3) with respect to the second Killing field and the integration of the resulting expression will be discussed in Section 8.

\subsection{Mass formulae}

The stationary vacuum Einstein equations describe a two-dimensional $\sigma$-model coupled to threedimensional gravity. The target manifold is the pseudo-sphere $S O(2,1) / S O(2) \approx S U(1,1) / U(1)$, which is parameterized in terms of the norm and the twist potential of the Killing field (see Section 6.2). The symmetric structure of the target space persists for the stationary EM system, where the four-dimensional coset, $S U(2,1) / S(U(1,1) \times U(1))$, is represented by a hermitian matrix $\Phi$, comprising the two electro-magnetic scalars, the norm of the Killing field and the generalized twist potential (see Section 6.4).

The coset structure of the stationary field equations is shared by various self-gravitating matter models with massless scalars (moduli) and Abelian vector fields. For scalar mappings into a symmetric target space $\bar{G} / \bar{H}$, say, Breitenlohner et al. [31] have classified the models admitting a symmetry group, which is sufficiently large to comprise all scalar fields arising on the effective level ${ }^{10}$ within one coset space, $G / H$. A prominent example of this kind is the EM-dilaton-axion system, which is relevant to $N=4$ supergravity and to the bosonic sector of four-dimensional heterotic string theory: The pure dilaton-axion system has an $S L(2, \mathbb{R})$ symmetry, which persists in dilaton-axion gravity with an Abelian gauge field [114]. Like the EM system, the model also possesses an $S O(1,2)$ symmetry, arising from the dimensional reduction with respect to the Abelian isometry group generated by the Killing field. However, Gal'tsov and Kechkin [116, 117] have shown that the full symmetry group is larger than $S L(2, \mathbb{R}) \times S O(1,2)$ : The target space for dilatonaxion gravity with a $U(1)$ vector field is the coset $S O(2,3) /(S O(2) \times S O(1,2))$ [113]. Using the fact that $S O(2,3)$ is isomorphic to $S p(4, \mathbb{R})$, Gal'tsov and Kechkin [118] were also able to give a parametrization of the target space in terms of $4 \times 4$ (rather than $5 \times 5$ ) matrices. The relevant coset space was shown to be $\operatorname{Sp}(4, \mathbb{R}) / U(1,1)$; for the generalization to the dilaton-axion system with multiple vector fields we refer to [119, 121].

Common to the black-hole solutions of the above models is the fact that their Komar mass can be expressed in terms of the total charges and the area and surface gravity of the horizon [153]. The reason for this is the following: Like the EM equations (6.26), the stationary field equations consist of the three-dimensional Einstein equations and the $\sigma$-model equations,

$$
\bar{R}_{i j}=\frac{1}{4} \operatorname{Trace}\left(\mathcal{J}_{i} \mathcal{J}_{j}\right), \quad \mathrm{d} \overline{\mathcal{J}}=0 .
$$

The current one-form $\mathcal{J}:=\Phi^{-1} \mathrm{~d} \Phi$ is given in terms of the Hermitian matrix $\Phi$, which comprises all scalar fields arising on the effective level. The $\sigma$-model equations, $\mathrm{d} \overline{\mathcal{J}}=0$, include $\operatorname{dim}(G)$ differential current conservation laws, of which $\operatorname{dim}(H)$ are redundant. Integrating all equations over a space-like hypersurface extending from the horizon to infinity, Stokes' theorem yields a set of relations between the charges and the horizon-values of the scalar potentials. A very familiar relation of this kind is the Smarr formula [296]; see Eq. (7.8) below. The crucial observation is that Stokes' theorem provides $\operatorname{dim}(G)$ independent Smarr relations, rather than only $\operatorname{dim}(G / H)$ ones. (This is due to the fact that all $\sigma$-model currents are algebraically independent, although there are $\operatorname{dim}(H)$ differential identities, which can be derived from the $\operatorname{dim}(G / H)$ field equations.)

\footnotetext{
10 In addition to the actual scalar fields, the effective action comprises two gravitational scalars (the norm and the generalized twist potential) and two scalars for each stationary Abelian vector field (electric and magnetic potentials).
} 
The complete set of Smarr type formulae can be used to get rid of the horizon-values of the scalar potentials. In this way one obtains a relation, which involves only the Komar mass, the charges and the horizon quantities. For the EM-dilaton-axion system one finds, for instance [153],

$$
\left(\frac{1}{4 \pi} \kappa \mathcal{A}\right)^{2}=M^{2}+N^{2}+D^{2}+A^{2}-Q^{2}-P^{2},
$$

where $\kappa$ and $\mathcal{A}$ are the surface gravity and the area of the horizon, and the right-hand side comprises the asymptotic flux integrals, that is, the total mass, the NUT charge, the dilaton and axion charges, and the electric and magnetic charges, respectively. The derivation of Eq. (7.5) is not restricted to static configurations. However, when evaluating the surface terms, one assumes that the horizon is generated by the same Killing field that is also used in the dimensional reduction; the asymptotically time-like Killing field $k$. A generalization of the method to rotating black holes requires the evaluation of the potentials (defined with respect to $k$ ) on a Killing horizon, which is generated by $\ell=k+\Omega_{H} m$, rather than $k$.

A very simple illustration of the idea outlined above is the static, purely electric EM system. In this case, the electrovacuum coset $S U(2,1) / S(U(1,1) \times U(1))$ reduces to $G / H=S U(1,1) / \mathbb{R}$. The matrix $\Phi$ is parameterized in terms of the electric potential $\phi$ and the gravitational potential $V:=-k_{\mu} k^{\mu}$. The $\sigma$-model equations comprise $\operatorname{dim}(G)=3$ differential conservation laws, of which $\operatorname{dim}(H)=1$ is redundant:

$$
\begin{gathered}
\mathrm{d} \bar{*}\left(\frac{\mathrm{d} \phi}{V}\right)=0, \quad \mathrm{~d} \bar{*}\left(\frac{\mathrm{d} V}{V}-2 \phi \frac{\mathrm{d} \phi}{V}\right)=0, \\
\mathrm{~d} \bar{*}\left(\left(V+\phi^{2}\right) \frac{\mathrm{d} \phi}{V}-\phi \frac{\mathrm{d} V}{V}\right)=0 .
\end{gathered}
$$

[It is immediately verified that Eq. (7.7) is indeed a consequence of the Maxwell and Einstein Eqs. (7.6).] Integrating Eqs. (7.6) over a space-like hypersurface and using Stokes' theorem yields

$$
Q=Q_{H}, \quad M=\frac{\kappa}{4 \pi} \mathcal{A}+\phi_{H} Q_{H},
$$

which is the well-known Smarr formula; to establish it, one also uses the fact that the electric potential assumes a constant value $\phi_{H}$ on the horizon. Also, the quantity $Q_{H}$ is defined by the flux integral of $* F$ over the horizon (at time $\Sigma$ ), while the corresponding integral of $* \mathrm{~d} k$ gives $\kappa \mathcal{A} / 4 \pi$ (see [153] for details). In a similar way, Eq. (7.7) provides an additional relation of the Smarr type,

$$
Q=2 \phi_{H} \frac{\kappa}{4 \pi} \mathcal{A}+\phi_{H}^{2} Q_{H},
$$

which can be used to compute the horizon-value of the electric potential, $\phi_{H}$. Using this in the Smarr formula (7.8) gives the desired expression for the total mass, $M^{2}=(\kappa \mathcal{A} / 4 \pi)^{2}+Q^{2}$.

In the "extreme" case, the Bogomol'nyi-Prasad-Sommerfield (BPS) bound [128] for the static EM-dilaton-axion system, $0=M^{2}+D^{2}+A^{2}-Q^{2}-P^{2}$, was previously obtained by constructing null geodesics of the target space [86]. For spherically-symmetric configurations with non-degenerate horizons $(\kappa \neq 0)$, Eq. (7.5) was derived by Breitenlohner et al. [31]. In fact, many of the sphericallysymmetric black-hole solutions with scalar and vector fields [126, 131, 122] are known to fulfill Eq. (7.5), where the left-hand side is expressed in terms of the horizon radius (see [120] and references therein). Using the generalized first law of black-hole thermodynamics, Gibbons et al. [130] obtained Eq. (7.5) for spherically-symmetric solutions with an arbitrary number of vector and moduli fields.

The above derivation of the mass formula (7.5) is neither restricted to spherically-symmetric configurations, nor are the solutions required to be static. The crucial observation is that the coset 
structure gives rise to a set of Smarr formulae, which is sufficiently large to derive the desired relation. Although the result (7.5) was established by using the explicit representations of the EM and EM-dilaton-axion coset spaces [153], similar relations are expected to exist in the general case. More precisely, it should be possible to show that the Hawking temperature of all asymptoticallyflat (or asymptotically NUT) non-rotating black holes with massless scalars and Abelian vector fields is given by

$$
T_{H}=\frac{2}{\mathcal{A}} \sqrt{\sum\left(Q_{S}\right)^{2}-\sum\left(Q_{V}\right)^{2}},
$$

provided that the stationary field equations assume the form (7.4), where $\Phi$ is a map into a symmetric space, $G / H$. Here $Q_{S}$ and $Q_{V}$ denote the charges of the scalars (including the gravitational ones) and the vector fields, respectively.

\subsection{The Israel-Wilson-Perjés class}

A particular class of solutions to the stationary EM equations is obtained by requiring that the Riemannian manifold $(\Sigma, \bar{g})$ is flat [179]. For $\bar{g}_{i j}=\delta_{i j}$, the three-dimensional Einstein equations obtained from variations of the effective action (6.23) with respect to $\overline{\boldsymbol{g}}$ become

$$
4 V \Lambda,_{i} \bar{\Lambda},_{j}=\left(\mathrm{E},,_{i}+2 \bar{\Lambda} \Lambda,_{i}\right)\left(\overline{\mathrm{E}}_{j}+2 \Lambda \bar{\Lambda},,_{j}\right),
$$

where, as we are considering stationary configurations, we use the dimensional reduction with respect to the asymptotically-time-like Killing field $k$ with norm $V=-g(k, k)=-N$. Israel and Wilson [179] have shown that all solutions of this equation fulfill $\Lambda=c_{0}+c_{1} \mathrm{E}$. In fact, it is not hard to verify that this ansatz solves Eq. (7.11), provided that the complex constants $c_{0}$ and $c_{1}$ are subject to $c_{0} \bar{c}_{1}+c_{1} \bar{c}_{0}=-1 / 2$. Using asymptotic flatness, and adopting a gauge where the limits at infinity of the electro-magnetic potentials and the twist potential vanish, one has $\mathrm{E}_{\infty}:=\lim _{r \rightarrow \infty} \mathrm{E}=1$ and $\Lambda_{\infty}:=\lim _{r \rightarrow \infty} \Lambda=0$, and thus

$$
\Lambda=\frac{\mathrm{e}^{i \alpha}}{2}(1-\mathrm{E}), \quad \text { where } \alpha \in \mathbb{R} .
$$

It is crucial that this ansatz solves both the equation for $\mathrm{E}$ and the one for $\Lambda$ : One easily verifies that Eqs. (6.24) reduce to the single equation

$$
\bar{\Delta}(1+\mathrm{E})^{-1}=0
$$

where $\bar{\Delta}$ is the three-dimensional flat Laplacian.

For static, purely electric configurations the twist potential $Y$ and the magnetic potential $\psi$ vanish. The ansatz (7.12), together with the definitions of the Ernst potentials, $\mathrm{E}=V-|\Lambda|^{2}+i Y$ and $\Lambda=-\phi+i \psi($ see Section 6.4$)$, yields

$$
1+\mathrm{E}=2 \sqrt{V}, \text { and } \phi=1-\sqrt{V} .
$$

Since $V_{\infty}=1$, the linear relation between $\phi$ and the gravitational potential $\sqrt{V}$ implies $(\mathrm{d} V)_{\infty}=$ $-(2 \mathrm{~d} \phi)_{\infty}$. By virtue of this, the total mass and the total charge of every asymptotically flat, static, purely electric Israel-Wilson-Perjés solution are equal:

$$
M=-\frac{1}{8 \pi} \int * d k=-\frac{1}{4 \pi} \int * F=Q,
$$

where the integral extends over an asymptotic two-sphere. Note that for purely electric configurations one has $F=k \wedge \mathrm{d} \phi / V$; also, staticity implies $k=-V \mathrm{~d} t$ and thus $d k=-k \wedge \mathrm{d} V / V=-F$. 
The simplest nontrivial solution of the flat Poisson equation (7.13), $\bar{\Delta} V^{-1 / 2}=0$, corresponds to a linear combination of $n$ monopole sources $m_{a}$ located at arbitrary points $\underline{x}_{a}$,

$$
V^{-1 / 2}(\underline{x})=1+\sum_{a=1}^{n} \frac{m_{a}}{\left|\underline{x}-\underline{x}_{a}\right|} .
$$

This is the MP solution [262, 220], with spacetime metric $\mathbf{g}=-V \mathrm{~d} t^{2}+V^{-1} \mathrm{~d} \underline{x}^{2}$ and electric potential $\phi=1-\sqrt{V}$. The MP metric describes a regular black-hole spacetime, where the horizon comprises $n$ disconnected components. Hartle and Hawking [139] have shown that all singularities are "hidden" behind these null surfaces. In Newtonian terms, the configuration corresponds to $n$ arbitrarily-located singularities are "hidden" behind these null surfaces. In Newtonian terms, the configuration corresponds to $n$ arbitrarily-located charged mass points with $\left|q_{a}\right|=\sqrt{G} m_{a}$.

Non-static members of the Israel-Wilson-Perjés class were constructed as well [179, 267]. However, these generalizations of the MP multi-black-hole solutions share certain unpleasant properties with NUT spacetime [252] (see also [32, 237]). In fact, the results of [81] (see [139, 78, 154] for previous results) suggest that - except the MP solutions - all configurations obtained by the Israel-Wilson-Perjés technique either fail to be asymptotically flat or have naked singularities. 


\section{Stationary and Axisymmetric Spacetimes}

The presence of two Killing symmetries yields a considerable simplification of the field equations. In fact, for certain matter models the latter become completely integrable [219], provided that the Killing fields satisfy the orthogonal-integrability conditions. Spacetimes admitting two Killing fields provide the framework for both the theory of colliding gravitational waves and the theory of rotating black holes [56]. Although dealing with different physical subjects, the theories are mathematically closely related. We refer the reader to Chandrasekhar's comparison between corresponding solutions of the Ernst equations [55].

This section reviews the structure of the stationary and axisymmetric field equations. We start by recalling the circularity problem. It is argued that circularity is not a generic property of asymptotically-flat, stationary and axisymmetric spacetimes. However, if the symmetry conditions for the matter fields do imply circularity, then the reduction with respect to the second Killing field simplifies the field equations drastically. The systematic derivation of the Kerr-Newman metric and the proof of its uniqueness provide impressive illustrations of this fact.

\subsection{Integrability properties of Killing fields}

Our aim here is to discuss the circularity problem in some more detail. The task is to use the symmetry properties of the matter model in order to establish the orthogonal-integrability conditions for the Killing fields. The link between the relevant components of the stress-energy tensor and the integrability conditions is provided by a general identity for the derivative of the twist of a Killing field $\xi$, say,

$$
\mathrm{d} \omega_{\xi}=*[\xi \wedge R(\xi)],
$$

and Einstein's equations, implying $\xi \wedge R(\xi)=8 \pi[\xi \wedge T(\xi)]$. This follows from the definition of the twist and the Ricci identity for Killing fields, $\Delta \xi=-2 R(\xi)$, where $R(\xi)$ is the one-form with components $[R(\xi)]_{\mu}:=R_{\mu \nu} \xi^{\nu}$; see, e.g., [151], Chapter 2. For a stationary and axisymmetric spacetime with Killing fields (one-forms) $k$ and $m$, Eq. (8.1) implies

$$
\mathrm{d} g\left(m, \omega_{k}\right)=-8 \pi *[m \wedge k \wedge T(k)]
$$

and similarly for $k \leftrightarrow m$. Eq. (8.2) is an identity up to a term involving the Lie derivative of the twist of the first Killing field with respect to the second one ( $\left.\operatorname{since} \mathrm{d} g\left(m, \omega_{k}\right)=L_{m} \omega_{k}-i_{m} \mathrm{~d} \omega_{k}\right)$. In order to establish $L_{m} \omega_{k}=0$, it is sufficient to show that $k$ and $m$ commute in an asymptoticallyflat spacetime. This was first achieved by Carter [44] and later, under more general conditions, by Szabados [304].

The following is understood to also apply for $k \leftrightarrow m$ : By virtue of Eq. (8.2) - and the fact that the condition $m \wedge k \wedge d k=0$ can be written as $g\left(m, \omega_{k}\right)=0$ - the circularity problem is reduced to the following two tasks:

(i) Show that $\mathrm{d} g\left(m, \omega_{k}\right)=0$ implies $g\left(m, \omega_{k}\right)=0$.

(ii) Establish $m \wedge k \wedge T(k)=0$ from the stationary and axisymmetric matter equations.

(i) Since $g\left(m, \omega_{k}\right)$ is a function, it is locally constant if its derivative vanishes. As $m$ vanishes on the rotation axis, this implies $g\left(m, \omega_{k}\right)=0$ in every connected domain of spacetime intersecting the axis. (At this point it is worthwhile to recall that the corresponding step in the staticity theorem requires more effort: Concluding from $\mathrm{d} \omega_{k}=0$ that $\omega_{k}$ vanishes is more involved, since $\omega_{k}$ is a oneform. However, using the Stokes' theorem to integrate an identity for the twist [152] shows that a strictly stationary - not necessarily simply connected - domain of outer communication must be static if $\omega_{k}$ is closed. While this proves the staticity theorem for vacuum and self-gravitating 
scalar fields [152], it does not solve the electrovacuum case. It should be noted that in the context of the proof of uniqueness the strictly stationary property follows from staticity [72] and not the other way around (compare Figure 3).

(ii) While $m \wedge k \wedge T(k)=0$ follows from the symmetry conditions for electro-magnetic fields [43] and for scalar fields [150], it cannot be established for non-Abelian gauge fields [152]. This implies that the usual foliation of spacetime used to integrate the stationary and axisymmetric Maxwell equations is too restrictive to treat the EYM system. This is seen as follows: In Section 6.3 we have derived the formula (6.17). By virtue of Eq. (6.3) this becomes an expression for the derivative of the twist in terms of the electric Yang-Mills potential $\phi_{k}$ (defined with respect to the stationary Killing field $k$ ) and the magnetic one-form $i_{k} * F=V \bar{*}\left(\bar{F}+\phi_{k} f\right)$ :

$$
\mathrm{d}\left[\omega_{k}+4 \hat{\operatorname{tr}}\left(\phi_{k} i_{k} * F\right)\right]=0,
$$

where $\hat{\operatorname{tr}}()$ is a suitably normalized trace (see Eq. (6.15)). Contracting this relation with the axial Killing field $m$, and using again the fact that the Lie derivative of $\omega_{k}$ with respect to $m$ vanishes, yields immediately

$$
\mathrm{d} g\left(m, \omega_{k}\right)=0 \Longleftrightarrow \hat{\operatorname{tr}}\left(\phi_{k}(* F)(k, m)\right)=0 .
$$

The difference between the Abelian and the non-Abelian case is due to the fact that the Maxwell equations automatically imply that the $(\mathrm{km})$-component of $* F$ vanishes, whereas this does not follow from the Yang-Mills equations. In fact, the Maxwell equation $\mathrm{d} * F=0$ and the symmetry property $L_{k} * F=* L_{k} F=0$ imply the existence of a magnetic potential, $\mathrm{d} \psi=(* F)(k, \cdot)$, thus, $(* F)(k, m)=i_{m} \mathrm{~d} \psi=L_{m} \psi=0$. Moreover, the latter do not imply that the Lie algebra valued scalars $\phi_{k}$ and $(* F)(k, m)$ are orthogonal. Hence, circularity is an intrinsic property of the EM system, whereas it imposes additional requirements on non-Abelian gauge fields.

Both staticity and circularity theorems can be established for scalar fields or, more generally, scalar mappings with arbitrary target manifolds: Consider, for instance, a self-gravitating scalar mapping $\phi:(M, \mathbf{g}) \rightarrow(N, \boldsymbol{G})$ with Lagrangian $L[\phi, \mathrm{d} \phi, \mathbf{g}, \boldsymbol{G}]$. The stress energy tensor is of the form

$$
T=P_{A B} \mathrm{~d} \phi^{A} \otimes \mathrm{d} \phi^{B}+P \mathbf{g},
$$

where the functions $P_{A B}$ and $P$ may depend on $\phi, \mathrm{d} \phi$, the spacetime metric $\mathbf{g}$ and the target metric $G$. If $\phi$ is invariant under the action of a Killing field $\xi$-in the sense that $L_{\xi} \phi^{A}=0$ for each component $\phi^{A}$ of $\phi$ - then the one-form $T(\xi)$ becomes proportional to $\xi: T(\xi)=P \xi$. By virtue of the Killing field identity (8.1), this implies that the twist of $\xi$ is closed. Hence, the staticity and the circularity issue for self-gravitating scalar mappings can be established, under appropriate conditions, as in the vacuum case. From this one concludes that (strictly) stationary non-rotating black-hole configuration of self-gravitating scalar fields are static if $L_{k} \phi^{A}=0$, while stationary and axisymmetric ones are circular if $L_{k} \phi^{A}=L_{m} \phi^{A}=0$.

\subsection{Two-dimensional elliptic equations}

The vacuum and the EM equations in the presence of a Killing symmetry describe harmonic maps into coset manifolds, coupled to three-dimensional gravity (see Section 6). This feature is shared by a variety of other self-gravitating theories with scalar (moduli) and Abelian vector fields (see Section 7.2), for which the field equations assume the form (6.26):

$$
\bar{R}_{i j}=\frac{1}{4} \operatorname{Trace}\left(\mathcal{J}_{i} \mathcal{J}_{j}\right), \quad \mathrm{d} \overline{\mathcal{J}}=0,
$$

The current one-form $\mathcal{J}=\Phi^{-1} \mathrm{~d} \Phi$ is given in terms of the Hermitian matrix $\Phi$, which comprises the norm and the generalized twist potential of the Killing field, the fundamental scalar fields and 
the electric and magnetic potentials arising on the effective level for each Abelian vector field. If the dimensional reduction is performed with respect to the axial Killing field $m=\partial_{\varphi}$ with norm $e^{-2 \lambda}:=g(m, m)$, then $\bar{R}_{i j}$ is the Ricci tensor of the pseudo-Riemannian three-metric $\overline{\boldsymbol{g}}$, defined by

$$
\mathbf{g}=e^{-2 \lambda}(\mathrm{d} \varphi+a)^{2}+e^{2 \lambda} \overline{\boldsymbol{g}} .
$$

In the stationary and axisymmetric case under consideration, there exists, in addition to $m$, an asymptotically-time-like Killing field $k$. Since $k$ and $m$ fulfill the orthogonal-integrability conditions, the spacetime metric can locally be written in a $(2+2)$-block diagonal form. Hence, the circularity property implies that

- $(\Sigma, \overline{\boldsymbol{g}})$ is a static pseudo-Riemannian three-dimensional manifold with metric $\overline{\boldsymbol{g}}=-\rho^{2} \mathrm{~d} t^{2}+\tilde{\boldsymbol{g}}$;

- the connection $a$ is orthogonal to the two-dimensional Riemannian manifold $(\tilde{\Sigma}, \tilde{\boldsymbol{g}})$, that is, $a=a_{t} \mathrm{~d} t$

- the functions $a_{t}$ and $\tilde{g}_{a b}$ do not depend on the coordinates $t$ and $\varphi$.

With respect to the resulting Papapetrou metric [263],

$$
\mathbf{g}=e^{-2 \lambda}\left(\mathrm{d} \varphi+a_{t} \mathrm{~d} t\right)^{2}+e^{2 \lambda}\left(-\rho^{2} \mathrm{~d} t^{2}+\tilde{\boldsymbol{g}}\right),
$$

the field equations (8.6) become a set of partial differential equations on the two-dimensional Riemannian manifold $(\tilde{\Sigma}, \tilde{\boldsymbol{g}})$ :

$$
\begin{gathered}
\Delta_{\tilde{\boldsymbol{g}}} \rho=0 \\
\tilde{R}_{a b}-\frac{1}{\rho} \tilde{\nabla}_{b} \tilde{\nabla}_{a} \rho=\frac{1}{4} \operatorname{Trace}\left(\mathcal{J}_{a} \mathcal{J}_{b}\right), \\
\tilde{\nabla}^{a}\left(\rho J_{a}\right)=0,
\end{gathered}
$$

as is seen from the standard reduction of the Ricci tensor $\bar{R}_{i j}$ with respect to the static three-metric $\overline{\boldsymbol{g}}=-\rho^{2} \mathrm{~d} t^{2}+\tilde{\boldsymbol{g}}$. Further $\mathcal{J}_{t}=0$ and $\bar{*} \mathcal{J}=-\rho \mathrm{d} t \wedge \tilde{*} \mathcal{J}$.

The last simplification of the field equations is obtained by choosing $\rho$ as one of the coordinates on $(\tilde{\Sigma}, \tilde{\boldsymbol{g}})$. Roughly speaking, this follows from the fact that $\rho^{2}:=\mathbf{g}_{t \varphi}^{2}-\mathbf{g}_{t t} \mathbf{g}_{\varphi \varphi}$ is non-negative, that its square root $\rho$ is harmonic (with respect to the Riemannian two-metric $\tilde{\boldsymbol{g}}$ ), and that the domain of outer communications of a stationary black-hole spacetime is simply connected; see [79, 76, 64] for details. The function $\rho$ and the conjugate harmonic function $z$ are called Weyl coordinates. With respect to these, the metric $\tilde{\boldsymbol{g}}$ becomes manifestly conformally flat, and one ends up with the spacetime metric

$$
\mathbf{g}=-\rho^{2} e^{-2 \lambda} \mathrm{d} t^{2}+e^{-2 \lambda}\left(\mathrm{d} \varphi+a_{t} \mathrm{~d} t\right)^{2}+e^{2 \lambda} e^{2 h}\left(\mathrm{~d} \rho^{2}+\mathrm{d} z^{2}\right)
$$

the $\sigma$-model equations

$$
\partial_{\rho}\left(\rho \mathcal{J}_{\rho}\right)+\partial_{z}\left(\rho \mathcal{J}_{z}\right)=0
$$

and the remaining Einstein equations

$$
\partial_{\rho} h=\frac{\rho}{8} \operatorname{Trace}\left(\mathcal{J}_{\rho} \mathcal{J}_{\rho}-\mathcal{J}_{z} \mathcal{J}_{z}\right), \quad \partial_{z} h=\frac{\rho}{4} \operatorname{Trace}\left(\mathcal{J}_{\rho} \mathcal{J}_{z}\right)
$$

for the function $h(\rho, z)$. It is not hard to verify that Eq. (8.13) is the integrability condition for Eqs. (8.14). Since Eq. (8.10) is conformally invariant, the metric function $h(\rho, z)$ does not appear in the $\sigma$-model equation (8.13). Taking into account that $\rho$ is non-negative, the stationary and axisymmetric equations reduce to an elliptic system for a matrix $\Phi$ on a flat half-plane. Once the solution to Eq. (8.13) is known, the remaining metric function $h(\rho, z)$ is obtained from Eqs. (8.14) by quadrature. 


\subsection{The Ernst equations}

The circular $\sigma$-model equations (8.13) for the EM system, with target space $S U(2,1) / S(U(2) \times$ $U(1)$ ), are called Ernst equations. Here, again, we consider the dimensional reduction with respect to the axial Killing field. The fields can be parameterized in terms of the Ernst potentials $\Lambda=$ $-\phi+i \psi$ and $\mathrm{E}=-e^{-2 \lambda}-\Lambda \bar{\Lambda}+i Y$, where the four scalar potentials are obtained from Eqs. (6.21) and (6.22) with $\xi=m$. Instead of writing out the components of Eq. (8.13) in terms of $\Lambda$ and $\mathrm{E}$, it is more convenient to consider Eqs. (6.24), and to reduce them with respect to a static metric $\overline{\boldsymbol{g}}=-\rho^{2} \mathrm{~d} t^{2}+\tilde{\boldsymbol{g}}$ (see Section 8.2). Introducing the complex potentials $\varepsilon$ and $\lambda$ according to

$$
\varepsilon=\frac{1-\mathrm{E}}{1+\mathrm{E}}, \quad \lambda=\frac{2 \Lambda}{1+\mathrm{E}},
$$

one easily finds the two equations

$$
\Delta_{\delta} \zeta+\left\langle\mathrm{d} \zeta, \frac{\mathrm{d} \rho}{\rho}+\frac{2(\bar{\varepsilon} \mathrm{d} \varepsilon+\bar{\lambda} \mathrm{d} \lambda)}{1-|\varepsilon|^{2}-|\lambda|^{2}}\right\rangle=0,
$$

where $\zeta$ stands for either of the complex potentials $\varepsilon$ or $\lambda$. Here we have exploited the conformal invariance of the equations and used both the Laplacian $\Delta_{\delta}$ and the inner product with respect to a flat two-dimensional metric $\delta$. Indeed, consider two black-hole solutions, then each black hole comes with its own metric $\tilde{\boldsymbol{g}}$. However, the equation is conformally covariant, and the $(\rho, z)$ representation of the metric is manifestly conformally flat, with the same domain of coordinates for both black holes. This allows one to view the problem as that of two different Ernst maps defined on the same flat half-plane in $(\rho, z)$-coordinates.

\subsubsection{A derivation of the Kerr-Newman metric}

The Kerr-Newman metric is easily derived within this formalism. For this it is convenient to introduce, first, prolate spheroidal coordinates $x$ and $y$, defined in terms of the Weyl coordinates $\rho$ and $z$ by

$$
\rho^{2}=\mu^{2}\left(x^{2}-1\right)\left(1-y^{2}\right), \quad z=\mu x y,
$$

where $\mu$ is a constant. The domain of outer communications, that is, the upper half-plane $\rho \geq 0$, corresponds to the semi-strip $\mathcal{S}=\{(x, y)|x \geq 1| y \mid, \leq 1\}$. The boundary $\rho=0$ consists of the horizon $(x=0)$ and the northern $(y=1)$ and southern $(y=-1)$ segments of the rotation axis. In terms of $x$ and $y$, the metric $\tilde{\boldsymbol{g}}$ becomes $\left(x^{2}-1\right)^{-1} \mathrm{~d} x^{2}+\left(1-y^{2}\right)^{-1} \mathrm{~d} y^{2}$, up to a conformal factor, which does not enter Eqs. (8.16). The Ernst equations finally assume the form $\left(\varepsilon_{x}:=\partial_{x} \varepsilon\right.$, etc.)

$$
\begin{aligned}
& \left(1-|\varepsilon|^{2}-|\lambda|^{2}\right)\left\{\partial_{x}\left(x^{2}-1\right) \partial_{x}+\partial_{y}\left(1-y^{2}\right) \partial_{y}\right\} \zeta= \\
& \quad-2\left\{\left(x^{2}-1\right)\left(\bar{\varepsilon} \varepsilon_{x}+\bar{\lambda} \lambda_{x}\right) \partial_{x}+\left(1-y^{2}\right)\left(\bar{\varepsilon} \varepsilon_{y}+\bar{\lambda} \lambda_{y}\right) \partial_{y}\right\} \zeta,
\end{aligned}
$$

where $\zeta$ stand for $\varepsilon$ or $\lambda$. A particularly simple solution to those equations is

$$
\varepsilon=p x+i q y, \quad \lambda=\lambda_{0}, \quad \text { where } p^{2}+q^{2}+\lambda_{0}^{2}=1,
$$

with real constants $p, q$ and $\lambda_{0}$. The norm $e^{-2 \lambda}$, the twist potential $Y$ and the electro-magnetic potentials $\phi$ and $\psi$ (all defined with respect to the axial Killing field) are obtained from the above solution by using Eqs. (8.15) and the expressions $e^{-2 \lambda}=-\operatorname{Re}(\mathrm{E})-|\Lambda|^{2}, Y=\operatorname{Im}(\mathrm{E}), \phi=-\operatorname{Re}(\Lambda)$, $\psi=\operatorname{Im}(\Lambda)$. The off-diagonal element of the metric, $a=a_{t} \mathrm{~d} t$, is obtained by integrating the twist expression (6.3), where the twist one-form is given in Eq. (6.22), and the Hodge dual in Eq. (6.3) now refers to the decomposition (8.7) with respect to the axial Killing field. Eventually, the metric function $h$ is obtained from Eqs. (8.14) by quadratures. 
The solution derived so far is the "conjugate" of the Kerr-Newman solution [56]. In order to obtain the Kerr-Newman metric itself, one has to perform a rotation in the $t \varphi$-plane: The spacetime metric is invariant under $t \rightarrow \varphi, \varphi \rightarrow-t$, if $e^{-2 \lambda}, a_{t}$ and $e^{2 h}$ are replaced by $\alpha e^{-2 \lambda}$, $\alpha^{-1} a_{t}$ and $\alpha e^{2 h}$, where $\alpha:=a_{t}^{2}-e^{4 \lambda} \rho^{2}$. This additional step in the derivation of the Kerr-Newman metric is necessary because the Ernst potentials were defined with respect to the axial Killing field $\partial_{\varphi}$. If, on the other hand, one uses the stationary Killing field $\partial_{t}$, then the Ernst equations are singular at the boundary of the ergoregion.

In terms of Boyer-Lindquist coordinates,

$$
r=m(1+p x), \quad \cos \vartheta=y,
$$

one eventually finds the Kerr-Newman metric in the familiar form:

$$
\mathbf{g}=-\frac{\Delta}{\Xi}\left[\mathrm{d} t-\alpha \sin ^{2} \vartheta \mathrm{d} \varphi\right]^{2}+\frac{\sin ^{2} \vartheta}{\Xi}\left[\left(r^{2}+\alpha^{2}\right) \mathrm{d} \varphi-\alpha \mathrm{d} t\right]^{2}+\Xi\left[\frac{1}{\Delta} \mathrm{d} r^{2}+\mathrm{d} \vartheta^{2}\right]
$$

where the constant $\alpha$ is defined by $a_{t}:=\alpha \sin ^{2} \vartheta$. The expressions for $\Delta, \Xi$ and the electro-magnetic vector potential $A$ show that the Kerr-Newman solution is characterized by the total mass $M$, the electric charge $Q$, and the angular momentum $J=\alpha M$ :

$$
\begin{gathered}
\Delta=r^{2}-2 M r+\alpha^{2}+Q^{2}, \quad \Xi=r^{2}+\alpha^{2} \cos ^{2} \vartheta . \\
A=\frac{Q}{\Xi} r\left[\mathrm{~d} t-\alpha \sin ^{2} \vartheta \mathrm{d} \varphi\right] .
\end{gathered}
$$

\subsection{The uniqueness theorem for the Kerr-Newman solution}

In order to establish uniqueness of the Kerr-Newman metric among the stationary and axisymmetric black-hole configurations, one has to show that two solutions of the Ernst equations (8.19) are equal if they are subject to black-hole boundary conditions on $\partial \mathcal{S}$, where $\mathcal{S}$ is the half-plane $\mathcal{S}=\{(\rho, z) \mid \rho \geq 0\}$. Carter proved non-existence of linearized vacuum perturbations near Kerr by means of a divergence identity [45], which Robinson generalized to electrovacuum spacetimes [279].

\subsubsection{Divergence identities}

Considering two arbitrary solutions of the Ernst equations, Robinson was able to construct an identity [280], the integration of which proved the uniqueness of the Kerr metric. The complicated nature of the Robinson identity dashed the hope of finding the corresponding electrovacuum identity by trial and error methods (see, e.g., [47]). The problem was eventually solved when Mazur [228, 230] and Bunting [41] independently derived divergence identities useful for the problem at hand. Bunting's approach, applying to a general class of harmonic mappings between Riemannian manifolds, yields an identity, which enables one to establish the uniqueness of a harmonic map if the target manifold has negative curvature. We refer the reader to Sections 3.2.5 and 8.4.2 (see also [49]) for discussions related to Bunting's method.

So, consider two solutions of the Ernst equations associated to, a priori, distinct black-hole spacetimes, each endowed with its own metric. As discussed in Section 8.3, Weyl coordinates and conformal invariance allow us to view the Ernst equations as equations on a flat half-plane; alternatively, they may be seen as equations for an axisymmetric field on three-dimensional flat space. The Mazur identity (7.2) applies to the relative difference $\Psi=\Phi_{2} \Phi_{1}^{-1}-\mathbb{1}$ of the associated Hermitian matrices and implies (see Section 7.1 for details and references)

$$
\operatorname{Trace}\left(\Delta_{\gamma} \Psi\right)=\operatorname{Trace}\left\langle\mathcal{M}, \mathcal{M}^{\dagger}\right\rangle
$$


where $\Delta_{\gamma}$ is the Laplace-Beltrami operator of the flat metric $\gamma=d \rho^{2}+d z^{2}+\rho^{2} d \varphi^{2}$; also recall that $\mathcal{M}=g_{1}^{-1} \mathcal{J}_{\triangle}^{\dagger} g_{2}$, with $\mathcal{J}_{\triangle}^{\dagger}$ the difference between the currents.

The reduction of the EM equations with respect to the axial Killing field yields $\sigma$-model equations with $S U(2,1) / S(U(2) \times U(1))$ target (see Section 6.4), in vacuum reduces to $S U(2) / S(U(1) \times$ $U(1))$ (see Section 6.2). Hence, the above formula applies to both the stationary and axisymmetric vacuum or electrovacuum field equations. Now, relying on axisymmetry once more, we can reduce the previous Mazur identity to an equation on the flat half-plane $(\mathcal{S}, \delta)$; integrating and using Stokes' theorem leads to

$$
\int_{\partial \mathcal{S}} \rho * \operatorname{Trace}(\mathrm{d} \Psi)=\int_{\mathcal{S}} \rho \operatorname{Trace}\left\langle\mathcal{M}, \mathcal{M}^{\dagger}\right\rangle \eta_{\delta}
$$

where the volume form $\eta_{\delta}$ and the Hogde dual $*$ are related to the flat metric $\delta=d \rho^{2}+d z^{2}$.

The uniqueness of the Kerr-Newman metric should follow now from

- the fact that the integrand on the right-hand side is non-negative, and

- the fact that the boundary at infinity on the left-hand side vanishes for two solutions with the same mass, electric charge and angular momentum, and

- the expectation that the integral over the axis and horizons, where the integrand becomes singular, vanishes for black-hole configurations with the same quotient-space structure.

In order to establish that $\rho$ Trace $(\mathrm{d} \Psi)=0$ on the boundary $\partial \mathcal{S}$ of the half-plane, ${ }^{11}$ one needs the asymptotic behavior and the boundary and regularity conditions of all potentials. One expects that $\rho$ Trace $(\mathrm{d} \Psi)$ vanishes on the horizon, the axis and at infinity, provided that the solutions have the same mass, charge and angular momentum, but no complete analysis of this has been presented in the literature; see [318] for some partial results. Fortunately, the supplementary difficulties arising from the need to control the derivatives of the fields disappear when the distance-function approach described in the next Section 8.4.2 is used.

\subsubsection{The distance function argument}

An alternative to the divergence identities above is provided by the observation that the distance $d\left(\phi_{1}, \phi_{2}\right)$ between two harmonic maps $\phi_{a}, a=1,2$, with negatively curved target manifold is subharmonic [182, Lemma 8.7.3 and Corollary 8.6.4] (see also the proof of Lemma 2 in [321] following results in [287]):

$$
\Delta_{\delta} d\left(\phi_{1}, \phi_{2}\right) \geq 0
$$

compare (4.2). Here $d$ is the distance function between points on the target manifold and $\Delta_{\delta}$ the flat Laplacian on $\mathbb{R}^{3}$. It turns out that the Ernst equations for the Einstein-Maxwell equations fall in this category; in the vacuum case this is obvious, as the target space is then the two-dimensional hyperbolic space. This is somewhat less evident for the Einstein-Maxwell Ernst map, and can be checked by a direct calculation, or can be justified by general considerations about symmetric spaces; more precisely this follows from [144, Theorem 3.1] after noting that the target spaces of the maps under consideration are of non-compact type (see also [320]).

Using this observation, the key to uniqueness is provided by the following non-standard version of the maximum principle:

Proposition $8.1[75$, Appendix $\mathrm{C}]$ Let $\mathscr{A}$ denote the $z$-axis in $\mathbb{R}^{3}$, and let $f \in C^{0}\left(\mathbb{R}^{3} \backslash \mathscr{A}\right)$ satisfy

$$
\Delta_{\delta} f \geq 0 \quad \text { in } \mathbb{R}^{3} \backslash \mathscr{A}, \quad \text { in the distributional sense, }
$$

\footnotetext{
11 A workable formula for Trace $\Psi$ is provided by (4.3) (compare [228, eq. 4.14]).
} 


$$
0 \leq f \leq 1, \quad \text { on } \mathbb{R}^{3} \backslash \mathscr{A}
$$

and

$$
\lim _{(x, y, z) \in \mathbb{R}^{3} \backslash \mathscr{A},|(x, y, z)| \rightarrow \infty} f(x, y, z)=0 .
$$

Then

$$
f \equiv 0, \quad \text { on } \mathbb{R}^{3} \backslash \mathscr{A}
$$

Hence, to prove uniqueness it remains to verify that $d\left(\phi_{1}, \phi_{2}\right)$ is bounded on $\mathbb{R}^{3} \backslash \mathscr{A}$, and that

$$
f(x):=\frac{d\left(\phi_{1}(x), \phi_{2}(x)\right)}{\sup _{y} d\left(\phi_{1}(y), \phi_{2}(y)\right)}
$$

goes to zero at infinity. The latter property follows immediately from asymptotic flatness. The main work is thus to prove that $f$ remains bounded near the axis. Here one needs to keep in mind that the $(\rho, z)$ coordinate system is constructed in an implicit way by PDE techniques, and that the whole axis is singular from the PDE point of view because of factors of $\rho$ and $\rho^{-1}$ in the equations. In particular the associated harmonic maps tend to infinity in the target manifold when the axis of rotation $\mathscr{A}$ is approached. So the proof of boundedness of $f$ requires considerable effort, with the first complete analysis for non-degenerate horizons in [76]. The major challenge are points where the axes of rotation meet the horizons. The degenerate horizons, first settled in [79], provide supplementary difficulties. The proof of boundedness of $f$ near degenerate horizons proceeds via Hájíček's Theorem [135] (rediscovered independently by Lewandowski and Pawłowski [215], see also [202]), that the near-horizon geometry of degenerate axisymmetric Killing horizons with spherical cross-sections coincides with that of the Kerr-Newman solutions. 


\section{Acknowledgments}

MH takes pleasure in thanking Othmar Brodbeck, Gary Gibbons, Domenico Giulini, Dieter Maison, Gernot Neugebauer, Norbert Straumann, Michael Volkov and Robert Wald for helpful discussions. PTC acknowledges bibliographical advice from Michael Volkov.

This work was supported by SNSF Grant P-21-41840.94 (MH), by a Polish Ministry of Science and Higher Education grant Nr N N201 372736 grant (PTC), by projects PTDC/MAT/108921/2008 and CERN/FP/116377/2010, and by CAMSDG, through FCT plurianual funding (JLC). 


\section{References}

[1] Aichelburg, P.C. and Bizoń, P., "Magnetically Charged Black Holes and their Stability", Phys. Rev. $D, 48,607-615,(1993)$. [DOI], [gr-qc/9212009]. (Cited on pages 31 and 32.)

[2] Alexakis, S., Ionescu, A.D. and Klainerman, S., "Hawking's local rigidity theorem without analyticity", Geom. Funct. Anal., 20, 845-869, (2010). [DOI], [arXiv:0902.1173 [gr-qc]]. (Cited on pages 20 and 24.)

[3] Alexakis, S., Ionescu, A.D. and Klainerman, S., "Uniqueness of smooth stationary black holes in vacuum: small perturbations of the Kerr spaces", Commun. Math. Phys., 299, 89-127, (2010). [DOI], [arXiv:0904.0982 [gr-qc]]. (Cited on pages 20 and 22.)

[4] Anderson, M.T., "On Stationary Vacuum Solutions to the Einstein Equations", Ann. Henri Poincare, 1, 977-994, (2000). [DOI], [gr-qc/0001091]. (Cited on page 31.)

[5] Anderson, M.T., "On the Structure of Solutions to the Static Vacuum Einstein Equations", Ann. Henri Poincare, 1, 995-1042, (2000). [DOI], [gr-qc/0001018]. (Cited on page 31.)

[6] Andersson, L. and Metzger, J., "The area of horizons and the trapped region", Commun. Math. Phys., 290, 941-972, (2009). [DOI], [0708.4252 [gr-qc]]. (Cited on page 25.)

[7] Ansorg, M. and Pfister, H., "A universal constraint between charge and rotation rate for degenerate black holes surrounded by matter", Class. Quantum Grav., 25, 035009, (2008). [DOI], [0708.4196 [gr-qc]]. (Cited on page 25.)

[8] Baade, W. and Zwicky, F., "Cosmic Rays from Super-Novae", Proc. Natl. Acad. Sci. USA, 20, 254-263, (1934). [DOI]. (Cited on page 7.)

[9] Bardeen, J.M., Carter, B. and Hawking, S.W., "The four laws of black hole mechanics", Commun. Math. Phys., 31, 161-170, (1973). [DOI]. Online version (accessed 23 May 2005): http://projecteuclid.org/euclid.cmp/1103858973. (Cited on pages 7 and 13.)

[10] Bartnik, R., "The existence of maximal hypersurfaces in asymptotically flat space-times", Commun. Math. Phys., 94, 155-175, (1984). [DOI]. (Cited on page 22.)

[11] Bartnik, R., "The spherically symmetric Einstein Yang-Mills equations", in Perjés, Z., ed., Relativity Today, Proceedings of the Third Hungarian Relativity Workshop 1989, Relativity Today, pp. 221240, (Nova Science, Commack, NY, 1991). (Cited on page 32.)

[12] Bartnik, R. and Chruściel, P.T., "Boundary value problems for Dirac-type equations", J. reine angew. Math., 579, 13-73, (2005). [DOI], [math.DG/0307278]. (Cited on page 16.)

[13] Bartnik, R.A., Fisher, M. and Oliynyk, T.A., "Static Spherically Symmetric Solutions of the SO(5) Einstein Yang-Mills Equations", J. Math. Phys., 51, 032504, (2010). [DOI], [arXiv:0907.3975 [gr-qc]]. (Cited on page 33.)

[14] Bartnik, R. and McKinnon, J., "Particlelike Solutions of the Einstein-Yang-Mills Equations", Phys. Rev. Lett., 61, 141-144, (1988). [DOI]. (Cited on pages 7, 34, and 38.)

[15] Baxter, J.E. and Winstanley, E., "On the existence of soliton and hairy black hole solutions of $s u(N)$ Einstein-Yang-Mills theory with a negative cosmological constant", Class. Quantum Grav., 25, 245014, (2008). [DOI], [arXiv:0808.2977 [gr-qc]]. (Cited on page 8.)

[16] Beem, J.K., Ehrlich, P.E. and Easley, K.L., Global Lorentzian Geometry, Monographs and Textbooks in Pure and Applied Mathematics, 202, (Marcel Dekker, New York, 1996), 2nd edition. [Google Books]. (Cited on page 10.) 
[17] Beig, R. and Chruściel, P.T., "Killing vectors in asymptotically flat space-times. I. Asymptotically translational Killing vectors and the rigid positive energy theorem", J. Math. Phys., 37, 1939-1961, (1996). [DOI], [gr-qc/9510015]. (Cited on page 9.)

[18] Beig, R. and Chruściel, P.T., "The Isometry Groups of Asymptotically Flat, Asymptotically Empty Space-Times with Timelike ADM Four-Momentum", Commun. Math. Phys., 188, 585-597, (1997). [DOI], [gr-qc/9610034]. (Cited on pages 17 and 20.)

[19] Beig, R. and Chruściel, P.T., "The asymptotics of stationary electro-vacuum metrics in odd spacetime dimensions", Class. Quantum Grav., 24, 867-874, (2007). [DOI], [gr-qc/0612012]. (Cited on page 17.)

[20] Beig, R., Gibbons, G.W. and Schoen, R.M., "Gravitating opposites attract", Class. Quantum Grav., 26, 225013, (2009). [DOI], [0907.1193 [gr-qc]]. (Cited on pages 17 and 24.)

[21] Beig, R. and Schoen, R.M., "On static $n$-body configurations in relativity", Class. Quantum Grav., 26, 075014, (2009). [DOI], [0811.1727 [gr-qc]]. (Cited on pages 17 and 24.)

[22] Belinskii, V.A. and Zakharov, V.E., "Integration of the Einstein equations by means of the inverse scattering problem technique and construction of exact soliton solutions", Sov. Phys. JETP, 48, 985, (1978). (Cited on pages 18, 19, and 24.)

[23] Belinskii, V.A. and Zakharov, V.E., "Stationary gravitational solitons with axial symmetry", Sov. Phys. JETP, 50, 1, (1979). (Cited on pages 18, 19, and 24.)

[24] Bizoń, P., "Colored black holes", Phys. Rev. Lett., 64, 2844-2847, (1990). [DOI]. (Cited on pages 7, 31, and 38.)

[25] Booth, I. and Fairhurst, S., "Extremality conditions for isolated and dynamical horizons", Phys. Rev. D, 77, 084005, (2008). [DOI], [arXiv:0708.2209]. (Cited on page 25.)

[26] Boothby, W.M., An Introduction to Differentiable Manifolds and Riemannian Geometry, Pure and Applied Mathematics, 63, (Academic Press, New York, 1975). [Google Books]. (Cited on pages 37 and 41.)

[27] Boschung, P., Brodbeck, O., Moser, F., Straumann, N. and Volkov, M.S., "Instability of Gravitating Sphalerons", Phys. Rev. D, 50, 3842-3846, (1994). [DOI], [gr-qc/9402045]. (Cited on page 31.)

[28] Breitenlohner, P., Forgács, P. and Maison, D., "Gravitating monopole solutions", Nucl. Phys. B, 383, 357-376, (1992). [DOI]. (Cited on pages 7, 31, and 32.)

[29] Breitenlohner, P., Forgács, P. and Maison, D., "Static Spherically Symmetric Solutions of the Einstein-Yang-Mills Equations", Commun. Math. Phys., 163, 141-172, (1994). [DOI], [ADS]. (Cited on page 31.)

[30] Breitenlohner, P., Forgács, P. and Maison, D., "Gravitating monopole solutions II", Nucl. Phys. B, 442, 126-156, (1995). [DOI], [gr-qc/9412039]. (Cited on pages 31 and 32.)

[31] Breitenlohner, P., Maison, D. and Gibbons, G.W., "Four-Dimensional Black Holes from KaluzaKlein Theories", Commun. Math. Phys., 120, 295-334, (1988). [DOI]. (Cited on pages 8, 19, 29, 42, and 43.)

[32] Brill, D.R., "Electromagnetic Fields in a Homogeneous, Nonisotropic Universe", Phys. Rev. B, 133, 845-848, (1964). [DOI], [ADS]. (Cited on page 45.)

[33] Brodbeck, O., Gravitierende Eichsolitonen und Schwarze Löcher mit Yang-Mills-Haar für beliebige Eichgruppen, Ph.D. thesis, (Universität Zürich, Zürich, 1995). (Cited on pages 33 and 37.)

[34] Brodbeck, O., "On Symmetric Gauge Fields for Arbitrary Gauge and Symmetry Groups", Helv. Phys. Acta, 69, 321-324, (1996). [gr-qc/9610024]. (Cited on pages 33 and 37.) 
[35] Brodbeck, O. and Heusler, M., "Stationary perturbations and infinitesimal rotations of static Einstein-Yang-Mills configurations with bosonic matter", Phys. Rev. D, 56, 6278-6283, (1997). [DOI], [gr-qc/9706064]. (Cited on pages 8, 34, 35, 38, and 39.)

[36] Brodbeck, O., Heusler, M., Lavrelashvili, G., Straumann, N. and Volkov, M.S., "Stability Analysis of New Solutions of the EYM System with Cosmological Constant", Phys. Rev. D, 54, 7338-7352, (1996). [DOI], [hep-th/9605166]. (Cited on page 8.)

[37] Brodbeck, O., Heusler, M. and Straumann, N., "Pulsation of Spherically Symmetric Systems in General Relativity", Phys. Rev. D, 53, 754-761, (1996). [DOI], [gr-qc/9506027]. (Cited on page 31.)

[38] Brodbeck, O., Heusler, M., Straumann, N. and Volkov, M., "Rotating solitons and non-rotating non-static black holes", Phys. Rev. Lett., 79, 4310-4313, (1997). [DOI], [gr-qc/9707057]. (Cited on pages $8,33,34$, and 39.)

[39] Brodbeck, O. and Straumann, N., "A generalized Birkhoff theorem for the Einstein-Yang-Mills system", J. Math. Phys., 34, 2412-2423, (1993). [DOI], [ADS]. (Cited on pages 33 and 37.)

[40] Brodbeck, O. and Straumann, N., "Instability of Einstein-Yang-Mills solitons for arbitrary gauge groups", Phys. Lett. B, 324, 309-314, (1994). [DOI], [gr-qc/9401019]. (Cited on page 31.)

[41] Bunting, G.L., Proof of the uniqueness conjecture for black holes, Ph.D. thesis, (University of New England, Armidale, NSW, 1983). (Cited on pages 19 and 50.)

[42] Bunting, G.L. and Masood-ul Alam, A.K.M., "Nonexistence of multiple black holes in asymptotically Euclidean static vacuum space-time", Gen. Relativ. Gravit., 19, 147-154, (1987). [DOI], [ADS]. (Cited on page 16.)

[43] Carter, B., "Killing Horizons and Orthogonally Transitive Groups in Space-Time", J. Math. Phys., 10, 70-81, (1969). [DOI]. (Cited on pages 12, 17, 18, and 47.)

[44] Carter, B., "The Commutation Property of a Stationary, Axisymmetric System", Commun. Math. Phys., 17, 233-238, (1970). [DOI]. (Cited on page 46.)

[45] Carter, B., "Axisymmetric Black Hole has only Two Degrees of Freedom", Phys. Rev. Lett., 26, 331-332, (1971). [DOI]. (Cited on pages 18 and 50.)

[46] Carter, B., "Black Hole Equilibrium States", in DeWitt, C. and DeWitt, B.S., eds., Black Holes, Based on lectures given at the 23rd session of the Summer School of Les Houches, 1972, pp. 57-214, (Gordon and Breach, New York, 1973). (Cited on pages 12 and 33.)

[47] Carter, B., "The General Theory of the Mechanical, Electromagnetic and Thermodynamic Properties of Black Holes", in Hawking, S.W. and Israel, W., eds., General Relativity: An Einstein Centenary Survey, pp. 294-369, (Cambridge University Press, Cambridge; New York, 1979). (Cited on pages 18 and 50.)

[48] Carter, B., "Bunting Identity and Mazur Identity for Non-Linear Elliptic Systems Including the Black Hole Equilibrium Problem", Commun. Math. Phys., 99, 563-591, (1985). [DOI]. (Cited on page 19.)

[49] Carter, B., "Bunting Identity and Mazur Identity for Non-Linear Elliptic Systems Including the Black Hole Equilibrium Problem", Commun. Math. Phys., 99, 563-591, (1985). [DOI], [ADS]. (Cited on page 50.)

[50] Carter, B., "Mathematical Foundations of the Theory of Relativistic Stellar and Black Hole Configurations", in Carter, B. and Hartle, J.B., eds., Gravitation in Astrophysics: Cargèse 1986, Proceedings of a NATO Advanced Study Institute on Gravitation in Astrophysics, held July 15-31, 1986 in Cargèse, France, NATO ASI Series B, pp. 63-122, (Plenum Press, New York, 1987). (Cited on pages 8,18 , and 33.) 
[51] Carter, B., "Has the black hole equilibrium problem been solved?", in Piran, T., ed., The Eighth Marcel Grossmann Meeting on Recent Developments in Theoretical and Experimental General Relativity, Gravitation and Relativistic Field Theories, Proceedings of the meeting held at the Hebrew University of Jerusalem, June 22-27, 1997, (World Scientific, Singapore, 1999). [gr-qc/9712038]. (Cited on page 7.)

[52] Celotti, A., Miller, J.C. and Sciama, D.W., "Astrophysical evidence for the existence of black holes", Class. Quantum Grav., 16, A3-A21, (1999). [DOI], [astro-ph/9912186]. (Cited on page 7.)

[53] Chandrasekhar, S., "Highly Collapsed Configurations of Stellar Mass", Mon. Not. R. Astron. Soc., 91, 456-466, (1931). [ADS]. (Cited on page 7.)

[54] Chandrasekhar, S., "The Maximum Mass of Ideal White Dwarfs", Astrophys. J., 74, 81-82, (1931). [DOI]. (Cited on page 7.)

[55] Chandrasekhar, S., "How One May Explore the Physical Content of the General Theory of Relativity", in Caldi, D.G. and Mostow, G.D., eds., Proceedings of the Gibbs Symposium, Yale University, May 15-17, 1989, pp. 227-251, (AMS / AIP, Providence, 1990). [Google Books]. (Cited on page 46.)

[56] Chandrasekhar, S., The Mathematical Theory of Black Holes and of Colliding Plane Waves, Selected Papers, 6, (University of Chicago Press, Chicago; London, 1991). [Google Books]. (Cited on pages 8, 46 , and 50.)

[57] Chruściel, P.T., "On completeness of orbits of Killing vector fields", Class. Quantum Grav., 10, 2091-2101, (1993). [DOI], [gr-qc/9304029]. (Cited on pages 10 and 20.)

[58] Chruściel, P.T., "No-Hair' Theorems: Folklore, Conjectures, Results", in Beem, J.K. and Duggal, K.L., eds., Differential Geometry and Mathematical Physics, AMS-CMS Special Session on Geometric Methods in Mathematical Physics, August 15-19, 1993, Vancouver, British Columbia, Canada, Contemporary Mathematics, 170, pp. 23-49, (AMS, Providence, 1994). [gr-qc/9402032]. (Cited on pages 7 and 10.)

[59] Chruściel, P.T., "Uniqueness of Stationary, Electro-Vacuum Black Holes Revisited", Helv. Phys. Acta, 69, 529-552, (1996). [gr-qc/9610010]. (Cited on pages 7 and 10.)

[60] Chruściel, P.T., "On rigidity of analytic black holes", Commun. Math. Phys., 189, 1-7, (1997). [gr-qc/9610011]. (Cited on page 20.)

[61] Chruściel, P.T., "The classification of static vacuum spacetimes containing an asymptotically flat spacelike hypersurface with compact interior", Class. Quantum Grav., 16, 661-687, (1999). [DOI], [gr-qc/9809088]. (Cited on pages 12 and 17.)

[62] Chruściel, P.T., "Towards the classification of static electrovacuum spacetimes containing an asymptotically flat spacelike hypersurface with compact interior", Class. Quantum Grav., 16, 689-704, (1999). [DOI], [gr-qc/9810022]. (Cited on pages 17 and 22.)

[63] Chruściel, P.T., "Black Holes", in Frauendiener, J. and Friedrich, H., eds., The Conformal Structure of Space-Time: Geometry, Analysis, Numerics, Proceedings of the international workshop, Tübingen, Germany, 2-4 April 2001, Lecture Notes in Physics, 604, pp. 61-102, (Springer, Berlin; New York, 2002). [gr-qc/0201053], [Google Books]. (Cited on page 8.)

[64] Chruściel, P.T., "Mass and angular-momentum inequalities for axi-symmetric initial data sets. I. Positivity of mass", Ann. Phys. (N.Y.), 323, 2566-2590, (2008). [DOI], [0710.3680 [gr-qc]]. (Cited on pages 18 and 48. )

[65] Chruściel, P.T., "On higher dimensional black holes with Abelian isometry group", J. Math. Phys., 50, 052501, (2009). [DOI], [0812.3424 [gr-qc]]. (Cited on pages 7 and 28.) 
[66] Chruściel, P.T., "Elements of causality theory", arXiv, e-print, (2011). [arXiv:1110.6706 [gr-qc]]. (Cited on page 10.)

[67] Chruściel, P.T. and Cortier, J., "Maximal analytic extensions of the Emparan-Reall black ring", $J$. Differ. Geom., 85, 425-459, (2010). [arXiv:0807.2309 [gr-qc]]. (Cited on page 26.)

[68] Chruściel, P.T., Cortier, J. and García-Parrado Gómez-Lobo, A., "On the global structure of the Pomeransky-Senkov black holes", Adv. Theor. Math. Phys., 14, 1779-1856, (2011). [arXiv:0911.0802 [gr-qc]]. (Cited on page 26.)

[69] Chruściel, P.T., Delay, E., Galloway, G.J. and Howard, R., "Regularity of Horizons and the Area Theorem", Ann. Henri Poincare, 2, 109-178, (2001). [DOI], [gr-qc/0001003]. Online version (accessed 03 November 2011):

http://www.phys.univ-tours.fr/ piotr/papers/area. (Cited on pages 7, 11, and 16.)

[70] Chruściel, P.T., Eckstein, M., Nguyen, L. and Szybka, S., "Existence of singularities in two-Kerr black holes", Class. Quantum Grav., 28, 245017, (2011). [DOI], [arXiv:1111.1448 [gr-qc]]. (Cited on pages 18, 19, and 25.)

[71] Chruściel, P.T., Eckstein, M. and Szybka, S., "On smoothness of Black Saturns", J. High Energy Phys., 2011(11), 048, (2011). [DOI], [arXiv:1007.3668 [hep-th]]. (Cited on pages 24 and 26.)

[72] Chruściel, P.T. and Galloway, G.J., "Uniqueness of static black holes without analyticity", Class. Quantum Grav., 27, 152001, (2010). [DOI], [1004.0513 [gr-qc]]. (Cited on pages 17, 18, 24, 26, 29, and 47.)

[73] Chruściel, P.T., Galloway, G. and Solis, D., "Topological censorship for Kaluza-Klein space-times", Ann. Henri Poincare, 10, 893-912, (2009). [DOI], [0808.3233 [gr-qc]]. (Cited on pages 7, 16, 25, and 28.)

[74] Chruściel, P.T. and Kondracki, W., "Some Global Charges in Classical Yang-Mills Theory", Phys. Rev. D, 36, 1874-1881, (1987). [DOI], [ADS]. (Cited on page 31.)

[75] Chruściel, P.T., Li, Y. and Weinstein, G., "Mass and angular-momentum inequalities for axisymmetric initial data sets. II. Angular momentum", Ann. Phys. (N.Y.), 323, 2591-2613, (2008). [DOI], [0712.4064v2 [gr-qc]]. (Cited on pages 19 and 51.)

[76] Chruściel, P.T. and Lopes Costa, J., "On uniqueness of stationary vacuum black holes", Asterisque, 321, 195-265, (2008). [0806.0016 [gr-qc]]. (Cited on pages 7, 11, 16, 17, 18, 20, 28, 48, and 52.)

[77] Chruściel, P.T. and Maerten, D., "Killing vectors in asymptotically flat space-times. II. Asymptotically translational Killing vectors and the rigid positive energy theorem in higher dimensions", $J$. Math. Phys., 47, 022502, (2006). [DOI], [gr-qc/0512042]. (Cited on page 9.)

[78] Chruściel, P.T. and Nadirashvili, N.S., "All Electrovac Majumdar-Papapetrou Space-times with Non-Singular Black Holes", Class. Quantum Grav., 12, L17-L23, (1995). [DOI], [gr-qc/9412044]. (Cited on pages 22 and 45.)

[79] Chruściel, P.T. and Nguyen, L., "A Uniqueness Theorem for Degenerate Kerr-Newman Black Holes", Ann. Henri Poincare, 11, 585-609, (2010). [DOI], [arXiv:1002.1737 [gr-qc]]. (Cited on pages 7, 18, 20, 22, 48, and 52.)

[80] Chruściel, P.T., Reall, H.S. and Tod, K.P., "On Israel-Wilson-Perjés black holes", Class. Quantum Grav., 23, 2519-2540, (2006). [DOI], [gr-qc/0512116]. (Cited on page 18.)

[81] Chruściel, P.T., Reall, H.S. and Tod, P., "On non-existence of static vacuum black holes with degenerate components of the event horizon", Class. Quantum Grav., 23, 549-554, (2006). [DOI], [gr-qc/0512041]. (Cited on pages 17, 22, and 45.) 
[82] Chruściel, P.T. and Szybka, S.J., "Stable causality of the Pomeransky-Senkov black holes", Adv. Theor. Math. Phys., 15, 175-178, (2010). [arXiv:1010.0213 [hep-th]]. (Cited on page 26.)

[83] Chruściel, P.T. and Tod, P., "The Classification of Static Electro-Vacuum Space-Times Containing an Asymptotically Flat Spacelike Hypersurface with Compact Interior", Commun. Math. Phys., 271, 577-589, (2007). [DOI], [gr-qc/0512043]. (Cited on page 17.)

[84] Chruściel, P.T. and Wald, R.M., "Maximal Hypersurfaces in Stationary Asymptotically Flat Spacetimes", Commun. Math. Phys., 163, 561-604, (1994). [gr-qc/9304009]. (Cited on pages 20, 26, and 33.)

[85] Chruściel, P.T. and Wald, R.M., "On the Topology of Stationary Black Holes", Class. Quantum Grav., 11, L147-L152, (1994). [DOI], [gr-qc/9410004]. (Cited on pages 7 and 16.)

[86] Clément, G. and Gal'tsov, D.V., "Stationary BPS Solutions to Dilaton-Axion Gravity", Phys. Rev. $D, \mathbf{5 4}, 6136-6152$, (1996). [DOI], [hep-th/9607043]. (Cited on pages 8 and 43.)

[87] Coleman, S., "The Uses of Instantons", in Zichichi, A., ed., The Whys of SubNuclear Physics, Proceedings of the International School of Subnuclear Physics, Erice, Trapani, Sicily, July 23 - August 10, 1977, The Subnuclear Series, 15, pp. 805-916, (Plenum Press, New York, 1979). (Cited on page 31.)

[88] Dain, S. and Reiris, M., "Area-Angular momentum inequality for axisymmetric black holes", Phys. Rev. Lett., 107, 051101, (2011). [DOI], [arXiv:1102.5215 [gr-qc]]. (Cited on page 25.)

[89] de Felice, F. and Clarke, C.J.S., Relativity on Curved Manifolds, Cambridge Monographs on Mathematical Physics, (Cambridge University Press, Cambridge; New York, 1990). (Cited on page 12.)

[90] Deser, S., "Absence of Static Solutions in Source-free Yang-Mills Theory", Phys. Lett. B, 64, 463-465, (1976). [DOI], [ADS]. (Cited on page 31.)

[91] Deser, S., "Absence of Static Einstein-Yang-Mills Excitations in Three Dimensions", Class. Quantum Grav., 1, L1-L2, (1984). [DOI]. (Cited on page 32.)

[92] Dias, O.J.C., Figueras, P., Monteiro, R., Reall, H.S. and Santos, J.E., "An instability of higher-dimensional rotating black holes", J. High Energy Phys., 2010(05), 076, (2010). [DOI], [arXiv:1001.4527 [hep-th]]. (Cited on page 27.)

[93] Dias, O.J.C., Horowitz, G.T. and Santos, J.E., "Black holes with only one Killing field", J. High Energy Phys., 2011(7), 115, (2011). [DOI], [arXiv:1105.4167 [hep-th]]. (Cited on page 8.)

[94] Droz, S., Heusler, M. and Straumann, N., "New Black Hole Solutions with Hair", Phys. Lett. B, 268, 371-376, (1991). [DOI]. (Cited on pages 7 and 31.)

[95] Eichenherr, H. and Forger, M., "More about Non-Linear Sigma Models on Symmetric Spaces", Nucl. Phys. B, 164, 528-535, (1980). [DOI], [ADS]. (Cited on page 41.)

[96] Eichmair, M., "The Plateau problem for marginally outer trapped surfaces", J. Differ. Geom., 83, 551-583, (2009). [0711.4139 [math.DG]]. (Cited on page 25.)

[97] Elvang, H. and Figueras, P., "Black Saturn", J. High Energy Phys., 2007(05), 050, (2007). [DOI], [arXiv:hep-th/0701035]. (Cited on pages 24 and 26.)

[98] Emparan, R., Harmark, T., Niarchos, V. and Obers, N.A., "New Horizons for Black Holes and Branes", J. High Energy Phys., 2010(04), 046, (2010). [DOI], [arXiv:0912.2352 [hep-th]]. (Cited on page 27.)

[99] Emparan, R. and Reall, H.S., "A rotating black ring in five dimensions", Phys. Rev. Lett., 88, 101101, (2002). [DOI], [hep-th/0110260]. (Cited on pages 8 and 26.) 
[100] Emparan, R. and Reall, H.S., "Black rings", Class. Quantum Grav., 23, R169-R197, (2006). [DOI], [hep-th/0608012]. (Cited on page 8.)

[101] Emparan, R. and Reall, H.S., "Black Holes in Higher Dimensions", Living Rev. Relativity, 11, lrr2008-6, (2008). [arXiv:0801.3471 [hep-th]]. URL (accessed 03 November 2011): http://www.livingreviews.org/lrr-2008-6. (Cited on pages 8, 26, and 28.)

[102] Ernst, F.J., "New Formulation of the Axially Symmetric Gravitational Field Problem", Phys. Rev., 167, 1175-1178, (1968). [DOI], [ADS]. (Cited on pages 36 and 39.)

[103] Ernst, F.J., "New Formulation of the Axially Symmetric Gravitational Field Problem. II", Phys. Rev., 168, 1415-1417, (1968). [DOI], [ADS]. (Cited on pages 36 and 39.)

[104] Fisher, M. and Oliynyk, T.A., "There are no Magnetically Charged Particle-like Solutions of the Einstein Yang-Mills Equations for Models with an Abelian Residual Group", Commun. Math. Phys., 312, 137-177, (2012). [DOI], [arXiv:1104.0449 [gr-qc]]. (Cited on page 33.)

[105] Forgács, P. and Manton, N.S., "Space-Time Symmetries in Gauge Theories", Commun. Math. Phys. 72, 15-35, (1980). [DOI], [ADS]. (Cited on page 37.)

[106] Friedman, J.L., Schleich, K. and Witt, D.M., "Topological Censorship", Phys. Rev. Lett., 71, 14861489, (1993). [DOI], [ADS], [gr-qc/9305017]. (Cited on page 16.)

[107] Friedrich, H., Rácz, I. and Wald, R.M., "On the rigidity theorem for space-times with a stationary event horizon or a compact Cauchy horizon", Commun. Math. Phys., 204, 691-707, (1999). [DOI], [gr-qc/9811021]. (Cited on pages 20, 22, and 33.)

[108] Galloway, G.J., "On the Topology of Black Holes", Commun. Math. Phys., 151, 53-66, (1993). [DOI], [ADS]. (Cited on page 16.)

[109] Galloway, G.J., "On the topology of the domain of outer communication", Class. Quantum Grav. 12, L99-L101, (1995). [DOI]. (Cited on pages 7 and 16.)

[110] Galloway, G.J., "A 'Finite Infinity' Version of the FSW Topological Censorship", Class. Quantum Grav., 13, 1471-1478, (1996). [DOI]. (Cited on pages 7 and 16.)

[111] Galloway, G.J. and Schoen, R., "A Generalization of Hawking's Black Hole Topology Theorem to Higher Dimensions", Commun. Math. Phys., 266, 571-576, (2006). [DOI], [arXiv:gr-qc/0509107]. (Cited on page 27.)

[112] Galloway, G.J. and Woolgar, E., "The Cosmic Censor forbids Naked Topology", Class. Quantum Grav., 14, L1-L7, (1997). [DOI], [gr-qc/9609007]. (Cited on page 16.)

[113] Gal'tsov, D.V., "Integrable Systems in String Gravity", Phys. Rev. Lett., 74, 2863-2866, (1995). [DOI], [hep-th/9410217]. (Cited on page 42.)

[114] Gal'tsov, D.V., "Geroch-Kinnersley-Chitre Group for Dilaton-Axion Gravity", in Bordag, M., ed., Quantum Field Theory under the Influence of External Conditions, Proceedings of the International Workshop, Leipzig, Germany, 18-22 September 1995, Teubner-Texte zur Physik, 30, (Teubner, Stuttgart and Leipzig, 1996). [hep-th/9606041]. (Cited on page 42.)

[115] Gal'tsov, D.V., "Square of general relativity", in Wiltshire, D.L., ed., Australasian Conference on General Relativity and Gravitation, Proceedings ACGRG1, University of Adelaide, Australia, 12 17 February, 1996, (University of Adelaide, Adelaide, 1996). [ADS], [gr-qc/9608021]. (Cited on page 36.)

[116] Gal'tsov, D.V. and Kechkin, O.V., "Ehlers-Harrison-Type Transformations in Dilaton-Axion Gravity", Phys. Rev. D, 50, 7394-7399, (1994). [DOI], [hep-th/9407155]. (Cited on page 42.) 
[117] Gal'tsov, D.V. and Kechkin, O.V., "Matrix Dilaton-Axion for the Heterotic String in three Dimensions", Phys. Lett. B, 361, 52-58, (1995). [DOI], [hep-th/9507164]. (Cited on page 42.)

[118] Gal'tsov, D.V. and Kechkin, O.V., "U-Duality and Simplectic Formulation of Dilaton-Axion Gravity", Phys. Rev. D, 54, 1656-1666, (1996). [DOI], [hep-th/9507005]. (Cited on page 42.)

[119] Gal'tsov, D.V. and Letelier, P.S., "Ehlers-Harrison Transformations and Black Holes in DilatonAxion Gravity with Multiple Vector Fields", Phys. Rev. D, 55, 3580-3592, (1997). [DOI], [grqc/9612007]. (Cited on page 42.)

[120] Gal'tsov, D.V. and Letelier, P.S., "Interpolating Black Holes in Dilaton-Axion Gravity", Class. Quantum Grav., 14, L9-L14, (1997). [DOI], [gr-qc/9608023]. (Cited on pages 8 and 43.)

[121] Gal'tsov, D.V. and Sharakin, S.A., "Matrix Ernst Potentials for Einstein-Maxwell-Dilaton-Axion with Multiple Vector Fields", Phys. Lett. B, 399, 250-257, (1997). [DOI], [hep-th/9702039]. (Cited on page 42.)

[122] Garfinkle, D., Horowitz, G.T. and Strominger, A., "Charged black holes in string theory", Phys. Rev. D, 43, 3140-3143, (1991). [DOI], [ADS]. (Cited on pages 29 and 43.)

[123] Genzel, R., Eisenhauer, F. and Gillessen, S., "The Galactic Center Massive Black Hole and Nuclear Star Cluster", Rev. Mod. Phys., 82, 3121-3195, (2010). [DOI], [arXiv:1006.0064 [astro-ph.GA]]. (Cited on page 7.)

[124] Geroch, R., "A Method for Generating Solutions of Einstein's Equations", J. Math. Phys., 12, 918-924, (1971). [DOI]. (Cited on page 35.)

[125] Geroch, R., "A Method for Generating New Solutions of Einstein's Equation. II", J. Math. Phys., 13, 394-404, (1972). [DOI]. (Cited on page 35.)

[126] Gibbons, G.W., "Antigravitating Black Hole Solitons with Scalar Hair in $N=4$ Supergravity", Nucl. Phys. B, 207, 337-349, (1982). [DOI]. (Cited on pages 7, 29, 34, and 43.)

[127] Gibbons, G.W., "Self-gravitating Magnetic Monopoles, Global Monopoles and Black Holes", in Barrow, J.D., Henriques, A.B., Lago, M.T.V.T. and Longair, M.S., eds., The Physical Universe: The Interface Between Cosmology, Astrophysics and Particle Physics, Proceedings of the XII Autumn School of Physics, Lisbon, 1 - 5 October 1990, Lecture Notes in Physics, 383, pp. 110-133, (Springer, Berlin; New York, 1990). [DOI]. (Cited on page 31.)

[128] Gibbons, G.W. and Hull, C.M., "A Bogomolny Bound for General Relativity and Solitons in $N=2$ Supergravity", Phys. Lett. B, 109, 190-194, (1982). [DOI]. (Cited on page 43.)

[129] Gibbons, G.W., Ida, D. and Shiromizu, T., "Uniqueness of (dilatonic) charged black holes and black p-branes in higher dimensions", Phys. Rev. D, 66, 044010, (2002). [DOI], [arXiv:hep-th/0206136]. (Cited on page 29.)

[130] Gibbons, G.W., Kallosh, R.E. and Kol, B., "Moduli, Scalar Charges, and the First Law of Black Hole Thermodynamics", Phys. Rev. Lett., 77, 4992-4995, (1996). [DOI], [hep-th/9607108]. (Cited on page 43.)

[131] Gibbons, G.W. and Maeda, K., "Black holes and membranes in higher-dimensional theories with dilaton fields", Nucl. Phys. B, 298, 741-775, (1988). [DOI]. (Cited on pages 29, 34, and 43.)

[132] Greene, B.R., Mathur, S.D. and O'Neill, C.M., "Eluding the No-Hair Conjecture: Black Holes in Spontaneously Broken Gauge Theories", Phys. Rev. D, 47, 2242-2259, (1993). [DOI], [hepth/9211007]. (Cited on page 7.)

[133] Gubser, S.S., "On non-uniform black branes", Class. Quantum Grav., 19, 4825-4844, (2002). [DOI], [hep-th/0110193]. (Cited on page 27.) 
[134] Hájíček, P., "General Theory of Vacuum Ergospheres", Phys. Rev. D, 7, 2311-2316, (1973). (Cited on page 20.)

[135] Hájíček, P., "Three remarks on axisymmetric stationary horizons", Commun. Math. Phys., 36, 305320, (1974). [DOI], [ADS]. (Cited on page 52.)

[136] Hájíček, P., "Stationary Electrovac Space-times with Bifurcate Horizon", J. Math. Phys., 16, 518527, (1975). (Cited on page 20.)

[137] Harmark, T., "Stationary and axisymmetric solutions of higher-dimensional general relativity", Phys. Rev. D, 70, 124002, (2004). [DOI], [arXiv:hep-th/0408141]. (Cited on page 28.)

[138] Harnad, J., Shnider, S. and Vinet, L., "Group Actions on Principal Bundles and Invariance Conditions for Gauge Fields", J. Math. Phys., 21, 2719-2724, (1980). [DOI], [ADS]. (Cited on pages 33 and 37.)

[139] Hartle, J.B. and Hawking, S.W., "Solutions of the Einstein-Maxwell equations with many black holes", Commun. Math. Phys., 26, 87-101, (1972). [DOI]. (Cited on page 45.)

[140] Hartmann, B., Kleihaus, B. and Kunz, J., "Axially symmetric monopoles and black holes in EinsteinYang-Mills-Higgs theory", Phys. Rev. D, 65, 024027, (2001). [DOI], [arXiv:hep-th/0108129]. (Cited on page 7.)

[141] Hawking, S.W., "Black holes in general relativity", Commun. Math. Phys., 25, 152-166, (1972). [DOI]. (Cited on pages 16 and 20.)

[142] Hawking, S.W., "Particle creation by black holes", Commun. Math. Phys., 43, 199-220, (1975). [DOI]. (Cited on page 7.)

[143] Hawking, S.W. and Ellis, G.F.R., The Large Scale Structure of Space-Time, Cambridge Monographs on Mathematical Physics, (Cambridge University Press, Cambridge, 1973). [Google Books]. (Cited on pages $7,8,10,20$, and 33.)

[144] Helgason, S., Differential Geometry, Lie Groups, and Symmetric Spaces, Graduate Studies in Mathematics, 34, (American Mathematical Society, Providence, RI, 2001). [Google Books]. (Cited on page 51.)

[145] Hennig, J., Ansorg, M. and Cederbaum, C., "A universal inequality between the angular momentum and horizon area for axisymmetric and stationary black holes with surrounding matter", Class. Quantum Grav., 25, 162002, (2008). [DOI], [arXiv:0805.4320]. (Cited on page 25.)

[146] Hennig, J., Cederbaum, C. and Ansorg, M., "A universal inequality for axisymmetric and stationary black holes with surrounding matter in the Einstein-Maxwell theory", Commun. Math. Phys., 293, 449-467, (2010). [DOI]. (Cited on page 25.)

[147] Hennig, J. and Neugebauer, G., "Non-existence of stationary two-black-hole configurations: The degenerate case", Gen. Relativ. Gravit., 43, 3139-3162, (2011). [DOI], [arXiv:1103.5248 [gr-qc]]. (Cited on page 25.)

[148] Herdeiro, C.A.R. and Rebelo, C., "On the interaction between two Kerr black holes", J. High Energy Phys., 2008(10), 017, (2008). [DOI], [0808.3941 [gr-qc]]. (Cited on page 18.)

[149] Heusler, M., "Staticity and Uniqueness of Multiple Black Hole Solutions of $\sigma$-Models", Class. Quantum Grav., 10, 791-799, (1993). [DOI]. (Cited on page 33.)

[150] Heusler, M., "The Uniqueness Theorem for Rotating Black Hole Solutions of Self-gravitating Harmonic Mappings", Class. Quantum Grav., 12, 2021-2036, (1995). [DOI], [gr-qc/9503053]. (Cited on pages 34 and 47.) 
[151] Heusler, M., Black Hole Uniqueness Theorems, (Cambridge University Press, Cambridge; New York, 1996). [Google Books]. (Cited on pages 8, 12, 13, 14, 20, 31, and 46.)

[152] Heusler, M., "No-Hair Theorems and Black Holes with Hair", Helv. Phys. Acta, 69, 501-528, (1996). [gr-qc/9610019]. (Cited on pages 31, 33, 46, and 47.)

[153] Heusler, M., "Bogomol'nyi-type Mass Formulas for a Class of Nonrotating Black Holes", Phys. Rev. $D, \mathbf{5 6}, 961-973,(1997)$. [DOI], [gr-qc/9703015]. (Cited on pages 41, 42, 43, and 44.)

[154] Heusler, M., "On the Uniqueness of the Papapetrou-Majumdar metric", Class. Quantum Grav., 14, L129-L134, (1997). [DOI], [gr-qc/9607001]. (Cited on pages 22 and 45.)

[155] Heusler, M., "Uniqueness Theorems for Black Hole Space-Times", in Hehl, F.W., Metzler, R.J.K. and Kiefer, C., eds., Black Holes: Theory and Observations, Proceedings of the 179th W.E. Heraeus Seminar, held at Bad Honnef, Germany, 18-22 August 1997, Lecture Notes in Physics, 514, pp. 157-186, (Springer, Berlin; New York, 1998). [DOI]. (Cited on page 35.)

[156] Heusler, M., Droz, S. and Straumann, N., "Stability Analysis of Self-Gravitating Skyrmions", Phys. Lett. B, 271, 61-67, (1991). [DOI], [ADS]. (Cited on page 31.)

[157] Heusler, M., Droz, S. and Straumann, N., "Linear Stability of Einstein-Skyrme Black Holes", Phys. Lett. B, 285, 21-26, (1992). [DOI], [ADS]. (Cited on page 31.)

[158] Heusler, M. and Straumann, N., "The First Law of Black Hole Physics for a Class of Nonlinear Matter Models", Class. Quantum Grav., 10, 1299-1322, (1993). [DOI]. (Cited on pages 33 and 38.)

[159] Heusler, M. and Straumann, N., "Mass Variation Formulae for Einstein-Yang-Mills-Higgs and Einstein-dilaton Black Holes", Phys. Lett. B, 315, 55-66, (1993). [DOI], [ADS]. (Cited on page 38.)

[160] Heusler, M. and Straumann, N., "Staticity, Circularity, and the First Law of Black Hole Physics", Int. J. Mod. Phys. D, 3, 199-202, (1994). [DOI], [ADS]. (Cited on page 33.)

[161] Heusler, M., Straumann, N. and Zhou, Z.-H., "Self-Gravitating Solutions of the Skyrme Model and their Stability", Helv. Phys. Acta, 66, 614-632, (1993). (Cited on pages 7 and 31.)

[162] Hollands, S., Holland, J. and Ishibashi, A., "Further Restrictions on the Topology of Stationary Black Holes in Five Dimensions", Ann. Henri Poincare, 12, 279-301, (2011). [DOI], [arXiv:1002.0490 [grqc]]. (Cited on page 27.)

[163] Hollands, S. and Ishibashi, A., "On the 'Stationary Implies Axisymmetric' Theorem for Extremal Black Holes in Higher Dimensions", Commun. Math. Phys., 291, 403-441, (2009). [DOI], [arXiv:0809.2659 [gr-qc]]. (Cited on page 27.)

[164] Hollands, S. and Ishibashi, A., "All Vacuum Near Horizon Geometries in D-dimensions with (D - 3) Commuting Rotational Symmetries", Ann. Henri Poincare, 10, 1537-1557, (2010). [DOI], [arXiv:0909.3462 [gr-qc]]. (Cited on page 30.)

[165] Hollands, S., Ishibashi, A. and Wald, R.M., "A Higher Dimensional Stationary Rotating Black Hole Must be Axisymmetric", Commun. Math. Phys., 271, 699-722, (2007). [DOI], [gr-qc/0605106]. (Cited on pages 13 and 27.)

[166] Hollands, S. and Wald, R.M., "Stability of Black Holes and Black Branes", arXiv, e-print, (2012). [arXiv:1201.0463 [gr-qc]]. (Cited on page 27.)

[167] Hollands, S. and Yazadjiev, S., "Uniqueness Theorem for 5-Dimensional Black Holes with Two Axial Killing Fields", Commun. Math. Phys., 283, 749-768, (2008). [DOI], [0707.2775 [gr-qc]]. (Cited on pages 19 and 29.) 
[168] Hollands, S. and Yazadjiev, S., "A uniqueness theorem for five-dimensional Einstein-Maxwell black holes", Class. Quantum Grav., 25, 095010, (2008). [DOI], [arXiv:0711.1722 [gr-qc]]. (Cited on pages 19 and 29.)

[169] Hollands, S. and Yazadjiev, S., "A Uniqueness Theorem for Stationary Kaluza-Klein Black Holes", Commun. Math. Phys., 302, 631-674, (2011). [DOI], [arXiv:0812.3036 [gr-qc]]. (Cited on pages 27, 28, 29, and 30.)

[170] Horowitz, G.T., "The positive energy theorem and its extensions", in Flaherty, F.J., ed., Asymptotic Behavior of Mass and Spacetime Geometry, Proceedings of the conference held at Oregon State University Corvallis, Oregon, USA, October 17-21, 1983, Lecture Notes in Physics, 202, pp. 1-21, (Springer, Berlin; New York, 1984). [DOI]. (Cited on page 31.)

[171] Horowitz, G.T., "Quantum States of Black Holes", in Wald, R.M., ed., Black Holes and Relativistic Stars, Proceedings of the Symposium dedicated to the memory of Subrahmanyan Chandrasekhar, held in Chicago, December 14-15, 1996, pp. 241-266, (University of Chicago Press, Chicago; London, 1998). [gr-qc/9704072]. (Cited on page 7.)

[172] Horowitz, G.T. and Wiseman, T., "General black holes in Kaluza-Klein theory", in Horowitz, G.T., ed., Black Holes in Higher Dimensions, pp. 69-98, (Cambridge University Press, Cambridge; New York, 2012). [arXiv:1107.5563 [gr-qc]]. (Cited on page 26.)

[173] Ida, D., Ishibashi, A. and Shiromizu, T., "Topology and Uniqueness of Higher Dimensional Black Holes", Prog. Theor. Phys. Suppl., 189, 52-92, (2011). [DOI], [arXiv:1105.3491 [hep-th]]. (Cited on page 26.)

[174] Ionescu, A.D. and Klainerman, S., "On the uniqueness of smooth, stationary black holes in vacuum", Invent. Math., 175, 35-102, (2009). [DOI], [0711.0040 [gr-qc]]. (Cited on pages 20 and 24.)

[175] Ionescu, A.D. and Klainerman, S., "Uniqueness Results for Ill-Posed Characteristic Problems in Curved Space-Times", Commun. Math. Phys., 285, 873-900, (2009). [DOI], [arXiv:0711.0042 [grqc]]. (Cited on page 24.)

[176] Israel, W., "Event Horizons in Static Vacuum Space-Times", Phys. Rev., 164, 1776-1779, (1967). [DOI], [ADS]. (Cited on pages 16 and 32.)

[177] Israel, W., "Event Horizons in Static Electrovac Space-Times", Commun. Math. Phys., 8, 245-260, (1968). [DOI], [ADS]. (Cited on pages 16 and 32.)

[178] Israel, W., "Dark stars: the evolution of an idea", in Hawking, S.W. and Israel, W., eds., Three Hundred Years of Gravitation, pp. 199-276, (Cambridge University Press, Cambridge; New York, 1987). (Cited on page 7.)

[179] Israel, W. and Wilson, G.A., "A Class of Stationary Electromagnetic Vacuum Fields", J. Math. Phys., 13, 865-867, (1972). [DOI]. (Cited on pages 18, 41, 44, and 45.)

[180] Jacobson, T. and Venkatarami, S., "Topology of Event Horizons and Topological Censorship", Class. Quantum Grav., 12, 1055-1061, (1995). [DOI], [gr-qc/9410023]. (Cited on page 16.)

[181] Jadczyk, A., "Symmetry of Einstein-Yang-Mills Systems and Dimensional Reduction", J. Geom. Phys., 1, 97-126, (1984). [DOI], [ADS]. (Cited on page 37.)

[182] Jost, J., Riemannian geometry and geometric analysis, (Springer, Berlin, 1998), 2nd edition. (Cited on page 51.)

[183] Kaluza, T., "Zum Unitätsproblem der Physik", Sitzungsber. Preuss. Akad. Wiss., Phys.-Math. Kl., 1921, 966-972, (1921). (Cited on page 26.) 
[184] Kay, B.S. and Wald, R.M., "Theorems on the Uniqueness and Thermal Properties of Stationary, Nonsingular, Quasifree States on Spacetimes with a Bifurcate Killing Horizon", Phys. Rep., 207, 49-136, (1991). [DOI], [ADS]. (Cited on page 13.)

[185] Kinnersley, W., "Generation of Stationary Einstein-Maxwell Fields", J. Math. Phys., 14, 651-653, (1973). [DOI]. (Cited on page 40.)

[186] Kinnersley, W., "Symmetries of the Stationary Einstein-Maxwell Field Equations. I", J. Math. Phys., 18, 1529-1537, (1977). [DOI], [ADS]. (Cited on page 39.)

[187] Kinnersley, W. and Chitre, D.M., "Symmetries of the Stationary Einstein-Maxwell Field Equations. II", J. Math. Phys., 18, 1538-1542, (1977). [DOI], [ADS]. (Cited on page 39.)

[188] Kinnersley, W. and Chitre, D.M., "Symmetries of the Stationary Einstein-Maxwell Equations. IV. Transformations which preserve asymptotic flatness", J. Math. Phys., 19, 2037-2042, (1978). [DOI], [ADS]. (Cited on page 39.)

[189] Kinnersley, W. and Chitre, D.M., "Symmetries of the Stationary Einstein-Maxwell Field Equations. III", J. Math. Phys., 19, 1926-1931, (1978). [DOI], [ADS]. (Cited on page 39.)

[190] Kleihaus, B. and Kunz, J., "Axially Symmetric Multisphalerons in Yang-Mills-Dilaton Theory", Phys. Lett. B, 392, 135-140, (1997). [DOI], [hep-th/9609180]. (Cited on page 34.)

[191] Kleihaus, B. and Kunz, J., "Static Axially Symmetric Solutions of Einstein-Yang-Mills-Dilaton Theory", Phys. Rev. Lett., 78, 2527-2530, (1997). [DOI], [hep-th/9612101]. (Cited on page 34.)

[192] Kleihaus, B. and Kunz, J., "Static Black-Hole Solutions with Axial Symmetry", Phys. Rev. Lett., 79, 1595-1598, (1997). [DOI], [gr-qc/9704060]. (Cited on pages 8 and 34.)

[193] Kleihaus, B. and Kunz, J., "Static axially symmetric Einstein-Yang-Mills-Dilaton solutions: Regular solutions", Phys. Rev. D, 57, 834-856, (1998). [DOI], [gr-qc/9707045]. (Cited on page 34.)

[194] Kleihaus, B. and Kunz, J., "Static Regular and Black Hole Solutions with Axial Symmetry in EYM and EYMD Theory", in Piran, T., ed., The Eighth Marcel Grossmann Meeting on Recent Developments in Theoretical and Experimental General Relativity, Gravitation and Relativistic Field Theories, Proceedings of the meeting held at the Hebrew University of Jerusalem, June 22-27, 1997, pp. 545-547, (World Scientific, Singapore, 1999). [arXiv:gr-qc/9710047]. (Cited on pages 32 and 34.)

[195] Klein, O., "Quantentheorie und fünfdimensionale Relativitätstheorie", Z. Phys., 37, 895-906, (1926). [DOI]. (Cited on page 26.)

[196] Kobayashi, S. and Nomizu, K., Foundations of Differential Geometry, Vol. 2, (John Wiley, New York, 1969). (Cited on pages 37 and 41.)

[197] Kormendy, J. and Gebhardt, K., "Supermassive black holes in Galactic Nuclei", in Wheeler, J.C. and Martel, H., eds., Relativistic Astrophysics, 20th Texas Symposium, Austin, Texas, 10 -15 December 2000, AIP Conference Proceedings, 586, pp. 363-381, (American Institute of Physics, Melville, NY, 2001). [DOI], [astro-ph/0105230]. (Cited on page 7.)

[198] Kramer, D. and Neugebauer, G., "The superposition of two Kerr solutions", Phys. Lett. A, 75, 259-261, (1980). (Cited on pages 18 and 24.)

[199] Kramer, D., Stephani, H., MacCallum, M.A.H. and Herlt, E., Exact Solutions of Einstein's Field Equations, Cambridge Monographs on Mathematical Physics, (Cambridge University Press, Cambridge; New York, 1980). (Cited on page 35.)

[200] Kudoh, H. and Wiseman, T., "Properties of Kaluza-Klein black holes", Prog. Theor. Phys., 111, 475-507, (2004). [DOI], [arXiv:hep-th/0310104]. (Cited on page 26.) 
[201] Kundt, W. and Trümper, M., "Orthogonal Decomposition of Axi-symmetric Stationary Spacetimes", Z. Phys., 192, 419-422, (1966). [DOI]. (Cited on page 18.)

[202] Kunduri, H.K. and Lucietti, J., "A classification of near-horizon geometries of extremal vacuum black holes", J. Math. Phys., 50, 082502, (2009). [DOI], [arXiv:0806.2051 [hep-th]]. (Cited on pages 30 and 52.)

[203] Kunduri, H.K. and Lucietti, J., "Static near-horizon geometries in five dimensions", Class. Quantum Grav., 26, 245010, (2009). [DOI], [arXiv:0907.0410 [hep-th]]. (Cited on page 30.)

[204] Kunduri, H.K., Lucietti, J. and Reall, H.S., "Near-horizon symmetries of extremal black holes", Class. Quantum Grav., 24, 4169-4190, (2007). [DOI], [arXiv:0705.4214 [hep-th]]. (Cited on page 30.)

[205] Künzle, H.P., "SU(n) Einstein-Yang-Mills fields with spherical symmetry", Class. Quantum Grav., 8, 2283-2297, (1991). [DOI]. (Cited on page 33.)

[206] Künzle, H.P., "Analysis of the Static Spherically Symmetric $S U(n)$ Einstein-Yang-Mills Equations", Commun. Math. Phys., 162, 371-397, (1994). [DOI], [ADS]. (Cited on page 33.)

[207] Künzle, H.P., "Einstein-Yang-Mills Fields with Spherical Symmetry", in Beem, J.K. and Duggal, K.L., eds., Differential Geometry and Mathematical Physics, AMS-CMS Special Session on Geometric Methods in Mathematical Physics, August 15-19, 1993, Vancouver, British Columbia, Canada, Contemporary Mathematics, 170, pp. 167-184, (AMS, Providence, 1994). (Cited on pages 33 and 37.)

[208] Künzle, H.P. and Masood-ul Alam, A.K.M., "Spherically Symmetric Static SU(2) Einstein-YangMills Fields", J. Math. Phys., 31, 928-935, (1990). [DOI]. (Cited on pages 7, 31, and 38.)

[209] Künzle, H.P. and Oliynyk, T.A., "Spherical symmetry of generalized EYMH fields", J. Geom. Phys., 56, 1856-1874, (2006). [DOI], [arXiv:0810.3741 [gr-qc]]. (Cited on page 34.)

[210] Laplace, P.-S., Exposition du Système du Monde, (Imprimerie du Cercle-Social, Paris, 1796). [DOI]. Online version (accessed 10 February 2005):

http://visualiseur.bnf.fr/CadresFenetre?Y=Texte\&M=notice\&D=NUMM-88763. （Cited on page 7.)

[211] Larsen, F., "Rotating Kaluza-Klein black holes", Nucl. Phys. B, 575, 211-230, (2000). [DOI], [arXiv:hep-th/9909102]. (Cited on page 26.)

[212] Lavrelashvili, G. and Maison, D., "Regular and Black Hole Solutions of Einstein-Yang-Mills Dilaton Theory", Nucl. Phys. B, 410, 407-422, (1993). [DOI]. (Cited on page 7.)

[213] Lee, K., Nair, V.P. and Weinberg, E.J., "A Classical Instability of Reissner-Nordström Solutions and the Fate of Magnetically Charged Black Holes", Phys. Rev. Lett., 68, 1100-1103, (1992). [DOI], [hep-th/9111045]. (Cited on page 32.)

[214] Lee, K. and Weinberg, E.J., "Nontopological Magnetic Monopoles and New Magnetically Charged Black Holes", Phys. Rev. Lett., 73, 1203-1206, (1994). [DOI], [hep-th/9406021]. (Cited on pages 31 and 32.)

[215] Lewandowski, J. and Pawlowski, T., "Extremal Isolated Horizons: A Local Uniqueness Theorem", Class. Quantum Grav., 20, 587-606, (2003). [DOI], [gr-qc/0208032]. (Cited on page 52.)

[216] Li, Y.Y. and Tian, G., "Regularity of harmonic maps with prescribed singularities", Commun. Math. Phys., 149, 1-30, (1992). [DOI]. (Cited on pages 18 and 22.)

[217] Lopes Costa, J., "On the classification of stationary electro-vacuum black holes", Class. Quantum Grav., 27, 035010, (2010). [DOI], [0912.0834 [gr-qc]]. (Cited on pages 7 and 20.) 
[218] Maison, D., "Ehlers-Harrison-type transformations for Jordan's extended theory of gravitation", Gen. Relativ. Gravit., 10, 717-723, (1979). [DOI]. (Cited on page 29.)

[219] Maison, D., "On the Complete Integrability of the Stationary, Axially Symmetric Einstein Equations", J. Math. Phys., 20, 871-877, (1979). [DOI]. (Cited on page 46.)

[220] Majumdar, S.D., "A Class of Exact Solutions of Einstein's Field Equations", Phys. Rev., 72, 390-398, (1947). [DOI]. (Cited on pages 22 and 45.)

[221] Malec, E., "The Absence of Static, Smooth Solutions in Einstein-Yang-Mills-Klein-Gordon Theory", Acta Phys. Pol. B, 15, 1101-1109, (1984). Online version (accessed 03 November 2011): http://www.actaphys.uj.edu.pl/vol15/t12.htm. (Cited on page 31.)

[222] Manko, V.S., Ruiz, E. and Sanabria-Gómez, J.D., "Extended multi-soliton solutions of the Einstein field equations. II. Two comments on the existence of equilibrium states", Class. Quantum Grav., 17, 3881-3898, (2000). [DOI]. (Cited on page 25.)

[223] Mars, M., "A spacetime characterization of the Kerr metric", Class. Quantum Grav., 16, 2507-2523, (1999). [DOI], [arXiv:gr-qc/9904070]. (Cited on page 22.)

[224] Mars, M. and Simon, W., "On uniqueness of static Einstein-Maxwell-dilaton black holes", Adv. Theor. Math. Phys., 6, 279-305, (2002). [arXiv:gr-qc/0105023]. (Cited on page 29.)

[225] Masood-ul Alam, A.K.M., "Uniqueness proof of static black holes revisited", Class. Quantum Grav., 9, L53-L55, (1992). [DOI]. (Cited on page 17.)

[226] Masood-ul Alam, A.K.M., "Uniqueness of a static charged dilaton black hole", Class. Quantum Grav., 10, 2649-2656, (1993). [DOI]. (Cited on page 29.)

[227] Mavromatos, N.E. and Winstanley, E., "Existence theorems for hairy black holes in $S U(N)$ EinsteinYang-Mills theories", J. Math. Phys., 39, 4849-4873, (1998). [DOI], [arXiv:gr-qc/9712049]. (Cited on page 31.)

[228] Mazur, P.O., "Proof of Uniqueness of the Kerr-Newman Black Hole Solution", J. Math. Phys., 15, 3173-3180, (1982). (Cited on pages 19, 41, 50, and 51.)

[229] Mazur, P.O., "Black Hole Uniqueness from a Hidden Symmetry of Einstein's Gravity", Gen. Relativ. Gravit., 16, 211-215, (1984). [DOI]. (Cited on pages 19 and 41.)

[230] Mazur, P.O., "A Global Identity for Nonlinear Sigma-Models", Phys. Lett. A, 100, 341-344, (1984). [DOI]. (Cited on pages 19, 41, and 50.)

[231] McClintock, J.E., Narayan, R. and Rybicki, G.B., "On the lack of thermal emission from the quiescent black hole XTE J1118+480: Evidence for the event horizon", Astrophys. J., 615, 402-415, (2004). [DOI], [arXiv:astro-ph/0403251]. (Cited on page 7.)

[232] McClintock, J.E. and Remillard, R.A., "Black hole binaries", in Lewin, W.H.G. and van der Klis, M., eds., Compact Stellar X-Ray Sources, Cambridge Astrophysics Series, 39, pp. 157-214, (Cambridge University Press, Cambridge; New York, 2006). [DOI], [arXiv:astro-ph/0306213], [Google Books]. (Cited on page 7.)

[233] Menou, K., Quataert, E. and Narayan, R., "Astrophysical evidence for black hole event horizons", in Dahdich, N. and Narlikar, J., eds., Gravitation and Relativity: At the Turn of the Millennium, Proceedings of the GR-15 Conference, Pune, India, December 16-21, 1997, pp. 43-65, (IUCAA, Pune, 1998). [gr-qc/9803057]. (Cited on page 7.)

[234] Merritt, D. and Ferrarese, L., "Relationship of black holes to bulges", in Knapen, J.H., Beckman, J.E., Shlosman, I. and Mahoney, T.J., eds., The Central Kiloparsec of Starbursts and AGN: the La Palma Connection, Proceedings of a conference held in Los Cancajos, La Palma, Spain, 7-11 May 2001, ASP Conference Series, 249, pp. 335-362, (Astronomical Society of the Pacific, San Francisco, 2001). [ADS], [astro-ph/0107134]. (Cited on page 7.) 
[235] Michell, J., "On the Means of Discovering the Distance, Magnitude, \&c. of the Fixed Stars...", Philos. Trans. R. Soc. London, 74, 35-57, (1784). [DOI]. (Cited on page 7.)

[236] Minguzzi, E. and Sánchez, M., "The causal hierarchy of spacetimes", in Alekseevsky, D.V. and Baum, H., eds., Recent Developments in Pseudo-Riemannian Geometry, ESI Lectures in Mathematics and Physics, pp. 299-358, (EMS Publishing House, Zürich, 2008). [DOI], [Google Books]. (Cited on page 10.)

[237] Misner, C.W., "The Flatter Regions of Newman, Unti, and Tamburino's Generalized Schwarzschild Space", J. Math. Phys., 4, 924-937, (1963). [DOI]. (Cited on page 45.)

[238] Moncrief, V. and Isenberg, J., "Symmetries of cosmological Cauchy horizons", Commun. Math. Phys., 89, 387-413, (1983). [DOI], [ADS]. (Cited on pages 14 and 20.)

[239] Moncrief, V. and Isenberg, J., "Symmetries of Higher Dimensional Black Holes", Class. Quantum Grav., 25, 195015, (2008). [DOI], [arXiv:0805.1451 [gr-qc]]. (Cited on page 27.)

[240] Morisawa, Y. and Ida, D., "A boundary value problem for the five-dimensional stationary rotating black holes", Phys. Rev. D, 69, 124005, (2004). [DOI], [arXiv:gr-qc/0401100]. (Cited on page 29.)

[241] Moss, I.G., Shiiki, N. and Winstanley, E., "Monopole black hole skyrmions", Class. Quantum Grav., 17, 4161-4174, (2000). [DOI], [arXiv:gr-qc/0005007]. (Cited on page 31.)

[242] Müller, A., "Experimental evidence of black holes", in Bonora, L., Iengo, R., Klabucar, D., Pallua, S. and Picek, I., eds., School on Particle Physics, Gravity and Cosmology, Dubrovnik, August $21-$ September 2, 2006, Proceedings of Science, PoS(P2GC)017, (SISSA, Trieste, 2006). [arXiv:astroph/0701228]. URL (accessed 03 November 2011): http://pos.sissa.it/cgi-bin/reader/conf.cgi?confid=34. (Cited on page 7.)

[243] Müller zum Hagen, H., "On the analyticity of stationary vacuum solutions of Einstein's equation", Proc. Cambridge Philos. Soc., 68, 199-201, (1970). [DOI]. (Cited on page 17.)

[244] Müller zum Hagen, H., Robinson, D.C. and Seifert, H.J., "Black Holes in Static Vacuum SpaceTimes", Gen. Relativ. Gravit., 4, 53-78, (1973). [DOI], [ADS]. (Cited on page 16.)

[245] Müller zum Hagen, H., Robinson, D.C. and Seifert, H.J., "Black Holes in Static Electrovac SpaceTimes", Gen. Relativ. Gravit., 5, 61-72, (1974). [DOI]. (Cited on pages 16 and 17.)

[246] Myers, R.C. and Perry, M.J., "Black Holes in Higher Dimensional Space-Times", Ann. Phys. (N.Y.), 172, 304-347, (1986). [DOI]. (Cited on page 26.)

[247] Narayan, R., Garcia, M.R. and McClintock, J.E., "X-ray Novae and the Evidence for Black Hole Event Horizons", in Gurzadyan, V.G., Jantzen, R.T. and Ruffini, R., eds., The Ninth Marcel Grossmann Meeting on recent developments in theoretical and experimental general relativity, gravitation and relativistic field theories, Part A, Proceedings of the MGIX MM meeting held at the University of Rome 'La Sapienza', July 2-8, 2000, pp. 405-425, (World Scientific, Singapore; River Edge, NJ, 2002). [DOI], [astro-ph/0107387]. (Cited on page 7.)

[248] Narayan, R., Yi, I. and Mahadevan, R., "Explaining the spectrum of Sagittarius A* with a model of an accreting black hole", Nature, 374, 623-625, (1995). [DOI]. (Cited on page 7.)

[249] Neugebauer, G. and Hennig, J., "Stationary two-black-hole configurations: A non-existence proof", J. Geom. Phys., 62, 613-630, (2012). [DOI], [arXiv:1105.5830 [gr-qc]]. (Cited on pages 18, 19, 22, and 25.)

[250] Neugebauer, G. and Kramer, D., "Eine Methode zur Konstruktion stationärer Einstein-MaxwellFelder", Ann. Phys. (Leipzig), 479, 62-71, (1969). [DOI]. (Cited on pages 8, 37, and 39.) 
[251] Neugebauer, G. and Meinel, R., "Progress in relativistic gravitational theory using the inverse scattering method", J. Math. Phys., 44, 3407-3429, (2003). [DOI], [gr-qc/0304086]. (Cited on pages 19 and 25.)

[252] Newman, E.T., Tamburino, L.A. and Unti, T., "Empty-Space Generalization of the Schwarzschild Metric", J. Math. Phys., 4, 915-923, (1963). [DOI]. (Cited on page 45.)

[253] Nomizu, K., "On local and global existence of Killing vector fields", Ann. Math., 72, 105-120, (1960). [DOI]. (Cited on pages 20 and 24.)

[254] Oliynyk, T.A., "An existence proof for the gravitating BPS monopole", Ann. Henri Poincare, 7, 199-232, (2006). [DOI], [arXiv:0810.3735 [gr-qc]]. (Cited on pages 7 and 34.)

[255] Oliynyk, T.A. and Künzle, H.P., "On all possible static spherically symmetric EYM solitons and black holes", Class. Quantum Grav., 19, 457-482, (2002). [DOI], [arXiv:gr-qc/0109075]. (Cited on page 33.)

[256] O'Neill, B., Semi-Riemannian Geometry: With Applications to Relativity, Pure and Applied Mathematics, 103, (Academic Press, San Diego; London, 1983). [Google Books]. (Cited on page 10.)

[257] Oppenheimer, J.R. and Snyder, H., "On Continued Gravitational Contraction", Phys. Rev., 56, 455-459, (1939). [DOI], [ADS]. (Cited on page 7.)

[258] Oppenheimer, J.R. and Volkoff, G.M., "On Massive Neutron Cores", Phys. Rev., 55, 374-381, (1939). [DOI]. (Cited on page 7.)

[259] Orlik, P., Seifert Manifolds, Lecture Notes in Mathematics, 291, (Springer, Berlin; New York, 1972). [DOI]. (Cited on page 17.)

[260] Orlik, P. and Raymond, F., "Actions of the Torus on 4-Manifolds. I", Trans. Amer. Math. Soc., 152, 531-559, (1970). (Cited on page 28.)

[261] Orlik, P. and Raymond, F., "Actions of the torus on 4-manifolds - II", Topology, 13, 89-112, (1974). [DOI]. (Cited on page 28.)

[262] Papapetrou, A., "A Static Solution of the Gravitational Field for an Arbitrary Charge-Distribution", Proc. R. Irish Acad. A, 51, 191-204, (1945). (Cited on pages 22 and 45.)

[263] Papapetrou, A., "Eine Rotationssymmetrische Lösung in der Allgemeinen Relativitätstheorie", Ann. Phys. (Leipzig), 447, 309-315, (1953). [DOI], [ADS]. (Cited on page 48.)

[264] Papapetrou, A., "Champs gravitationnels stationnaires à symétrie axiale", Ann. Inst. Henri Poincare A, 4, 83-105, (1966). Online version (accessed 14 May 2012): http://www. numdam.org/item?id=AIHPA_1966_-4_2_83_0. (Cited on page 18.)

[265] Parker, T. and Taubes, C.H., "On Witten's Proof of the Positive Energy Theorem", Commun. Math. Phys., 84, 223-238, (1982). [DOI], [ADS]. (Cited on page 16.)

[266] Penrose, R., Techniques of Differential Topology in Relativity, Regional Conference Series in Applied Mathematics, 7, (SIAM, Philadelphia, 1972). [Google Books]. (Cited on page 10.)

[267] Perjés, Z., "Solutions of the Coupled Einstein-Maxwell Equations Representing the Fields of Spinning Sources", Phys. Rev. Lett., 27, 1668-1670, (1971). [DOI]. (Cited on pages 18, 41, and 45.)

[268] Pomeransky, A.A., "Complete integrability of higher-dimensional Einstein equations with additional symmetry, and rotating black holes", Phys. Rev. D, 73, 044004, (2006). [DOI], [arXiv:hepth/0507250]. (Cited on pages 18, 19, and 25.)

[269] Pomeransky, A.A. and Sen'kov, R.A., "Black ring with two angular momenta", arXiv, e-print, (2006). [arXiv:hep-th/0612005]. (Cited on page 26.) 
[270] Rácz, I. and Wald, R.M., "Global Extensions of Spacetimes Describing Asymptotic Final States of Black Holes", Class. Quantum Grav., 13, 539-552, (1995). [DOI], [gr-qc/9507055]. (Cited on pages 13 and 14.)

[271] Radu, E. and Winstanley, E., "Static axially symmetric solutions of Einstein-Yang-Mills equations with a negative cosmological constant: Black hole solutions", Phys. Rev. D, 70, 084023, (2004). [DOI], [arXiv:hep-th/0407248]. (Cited on page 8.)

[272] Rasheed, D., "The rotating dyonic black holes of Kaluza-Klein theory", Nucl. Phys. B, 454, 379-401, (1995). [DOI], [arXiv:hep-th/9505038]. (Cited on page 26.)

[273] Raymond, F., "Classification of the actions of the circle on 3-manifolds", Trans. Amer. Math. Soc., 131, 51-78, (1968). [DOI]. (Cited on page 17.)

[274] Reall, H.S., "Higher dimensional black holes and supersymmetry", Phys. Rev. D, 68, 024024, (2003). [DOI], [hep-th/0211290]. (Cited on page 27.)

[275] Rees, M.J., "Astrophysical Evidence for Black Holes", in Wald, R.M., ed., Black Holes and Relativistic Stars, Proceedings of the Symposium dedicated to the memory of Subrahmanyan Chandrasekhar, held in Chicago, December 14-15, 1996, pp. 79-101, (University of Chicago Press, Chicago; London, 1998). [ADS], [astro-phys/9701161]. (Cited on page 7.)

[276] Rees, M.J., "Supermassive Black Holes: Their Formation, and Their Prospects as Probes of Relativistic Gravity", in Kaper, L., van den Heuvel, E.P.J. and Woudt, P.A., eds., Black Holes in Binaries and Galactic Nuclei: Diagnostics, Demography and Formation, Proceedings of the ESO Workshop Held at Garching, Germany, in Honour of Riccardo Giacconi, 6-8 September 1999, ESO Astrophysics Symposia, pp. 351-363, (Springer, Berlin; New York, 2001). [DOI], [astro-ph/9912346]. (Cited on page 7.)

[277] Ridgway, S.A. and Weinberg, E.J., "Are All Static Black Hole Solutions Spherically Symmetric?", Gen. Relativ. Gravit., 27, 1017-1021, (1995). [DOI], [gr-qc/9504003]. (Cited on page 32.)

[278] Ridgway, S.A. and Weinberg, E.J., "Static Black Hole Solutions without Rotational Symmetry", Phys. Rev. D, 52, 3440-3456, (1995). [DOI], [gr-qc/9503035]. (Cited on pages 8 and 32.)

[279] Robinson, D.C., "Classification of Black Holes with Electromagnetic Fields", Phys. Rev., 10, 458460, (1974). [DOI], [ADS]. (Cited on pages 16 and 50.)

[280] Robinson, D.C., "Uniqueness of the Kerr Black Hole", Phys. Rev. Lett., 34, 905-906, (1975). [DOI]. (Cited on pages 19 and 50.)

[281] Robinson, D.C., "A Simple Proof of the Generalization of Israel's Theorem", Gen. Relativ. Gravit., 8, 695-698, (1977). [DOI], [ADS]. (Cited on page 16.)

[282] Rogatko, M., "Uniqueness theorem of static degenerate and nondegenerate charged black holes in higher dimensions", Phys. Rev. D, 67, 084025, (2003). [DOI], [hep-th/0302091]. (Cited on page 26.)

[283] Rogatko, M., "Classification of static charged black holes in higher dimensions", Phys. Rev. D, 73, 124027, (2006). [DOI], [arXiv:hep-th/0606116]. (Cited on page 26.)

[284] Ruback, P., "A new uniqueness theorem for charged black holes", Class. Quantum Grav., 5, L155L159, (1988). [DOI], [ADS]. (Cited on page 17.)

[285] Sadeghian, L. and Will, C.M., "Testing the black hole no-hair theorem at the galactic center: perturbing effects of stars in the surrounding cluster", Class. Quantum Grav., 28, (2011). [DOI], [arXiv:1106.5056 [gr-qc]]. (Cited on page 7.)

[286] Sarbach, O. and Winstanley, E., "On the linear stability of solitons and hairy black holes with a negative cosmological constant: The Odd parity sector", Class. Quantum Grav., 18, 2125-2146, (2001). [DOI]. (Cited on page 8.) 
[287] Schoen, R. and Yau, S.T., "Compact Group Actions and the Topology of Manifolds with Non-Positive Curvature", Topology, 18, 361-380, (1979). [DOI]. (Cited on page 51.)

[288] Schoen, R. and Yau, S.-T., "On the Proof of the Positive Mass Conjecture in General Relativity", Commun. Math. Phys., 65, 45-76, (1979). [DOI], [ADS]. (Cited on page 16.)

[289] Schoen, R. and Yau, S.-T., "Proof of the positive mass theorem. II", Commun. Math. Phys., 79, 231-260, (1981). [DOI], [ADS]. (Cited on page 16.)

[290] Schwarzschild, K., "Über das Gravitationsfeld einer Kugel aus inkompressibler Flüssigkeit nach der Einsteinschen Theorie", Sitzungsber. K. Preuss. Akad. Wiss., Phys.-Math. Kl., 1916(III), 424-434, (1916). [physics/9912033]. Online version (accessed 03 November 2011): http://www. archive.org/details/sitzungsberichte1916deutsch. (Cited on page 7.)

[291] Schwarzschild, K., "Über das Gravitationsfeld eines Massenpunktes nach der Einsteinschen Theorie", Sitzungsber. K. Preuss. Akad. Wiss., Phys.-Math. Kl., 1916(VII), 189-196, (1916). [physics/9905030]. Online version (accessed 03 November 2011): http://www.archive.org/details/sitzungsberichte1916deutsch. (Cited on page 7.)

[292] Shaposhnikov, M.E., "Sphalerons and Baryogenesis", Class. Quantum Grav. Suppl., 10, 147-154, (1993). [DOI]. (Cited on page 31.)

[293] Simon, W., "Characterizations of the Kerr metric", Gen. Relativ. Gravit., 16, 465-476, (1984). [DOI]. (Cited on page 22.)

[294] Simon, W., "A Simple Proof of the Generalized Electrostatic Israel Theorem", Gen. Relativ. Gravit., 17, 761-768, (1985). [DOI], [ADS]. (Cited on pages 16 and 29.)

[295] Simon, W., "Radiative Einstein-Maxwell spacetimes and 'no-hair' theorems", Class. Quantum Grav., 9, 241-256, (1992). [DOI]. (Cited on page 17.)

[296] Smarr, L.L., "Mass Formula for Kerr Black Holes", Phys. Rev. Lett., 30, 71-73, (1973). [DOI], [ADS]. (Cited on page 42.)

[297] Smoller, J.A. and Wasserman, A.G., "Existence of Infinitely-Many Smooth, Static, Global Solutions of the Einstein/Yang-Mills Equations", Commun. Math. Phys., 151, 303-325, (1993). [DOI], [ADS]. Online version (accessed 24 May 2005): http://projecteuclid.org/euclid.cmp/1104252139. (Cited on page 31.)

[298] Smoller, J.A., Wasserman, A.G. and Yau, S.-T., "Existence of Black Hole Solutions for the EinsteinYang/Mills Equations", Commun. Math. Phys., 154, 377-401, (1993). [DOI]. Online version (accessed 24 May 2005): http://projecteuclid.org/euclid.cmp/1104252975. (Cited on page 31.)

[299] Smoller, J.A., Wasserman, A.G., Yau, S.-T. and McLeod, J.B., "Smooth static solutions of the Einstein/Yang-Mills equations", Commun. Math. Phys., 143, 115-147, (1991). [DOI], [ADS]. Online version (accessed 24 May 2005): http://projecteuclid.org/euclid.cmp/1104248845. (Cited on page 31.)

[300] Straumann, N., General Relativity and Relativistic Astrophysics, (Springer, Berlin; New York, 1984). [ADS]. (Cited on page 35.)

[301] Straumann, N. and Zhou, Z.-H., "Instability of a colored black hole solution", Phys. Lett. B, 243, 33-35, (1990). [DOI], [ADS]. (Cited on page 31.)

[302] Sudarsky, D. and Wald, R.M., "Extrema of mass, stationarity, and staticity, and solutions to the Einstein-Yang-Mills equations", Phys. Rev. D, 46, 1453-1474, (1992). [DOI]. (Cited on pages 7, 20,33, and 34.) 
[303] Sudarsky, D. and Wald, R.M., "Mass formulas for stationary Einstein-Yang-Mills black holes and a simple proof of two staticity theorems", Phys. Rev. D, 47, R5209-R5213, (1993). [DOI], [grqc/9305023]. (Cited on pages 7, 20,33, and 34.)

[304] Szabados, L.B., "Commutation properties of cyclic and null Killing symmetries", J. Math. Phys., 28, 2688-2691, (1987). [DOI]. (Cited on page 46.)

[305] Szybka, S., "Stable causality of Black Saturns", J. High Energy Phys., 2011(05), 052, (2011). [DOI], [arXiv:1102.3942 [hep-th]]. (Cited on pages 24 and 26.)

[306] Varzugin, G.G., "Equilibrium configuration of black holes and the method of the inverse scattering problem", Theor. Math. Phys., 111, 345-355, (1997). [DOI]. (Cited on pages 19 and 25.)

[307] Varzugin, G.G., "The interaction force between rotating black holes at equilibrium", Theor. Math. Phys., 116, 367-378, (1998). [DOI]. (Cited on pages 19 and 25.)

[308] Vishveshwara, C.V., "Generalization of the 'Schwarzschild Surface' to Arbitrary Static and Stationary Metrics", J. Math. Phys., 9, 1319-1322, (1968). [DOI]. (Cited on pages 12 and 17.)

[309] Volkov, M.S., Brodbeck, O., Lavrelashvili, G. and Straumann, N., "The number of sphaleron instabilities of the Bartnik-McKinnon solitons and non-Abelian black holes", Phys. Lett. B, 349, 438-442, (1995). [DOI], [hep-th/9502045]. (Cited on page 31.)

[310] Volkov, M.S. and Gal'tsov, D.V., "Non-Abelian Einstein-Yang-Mills Black Holes", JETP Lett., 50, 346-350, (1989). (Cited on pages 7, 31, and 38.)

[311] Volkov, M.S. and Gal'tsov, D.V., "Gravitating non-Abelian solitons and black holes with Yang-Mills fields", Phys. Rep., 319, 1-83, (1999). [DOI], [arXiv:hep-th/9810070]. (Cited on page 31.)

[312] Volkov, M.S. and Straumann, N., "Slowly Rotating Non-Abelian Black Holes", Phys. Rev. Lett., 79, 1428-1431, (1997). [DOI], [hep-th/9704026]. (Cited on pages 34 and 38.)

[313] Volkov, M.S., Straumann, N., Lavrelashvili, G., Heusler, M. and Brodbeck, O., "Cosmological analogues of the Bartnik-McKinnon solutions", Phys. Rev. D, 54, 7243-7251, (1996). [DOI], [hepth/9605089]. (Cited on page 8.)

[314] Wald, R.M., General Relativity, (University of Chicago Press, Chicago, 1984). [ADS], [Google Books]. (Cited on pages 8, 13, and 14.)

[315] Wald, R.M., "On the instability of the $n=1$ Einstein-Yang-Mills black holes and mathematically related systems", J. Math. Phys., 33, 248-255, (1992). [DOI], [ADS]. (Cited on page 31.)

[316] Wald, R.M., "Black Holes and Thermodynamics", in Wald, R.M., ed., Black Holes and Relativistic Stars, Proceedings of the Symposium dedicated to the memory of Subrahmanyan Chandrasekhar, held in Chicago, December 14-15, 1996, pp. 155-176, (University of Chicago Press, Chicago; London, 1998). [gr-qc/9702022]. (Cited on page 7.)

[317] Weinberg, E.J., "Magnetically charged black holes with hair", arXiv, e-print, (1995). [arXiv:grqc/9503032]. (Cited on page 32.)

[318] Weinstein, G., "On Rotating Black Holes in Equilibrium in General Relativity", Commun. Pure Appl. Math., 43, 903-948, (1990). [DOI]. (Cited on page 51.)

[319] Weinstein, G., "On the force between rotating coaxial black holes", Trans. Amer. Math. Soc., 343, 899-906, (1994). [DOI]. (Cited on pages 18 and 22.)

[320] Weinstein, G., "On the Dirichlet problem for harmonic maps with prescribed singularities", Duke Math. J., 77, 135-165, (1995). [DOI]. (Cited on page 51.) 
[321] Weinstein, G., "Harmonic maps with prescribed singularities into Hadamard manifolds", Math. Res. Lett., 3, 835-844, (1996). (Cited on page 51.)

[322] Weinstein, G., "N-black hole stationary and axially symmetric solutions of the Einstein/Maxwell equations", Commun. Part. Diff. Eq., 21, 1389-1430, (1996). [DOI]. (Cited on pages 18, 19, and 24.)

[323] Winstanley, E., "Existence of stable hairy black holes in $s u(2)$ Einstein-Yang-Mills theory with a negative cosmological constant", Class. Quantum Grav., 16, 1963-1978, (1999). [DOI], [arXiv:grqc/9812064]. (Cited on page 8.)

[324] Winstanley, E. and Mavromatos, N.E., "Instability of hairy black holes in spontaneously broken Einstein-Yang-Mills-Higgs systems", Phys. Lett. B, 352, 242-246, (1995). [DOI], [hep-th/9503034]. (Cited on page 31.)

[325] Witten, E., "A new proof of the positive energy theorem", Commun. Math. Phys., 80, 381-402, (1981). [DOI]. Online version (accessed 24 May 2005):

http://projecteuclid.org/euclid.cmp/1103919981. (Cited on page 16.)

[326] Wong, W.W.-Y., "A space-time characterization of the Kerr-Newman metric", Ann. Inst. Henri Poincare, 10, 453-484, (2009). [DOI], [arXiv:0807.1904 [gr-qc]]. (Cited on page 20.)

[327] Yu, P., "On Hawking's Local Rigidity Theorems for Charged Black Holes", Ann. Henri Poincare, 11, 1-21, (2010). [DOI], [arXiv:0903.4723 [gr-qc]]. (Cited on page 20.)

[328] Zhou, Z.-H., "Instability of SU(2) Einstein-Yang-Mills Solitons and Non-Abelian Black Holes", Helv. Phys. Acta, 65, 767-819, (1992). [ADS]. (Cited on page 32.)

[329] Zhou, Z.-H. and Straumann, N., "Nonlinear Perturbations of Einstein-Yang-Mills Solitons and NonAbelian Black Holes", Nucl. Phys. B, 360, 180-196, (1991). [DOI], [ADS]. (Cited on pages 31 and 32.) 